\title{
REALIZATIONS OF NON-COMMUTATIVE RATIONAL FUNCTIONS AROUND A MATRIX CENTRE, I: SYNTHESIS, MINIMAL REALIZATIONS AND EVALUATION ON STABLY FINITE ALGEBRAS
}

\author{
MOTKE PORAT AND VICTOR VINNIKOV
}

\begin{abstract}
In this paper we generalize classical results regarding minimal realizations of non-commutative (nc) rational functions using nc FornasiniMarchesini realizations which are centred at an arbitrary matrix point. We prove the existence and uniqueness of a minimal realization for every nc rational function, centred at an arbitrary matrix point in its domain of regularity. Moreover, we show that using this realization we can evaluate the function on all of its domain (of matrices of all sizes) and also w.r.t any stably finite algebra. As a corollary we obtain a new proof of the theorem by Cohn and Amitsur, that equivalence of two rational expressions over matrices implies the expressions are equivalent over all stably finite algebras. Applications to the matrix valued and the symmetric cases are presented as well.
\end{abstract}

\section{Contents}

Introduction

1. Preliminaries

1.1. NC rational functions

1.2. Evaluations over algebras

2. Realizations of NC Rational Expressions 8

2.1. Existence

2.2. Controllability and observability 14

2.3. Minimal realizations 18

2.4. Kalman decomposition 23

2.5. Example 28

2.6. Cohn's theorem 30

2.7. The McMillan degree $\quad 31$

3. Realizations of NC Rational Functions 34

4. Realizations of Matrix Valued NC Rational Functions 37

5. Realizations of Hermitian NC Rational Functions 41

References $\quad 48$

The research of both authors was partially supported by the US-Israel Binational Science Foundation (BSF) Grant No. 2010432, Deutsche Forschungsgemeinschaft (DFG) Grant No. SCHW 1723/1-1, and Israel Science Foundation (ISF) Grant No. 2123/17. 


\section{INTRODUCTION}

Noncommutative (nc, for short) rational functions are a skew field of fractions - more precisely, the universal skew field of fractions - of the ring of nc polynomials, i.e., polynomials in noncommuting indeterminates (the free associative algebra). Essentially, they are obtained by starting with nc polynomials and applying successive arithmetic operations; a considerable amount of technical details is necessary here since in contrast to the commutative case there is no canonical coprime fraction representation for a nc rational function. NC rational functions originated from several sources: the general theory of free rings and of skew fields (see [20, 45, 21, 22, 52, 54, 53], [23, 24, 26, 25] for comprehensive expositions, and $[65,55]$ for good surveys); the theory of rings with rational identities (see [6], also [17] and [66, Chapter 8]); and rational former power series in the theory of formal languages and finite automata (see [51, 72, 73, 30, 31, 32] and [18] for a good survey).

Much like in the case of rational functions of a single variable [13, 50] (and unlike the case of several commuting variables $[35,46]$ ), nc rational functions that are regular at $\underline{0}$ admit a good state space realization theory, see in particular Theorem 1 below. This was first established in the context of finite automata and recognizable power series, and more recently reformulated, with additional details, in the context of transfer functions of multidimensional systems with evolution along the free monoid (see $[12,8,9,10,4,11]$ ). State space realizations of nc rational functions have figured prominently in work on robust control of linear systems subjected to structured possibly time-varying uncertainty (see $[14,15,56]$ ). Another important application of nc rational functions appears in the area of Linear Matrix Inequalities (LMIs, see, e.g., [59, 58, 67]). Most optimization problems of system theory and control are dimensionless in the sense that the natural variables are matrices, and the problem involves nc rational expressions in these matrix variables which have therefore the same form independent of matrix sizes (see [19, 36, 37]). State space realizations are exactly what is needed to convert (numerically unmanageable) rational matrix inequalities into (highly manageable) linear matrix inequalities (see [43]).

Coming from a different direction, the method of state space realizations, also known as the linearization trick, found important recent applications in free probability, see $[16,41,74,75]$. Here it is crucial to evaluate nc rational expressions on a general algebra — which is stably finite in many important cases - rather than on matrices of all sizes. Stably finite algebras appeared in this context in the work of Cohn [26] and they play an important and not surprising role in our analysis.

Here is a full characterization of nc rational functions which are regular at $\underline{0}$ and their (matrix) domains of regularity, in terms of their minimal realizations (for the proofs, see $[8,9,30,31,32,48,49,72,73])$.

Theorem 1. If $\mathfrak{R}$ is a nc rational function of $x_{1}, \ldots, x_{d}$ and $\mathfrak{R}$ is regular at $\underline{0}$, then $\mathfrak{R}$ admits a unique (up to unique similarity) minimal nc Fornasini-Marchesini realization

$$
\mathfrak{R}\left(x_{1}, \ldots, x_{d}\right)=D+C\left(I_{L}-\sum_{k=1}^{d} A_{k} x_{k}\right)^{-1} \sum_{k=1}^{d} B_{k} x_{k},
$$

where $A_{1}, \ldots, A_{d} \in \mathbb{K}^{L \times L}, B_{1}, \ldots, B_{d} \in \mathbb{K}^{L \times 1}, C \in \mathbb{K}^{1 \times L}, D=\mathfrak{R}(\underline{0}) \in \mathbb{K}$ and $L \in \mathbb{N}$. Moreover, for all $m \in \mathbb{N}:\left(X_{1}, \ldots, X_{d}\right) \in\left(\mathbb{K}^{m \times m}\right)^{d}$ is in the domain of 
regularity of $\Re$ if and only if $\operatorname{det}\left(I_{L m}-X_{1} \otimes A_{1}-\ldots-X_{d} \otimes A_{d}\right) \neq 0$; in that case

$$
\mathfrak{R}\left(X_{1}, \ldots, X_{d}\right)=I_{m} \otimes D+\left(I_{m} \otimes C\right)\left(I_{L m}-\sum_{k=1}^{d} X_{k} \otimes A_{k}\right)^{-1} \sum_{k=1}^{d} X_{k} \otimes B_{k} .
$$

Here a realization is called minimal if the state space dimension $L$ is as small as possible; equivalently, the realization is observable, i.e.,

$$
\bigcap_{0 \leq k} \bigcap_{1 \leq i_{1}, \ldots, i_{k} \leq d} \operatorname{ker}\left(C A_{i_{1}} \cdots A_{i_{k}}\right)=\{\underline{0}\}
$$

and controllable, i.e.,

$$
\bigvee_{0 \leq k} \bigvee_{1 \leq i_{1}, \ldots, i_{k}, j \leq d,} A_{i_{1}} \cdots A_{i_{k}} B_{j}=\mathbb{K}^{L}
$$

Theorem 1 is strongly related to expansions of nc rational functions which are regular at $\underline{0}$ into formal nc power series around $\underline{0}$; that is why it is not applicable for all nc rational functions. For example, the nc rational expression $R\left(x_{1}, x_{2}\right)=$ $\left(x_{1} x_{2}-x_{2} x_{1}\right)^{-1}$ is not defined at $\underline{0}$, nor at any pair $\left(y_{1}, y_{2}\right) \in \mathbb{K}^{2}$, therefore one can not consider realizations of $R$ which are centred at $\underline{0}$ as in Theorem 1 , nor at any scalar point (a tuple of scalars). A realization theory for such expressions (and hence functions) is required in particular for all of the applications mentioned above. Such a theory is presented here, using the ideas of the general theory of nc functions.

The theory of $\mathbf{n c}$ functions has its roots in the works by Taylor $[76,77]$ on noncommutative spectral theory. It was further developed by Voiculescu $[80,78,79]$ and Kalyuzhnyi-Verbovetskyi-Vinnikov [47], including a detailed discussion on nc difference-differential calculus. The main underlying idea is that a function of $d$ noncommuting variables is a function of $d$-tuples of square matrices of all sizes that respects direct sums and simultaneous similarities. See also the work of HeltonKlep-McCullough [38, 39], of Popescu [61, 62], of Muhly-Solel [57], and of AglerMcCarthy $[1,2,3]$. A crucial fact [47, Chapters 4-7] is that nc functions admit power series expansions, called Taylor-Taylor series in honor of Brook Taylor and of Joseph L. Taylor, around an arbitrary matrix point in their domain. This motivates us to generalize realizations as in Theorem 1 to the case where the centre is a $d$-tuple of matrices rather than $\underline{0}$ or a $d$-tuple of scalars.

This is the first in a series of papers with the goal of generalizing the theory of (Fornasini-Marchesini) realizations centred at $\underline{0}$ (or at a scalar point), to the case of (Fornasini-Marchesini) realizations centred at an arbitrary matrix point in the domain of regularity of a nc rational function. In particular, we present a generalization of Theorem 1 (see Theorem 2 below) namely the existence and uniqueness of a minimal realization, together with the inclusion of the domain of the nc rational function in the domain of any of its minimal realizations. (The other inclusion and hence the equality of the two domains is presented in a follow-up paper [63])

Other types of realizations of nc rational functions that are not necessary regular at $\underline{0}$ have been considered in $[27,28]$ and in [82], see also the recent papers $[68,69,70,71]$. We will consider further the relation between our representations and those of $[27,28]$ in our follow-up paper [63]. 
Here is an outline of the paper: In Section 1 we give some preliminaries on nc rational functions and evaluations over general algebras.

In Section 2 we present the setting of nc Fornasini-Marchesini realizations centred at a matrix point $\underline{Y} \in\left(\mathbb{K}^{s \times s}\right)^{d}$ and generalize classical results which are well known in the scalar case $(s=1)$ to the case where $s \geq 1$. We prove, using synthesis, the existence of such realizations for any nc rational expression (Theorem 2.4), and introduce the terms of observability and controllability (Subsection 2.2) analogously to the scalar case as in [50]. The uniqueness of minimal realizations, up to unique similarity, is then proved (Theorems 2.13 and 2.16), followed by a Kalman decomposition argument (Theorem 2.15). An example of an explicit construction of a minimal realization is presented in Subsection 2.5 for the nc rational expression $\left(x_{1} x_{2}-x_{2} x_{2}\right)^{-1}$. During the whole section we carry on the results also in a more generalized settings of evaluations w.r.t arbitrary unital stably finite $\mathbb{K}$-algebra; as a corollary we obtain a new proof of a theorem of Cohn that equivalence of two rational expressions over matrices implies their equivalence over all stably finite algebras (Theorem 2.19). Finally, in Subsection 2.7 we define the McMillan degree of a nc rational expression using minimal Fornasini-Marchesini realizations and show that it does not depend on the centre of the realization.

Section 3 contains the main result of the paper, that is a partial generalization of Theorem 1 for nc rational functions not necessary regular at a scalar point:

Theorem 2 (Theorem 3.3, Corollary 2.18). If $\mathfrak{R}$ is a nc rational function of $x_{1}, \ldots, x_{d}$ over $\mathbb{K}$, then for every $\underline{Y}=\left(Y_{1}, \ldots, Y_{d}\right) \in \operatorname{dom}_{s}(\mathfrak{R})$ there exists a unique (up to unique similarity) minimal (observable and controllable) nc Fornasini-Marchesini realization

$$
\mathcal{R}_{\mathcal{F M}}\left(X_{1}, \ldots, X_{d}\right)=D+C\left(I_{L}-\sum_{k=1}^{d} \mathbf{A}_{k}\left(X_{k}-Y_{k}\right)\right)^{-1} \sum_{k=1}^{d} \mathbf{B}_{k}\left(X_{k}-Y_{k}\right)
$$

centred at $\underline{Y}$, such that for every $m \in \mathbb{N}$ and $\left(X_{1}, \ldots, X_{d}\right) \in \operatorname{dom}_{s m}(\mathfrak{R})$ :

$$
\mathfrak{R}\left(X_{1}, \ldots, X_{d}\right)=I_{m} \otimes D+\left(I_{m} \otimes C\right)\left(I_{L m}-\sum_{k=1}^{d}\left(X_{k}-I_{m} \otimes Y_{k}\right) \mathbf{A}_{k}\right)^{-1} \sum_{k=1}^{d}\left(X_{k}-I_{m} \otimes Y_{k}\right) \mathbf{B}_{k} .
$$

Moreover, using the realization $\mathcal{R}_{\mathcal{F M}}$ we can evaluate $\mathfrak{R}$ on every matrix point in the domain of regularity of $\mathfrak{R}$ as well as w.r.t any unital stably finite $\mathbb{K}$-algebra.

The strength of Theorem 3.3 is that we can evaluate any nc rational function on all of its domain and also w.r.t any unital stably finite $\mathbb{K}$-algebra, by using a minimal realization of any nc rational expression which represents the function, that is centred at any point from its domain. As a corollary (Corollary 3.4) we provide a proof of Theorem 1 which - unlike the original proof in [49] — does not make any use of the difference-differential calculus of nc functions, but only the results from Sections 2 and 3.

Generalizations of the main results from Sections 2 and 3 to the matrix valued nc rational functions are briefly summarized in Section 4.

Finally, in Section 5 we provide a full and precise parameterization ((5.3) in Theorem 5.2) of hermitian nc rational functions in terms of their minimal nc FornasiniMarchesini realizations centred at a matrix point. A short discussion and some parameterizations are given for descriptor realizations as well. 
One of the difficulties which arises when moving from a scalar to a matrix centre, is that a minimal nc Fornasini-Marchesini realization $\mathcal{R}_{\mathcal{F} \mathcal{M}}$ of a nc rational expression is no longer a nc rational expression by itself (cf. Remark 2.2). However, in the sequel paper [63], we show that under some constraints (called the linearized lost abbey conditions) on the coefficients of the realization- which follow immediately when $\mathcal{R}_{\mathcal{F} \mathcal{M}}$ is a minimal nc Fornasini-Marchesini realization of a nc rational expression - $\mathcal{R}_{\mathcal{F} \mathcal{M}}$ is actually the restriction of a nc rational function $\mathfrak{R}$ with $D O M_{s}\left(\mathcal{R}_{\mathcal{F M}}\right)=\operatorname{dom}_{s}(\mathfrak{R})$. This will imply the opposite inclusion of the domains in Theorem 2 and thereby complete the proof that the domain of a nc rational function coincides with the domain of any of its minimal realizations, centred at an arbitrary matrix point. As a corollary, also in [63], we will prove that the domain of a nc rational function is equal to its stable extended domain. In a slightly different direction, we will use the the theory of realizations with a matrix centre developed in this paper, together with the results from [63], to present an explicit construction of the free skew field $\mathbb{K} \nless \underline{x} \ngtr$, with a self-contained proof that it is the universal skew field of fractions of the ring of nc polynomials. Moreover, we will construct a functional model and use it to provide a different one step proof for the existence of a realization formula for nc rational functions, without using synthesis. Furthermore, we will establish a generalization of the Kronecker-Fliess theorem, which gives a full characterization of nc rational functions in terms of their formal nc generalized power series expansions around a matrix point. These results will appear in [64].

Finally, we point out that instead of working with Fornasini-Marchesini realizations (for the settings in the commutative original version see $[33,34]$ ) one can consider structured realizations as in [8] and obtain similar results. This is true also for descriptor realizations; for more details see Remark 5.5.

Acknowledgments. The authors would like to thank Joseph Ball, Bill Helton, Dmitry Kalyuzhnyi-Verbovetskyi, Roland Speicher and Juri Volčič for their helpful comments and discussions. The authors would also like to thank the referees for their valuable comments which helped to improve the paper.

\section{Preliminaries}

Notations: $d$ will stand for the number of non-commuting variables, which will be usually denoted by $x_{1}, \ldots, x_{d}$, we often abbreviate non-commuting by nc. For a positive integer $d$, we denote by $\mathcal{G}_{d}$ the free monoid generated by $d$ generators $g_{1}, \ldots, g_{d}$, we say that a word $\omega=g_{i_{1}} \ldots g_{i_{\ell}} \in \mathcal{G}_{d}$ is of length $|\omega|=\ell$ if $\ell \geq 1$ and $\omega=\emptyset$ is of length 0 . For a field $\mathbb{K}$ and $n \in \mathbb{N}$, let $\mathbb{K}^{n \times n}$ be the vector space of $n \times n$ matrices over $\mathbb{K}$, let $\left\{\underline{e}_{1}, \ldots, \underline{e}_{n}\right\}$ be the standard basis of $\mathbb{K}^{n}$ and let $\mathcal{E}_{n}=\left\{E_{i j}=\underline{e}_{i} \underline{e}_{j}^{T}: 1 \leq i, j \leq n\right\}$ be the standard basis of $\mathbb{K}^{n \times n}$. The tensor (Kronecker) product of two matrices $P \in \mathbb{K}^{n_{1} \times n_{2}}$ and $Q \in \mathbb{K}^{n_{3} \times n_{4}}$ is the $n_{1} n_{3} \times n_{2} n_{4}$ block matrix $P \otimes Q=\left[p_{i j} Q\right]_{1 \leq i \leq n_{1}, 1 \leq j \leq n_{2}}$. The range of a matrix $P$, that is the span of all of its columns, denoted by $\operatorname{Im}(P)$.

We denote operators on matrices by bold letters such as $\mathbf{A}, \mathbf{B}$, and the action of $\mathbf{A}$ on $X$ by $\mathbf{A}(X)$. If $\mathbf{A}$ is defined on $s \times s$ matrices we extend $\mathbf{A}$ to act on $s m \times s m$ matrices for any $m \in \mathbb{N}$, by viewing an $s m \times s m$ matrix $X$ as an $m \times m$ matrix with $s \times s$ blocks and by evaluating $\mathbf{A}$ on the $s \times s$ blocks; In that case we 
denote the evaluation by $(X) \mathbf{A}$. If $C$ is a constant matrix and $\mathbf{A}$ is an operator, then $C \cdot \mathbf{A}$ and $\mathbf{A} \cdot C$ are two operators, defined by $(C \cdot \mathbf{A})(X):=C \mathbf{A}(X)$ and $(\mathbf{A} \cdot C)(X):=\mathbf{A}(X) C$. For every $n_{1}, n_{2} \in \mathbb{N}$, we define the permutation matrix

$$
E\left(n_{1}, n_{2}\right)=\left[E_{i j}^{T}\right]_{1 \leq i \leq n_{1}, 1 \leq j \leq n_{2}} \in \mathbb{K}^{n_{1} n_{2} \times n_{1} n_{2}}
$$

and use these matrices to change the order of factors in the Kronecker product of two matrices by the following rule

$$
P \otimes Q=E\left(n_{1}, n_{3}\right)(Q \otimes P) E\left(n_{2}, n_{4}\right)^{T},
$$

for all $n_{1}, n_{2}, n_{3}, n_{4} \in \mathbb{N}, Q \in \mathbb{K}^{n_{1} \times n_{2}}$ and $P \in \mathbb{K}^{n_{3} \times n_{4}}$; for more details see [44, pp. 259-261]. If $P=\left[P_{i j}\right]_{1 \leq i, j \leq m} \in\left(\mathbb{K}^{s \times s}\right)^{m \times m}$ and $Q=\left[Q_{i j}\right]_{1 \leq i, j \leq m} \in\left(\mathbb{K}^{s \times s}\right)^{m \times m}$, then we use the notation

$$
P \odot_{s} Q:=\left[\sum_{k=1}^{m} P_{i k} \otimes Q_{k j}\right]_{1 \leq i, j \leq m}
$$

for the so-called faux product of $P$ and $Q$, viewed as $m \times m$ matrices over the tensor algebra of $\mathbb{K}^{s \times s}$, where $P_{i k} \otimes Q_{k j}$ denotes the element of $\mathbb{K}^{s \times s} \otimes \mathbb{K}^{s \times s}$, rather than the Kronecker product of the matrices; see [60, page 241] for the exact definition and [29] for its origins in operator spaces. If $\underline{X}=\left(X_{1}, \ldots, X_{d}\right) \in\left(\mathbb{K}^{s m \times s m}\right)^{d}$ and $\omega=g_{i_{1}} \ldots g_{i_{\ell}}$, then

$$
\underline{X}^{\odot_{s} \omega}:=X_{i_{1}} \odot_{s} \cdots \odot_{s} X_{i_{\ell}} .
$$

We use $\mathfrak{R}, \mathcal{R}, R$ and $\mathfrak{r}$ for nc rational function, nc Fornasini-Marchesini realization, nc rational expression and matrix valued nc rational function, respectively. Likewise, we use $\mathfrak{a}$ to denote elements in an algebra $\mathcal{A}$ and $\mathfrak{A}$ to denote matrices over $\mathcal{A}$. Throughout the paper, we use underline to denote vectors or $d$-tuples.

1.1. $\mathbf{N C}$ rational functions. If $\mathcal{V}$ is a vector space over a field $\mathbb{K}$, then $\mathcal{V}_{n c}$, the nc space over $\mathcal{V}$, consists of all square matrices over $\mathcal{V}$, i.e.,

$$
\mathcal{V}_{n c}=\coprod_{n=1}^{\infty} \mathcal{V}^{n \times n}
$$

For every $\Omega \subseteq \mathcal{V}_{n c}$ and $n \in \mathbb{N}$ we use the notation $\Omega_{n}:=\Omega \cap \mathcal{V}^{n \times n}$. A subset $\Omega \subseteq \mathcal{V}_{n c}$ is called a nc set if it is closed under direct sums, i.e., if $X \in \Omega_{n}, Y \in \Omega_{m}$ then $X \oplus Y:=\left[\begin{array}{cc}X & 0 \\ 0 & Y\end{array}\right] \in \Omega_{n+m}, \forall m, n \in \mathbb{N}$. In the special case where $\mathcal{V}=\mathbb{K}^{d}$, we have the identification

$$
\left(\mathbb{K}^{d}\right)_{n c}=\coprod_{n=1}^{\infty}\left(\mathbb{K}^{d}\right)^{n \times n} \cong \coprod_{n=1}^{\infty}\left(\mathbb{K}^{n \times n}\right)^{d},
$$

that is the nc space of all $d$-tuples of square matrices over $\mathbb{K}$. Let $\mathcal{V}, \mathcal{W}$ be vector spaces over a field $\mathbb{K}$ and $\Omega \subseteq \mathcal{V}_{n c}$ be an nc set, then $f: \Omega \rightarrow \mathcal{W}_{n c}$ is called a nc function if $f$ is graded, i.e., if $n \in \mathbb{N}$ and $X \in \Omega_{n}$, then $f(X) \in \mathcal{W}^{n \times n}$, and

1. $f$ respects direct sums, i.e., if $X, Y \in \Omega$, then $f(X \oplus Y)=f(X) \oplus f(Y)$;

2. $f$ respects similarities, i.e., if $n \in \mathbb{N}, X \in \Omega_{n}$ and $T \in \mathbb{K}^{n \times n}$ is invertible such that $T \cdot X \cdot T^{-1} \in \Omega$, then $f\left(T \cdot X \cdot T^{-1}\right)=T \cdot f(X) \cdot T^{-1}$.

Notice that if $X \in \Omega_{n}$ and $T \in \mathbb{K}^{n \times n}$, by the products $T \cdot X$ and $X \cdot T$ we mean the standard matrix multiplication and we use the action of $\mathbb{K}$ on $\mathcal{V}$. In particular, if $\mathcal{V}=\mathbb{K}^{d}, \underline{X}=\left(X_{1}, \ldots, X_{d}\right) \in\left(\mathbb{K}^{n \times n}\right)^{d}$ and $T \in \mathbb{K}^{n \times n}$, the products are given by

$$
T \cdot \underline{X}:=\left(T X_{1}, \ldots, T X_{d}\right) \text { and } \underline{X} \cdot T:=\left(X_{1} T, \ldots, X_{d} T\right) \text {. }
$$


An important and central example of nc functions are nc rational expressions. We denote by $\mathbb{K}\left\langle x_{1}, \ldots, x_{d}\right\rangle$ the $\mathbb{K}$-algebra of nc polynomials in the $d$ nc variables $x_{1}, \ldots, x_{d}$ over $\mathbb{K}$. We obtain nc rational expressions by applying successive arithmetic operations (addition, multiplication and taking inverse) on $\mathbb{K}\left\langle x_{1}, \ldots, x_{d}\right\rangle$. For a nc rational expression $R$ and $n \in \mathbb{N}$, let $\operatorname{dom}_{n}(R)$ be the set of all $d$-tuples of $n \times n$ matrices over $\mathbb{K}$ for which all the inverses in $R$ exist; the domain of regularity of $R$ is then defined by

$$
\operatorname{dom}(R):=\coprod_{n=1}^{\infty} \operatorname{dom}_{n}(R)
$$

A nc rational expression $R$ is called non-degenerate if $\operatorname{dom}(R) \neq \emptyset$. For example, $R(\underline{x})=\left(x_{2}+\left(1-x_{1}\right)^{-1}\left(x_{3}^{-1} x_{1}-x_{2}\right)\right) x_{1}$ is a nc rational expression in $x_{1}, x_{2}, x_{3}$, while its domain of regularity is given by

$$
\operatorname{dom}(R)=\coprod_{n=1}^{\infty}\left\{\left(X_{1}, X_{2}, X_{3}\right) \in\left(\mathbb{K}^{n \times n}\right)^{3}: \operatorname{det}\left(I_{n}-X_{1}\right), \operatorname{det}\left(X_{3}\right) \neq 0\right\}
$$

Every nc rational expression $R$ is a nc function from $\operatorname{dom}(R) \subseteq\left(\mathbb{K}^{d}\right)_{n c}$ to $\mathbb{K}_{n c}$. For a detailed discussion of nc rational expressions and their domains of regularity, see [47].

What comes now is the definition of a nc rational function. Let $R_{1}$ and $R_{2}$ be nc rational expressions in $x_{1}, \ldots, x_{d}$ over $\mathbb{K}$. We say that $R_{1}$ and $R_{2}$ are $\left(\mathbb{K}^{d}\right)_{n c}$-evaluation equivalent, if $R_{1}(\underline{X})=R_{2}(\underline{X})$ for every $\underline{X} \in \operatorname{dom}\left(R_{1}\right) \cap \operatorname{dom}\left(R_{2}\right)$. A nc rational function is an equivalence class of non-degenerate nc rational expressions. For every nc rational function $\mathfrak{R}$, define its domain of regularity

$$
\operatorname{dom}(\mathfrak{R}):=\bigcup_{R \in \mathfrak{R}} \operatorname{dom}(R) .
$$

The $\mathbb{K}$-algebra of all nc rational functions of $x_{1}, \ldots, x_{d}$ over $\mathbb{K}$ is denoted by $\mathbb{K} \nless x_{1}, \ldots, x_{d} \ngtr$ and it is a skew field, called the free skew field. Moreover, $\mathbb{K} \nless x_{1}, \ldots, x_{d} \ngtr$ is the universal skew field of fractions of $\mathbb{K}\left\langle x_{1}, \ldots, x_{d}\right\rangle$. See $[6,17,21,22,66]$ for the original proofs and [26] for a more modern reference, while a proof of the equivalence with the evaluations over matrices is presented in $[48,49]$.

1.2. Evaluations over algebras. Let $\mathcal{A}$ be a unital $\mathbb{K}$-algebra. If $\underline{\mathfrak{a}}=\left(\mathfrak{a}_{1}, \ldots, \mathfrak{a}_{d}\right) \in$ $\mathcal{A}^{d}$ and $\omega=g_{i_{1}} \ldots g_{i_{\ell}} \in \mathcal{G}_{d}$, then we use the notations $\underline{\mathfrak{a}}^{\omega}:=\mathfrak{a}_{i_{1}} \cdots \mathfrak{a}_{i_{\ell}}$ and $\underline{\mathfrak{a}}^{\emptyset}=1_{\mathcal{A}}$, where $1_{\mathcal{A}}$ is the unit element in $\mathcal{A}$. We recall the definitions of evaluation and domain of nc rational expressions over $\mathcal{A}$. For more details see [41].

Definition 1.1 ( $\mathcal{A}$-Domains and Evaluations). For any nc rational expression $R$ in $x_{1}, \ldots, x_{d}$ over $\mathbb{K}$, its $\mathcal{A}$-domain $\operatorname{dom}^{\mathcal{A}}(R) \subseteq \mathcal{A}^{d}$ and its evaluation $R^{\mathcal{A}}(\underline{\mathfrak{a}})$ at any $\underline{\mathfrak{a}}=\left(\mathfrak{a}_{1}, \ldots, \mathfrak{a}_{d}\right) \in \operatorname{dom}^{\mathcal{A}}(R)$ are defined by:

1. If $R=\sum_{\omega \in \mathcal{G}_{d}} r_{\omega} \underline{x}^{\omega}$ is a nc polynomial $\left(r_{\omega} \in \mathbb{K}\right)$, then

$$
\operatorname{dom}^{\mathcal{A}}(R)=\mathcal{A}^{d} \text { and } R^{\mathcal{A}}(\underline{\mathfrak{a}})=\sum_{\omega \in \mathcal{G}_{d}} r_{\omega} \underline{\mathfrak{a}}^{\omega} .
$$

2. If $R=R_{1} R_{2}$ where $R_{1}$ and $R_{2}$ are nc rational expressions, then

$$
\operatorname{dom}^{\mathcal{A}}(R)=\operatorname{dom}^{\mathcal{A}}\left(R_{1}\right) \cap \operatorname{dom}^{\mathcal{A}}\left(R_{2}\right) \text { and } R^{\mathcal{A}}(\underline{\mathfrak{a}})=R_{1}^{\mathcal{A}}(\underline{\mathfrak{a}}) R_{2}^{\mathcal{A}}(\underline{\mathfrak{a}}) \text {. }
$$


3. If $R=R_{1}+R_{2}$ where $R_{1}$ and $R_{2}$ are nc rational expressions, then $\operatorname{dom}^{\mathcal{A}}(R)=\operatorname{dom}^{\mathcal{A}}\left(R_{1}\right) \cap \operatorname{dom}^{\mathcal{A}}\left(R_{2}\right)$ and $R^{\mathcal{A}}(\underline{\mathfrak{a}})=R_{1}^{\mathcal{A}}(\underline{\mathfrak{a}})+R_{2}^{\mathcal{A}}(\underline{\mathfrak{a}})$.

4. If $R=R_{1}^{-1}$ where $R_{1}$ is a nc rational expression, then

$$
\operatorname{dom}^{\mathcal{A}}(R)=\left\{\underline{\mathfrak{a}} \in \operatorname{dom}^{\mathcal{A}}\left(R_{1}\right): R_{1}^{\mathcal{A}}(\underline{\mathfrak{a}}) \text { invertible in } \mathcal{A}\right\} \text { and } R^{\mathcal{A}}(\underline{\mathfrak{a}})=\left(R_{1}^{\mathcal{A}}(\underline{\mathfrak{a}})\right)^{-1} \text {. }
$$

Remark 1.2. Let $n \in \mathbb{N}$ and consider the $\mathbb{K}$-algebra $\mathcal{A}_{n}=\mathbb{K}^{n \times n}$. Then, it is easily seen that $\operatorname{dom}^{\mathcal{A}_{n}}(R)=\operatorname{dom}_{n}(R)$ and $R(\underline{\mathfrak{A}})=R^{\mathcal{A}_{n}}(\underline{\mathfrak{A}})$ for every $\underline{\mathfrak{A}} \in \operatorname{dom}_{n}(R)$.

As it will be pointed out later (cf. Theorem 2.15), we are interested in a certain family of algebras, called stably finite algebras. A unital $\mathbb{K}$-algebra $\mathcal{A}$ is called stably finite if for every $m \in \mathbb{N}$ and $\mathfrak{A}, \mathfrak{B} \in \mathcal{A}^{m \times m}$, we have

$$
\mathfrak{A B}=I_{m} \otimes 1_{\mathcal{A}} \Longleftrightarrow \mathfrak{B A}=I_{m} \otimes 1_{\mathcal{A}} .
$$

If $\mathcal{A}$ is a unital $\mathbb{C}^{*}$-algebra with a faithful trace, then $\mathcal{A}$ is stably finite. The following is a characterization of stably finite algebras that we find useful in a later stage of the paper; see [41, Lemma 5.2] for its proof.

Lemma 1.3. Let $\mathcal{A}$ be a unital $\mathbb{K}$-algebra. The following are equivalent:

1. $\mathcal{A}$ is stably finite.

2. For every $n \in \mathbb{N}, m_{1}, \ldots, m_{n} \in \mathbb{N}$ and $\mathfrak{A}_{i, j} \in \mathcal{A}^{m_{i} \times m_{j}}, i, j=1, \ldots, n$, if the upper (or lower) triangular block matrix

$$
\left[\begin{array}{cccc}
\mathfrak{A}_{11} & * & * & * \\
0 & \mathfrak{A}_{22} & * & * \\
\vdots & \ddots & \ddots & * \\
0 & \cdots & 0 & \mathfrak{A}_{n n}
\end{array}\right]
$$

is invertible, then the matrices $\mathfrak{A}_{11}, \ldots, \mathfrak{A}_{n n}$ are invertible.

\section{Realizations of NC Rational Expressions}

Non-commutative Fornasini-Marchesini realizations, see [8, 49] and [33, 34] for the original commutative version, apply to nc rational expressions which are regular at $\underline{0}$. By translation, the point $\underline{0}$ can be replaced by any scalar point. In this section we develop analogous realization formulas for nc rational expressions, centred at an arbitrary matrix point in the domain of regularity of the expression.

Definition 2.1. Let $s, L \in \mathbb{N}, \underline{Y}=\left(Y_{1}, \ldots, Y_{d}\right) \in\left(\mathbb{K}^{s \times s}\right)^{d}$,

$$
\mathbf{A}_{1}, \ldots, \mathbf{A}_{d}: \mathbb{K}^{s \times s} \rightarrow \mathbb{K}^{L \times L} \text { and } \mathbf{B}_{1}, \ldots, \mathbf{B}_{d}: \mathbb{K}^{s \times s} \rightarrow \mathbb{K}^{L \times s}
$$

be linear mappings, $C \in \mathbb{K}^{s \times L}$ and $D \in \mathbb{K}^{s \times s}$. Then

$$
\mathcal{R}\left(X_{1}, \ldots, X_{d}\right)=D+C\left(I_{L}-\sum_{k=1}^{d} \mathbf{A}_{k}\left(X_{k}-Y_{k}\right)\right)^{-1} \sum_{k=1}^{d} \mathbf{B}_{k}\left(X_{k}-Y_{k}\right)
$$

is called a nc Fornasini-Marchesini realization centred at $\underline{Y}$ and it is defined for every $\underline{X}=\left(X_{1}, \ldots, X_{d}\right) \in D O M_{s}(\mathcal{R})$, where

$$
D O M_{s}(\mathcal{R}):=\left\{\underline{X} \in\left(\mathbb{K}^{s \times s}\right)^{d}: \operatorname{det}\left(I_{L}-\sum_{k=1}^{d} \mathbf{A}_{k}\left(X_{k}-Y_{k}\right)\right) \neq 0\right\} .
$$

In that case we say that the realization $\mathcal{R}$ is described by the tuple $(L, D, C, \underline{\mathbf{A}}, \underline{\mathbf{B}})$. 
Remark 2.2. If $s=1$, then $\mathcal{R}$ is a $1 \times 1$ matrix valued nc rational expression (see Remark 4.4 for details) and $D O M_{s}(\mathcal{R})=\operatorname{dom}_{s}(\mathcal{R})$. However, this is not the case for $s>1$ and that is why we use the notation $D_{O} M_{s}(\mathcal{R})$ instead of $\operatorname{dom}_{s}(\mathcal{R})$.

Let $s_{1}, s_{2}, s_{3}, s_{4} \in \mathbb{N}$. If $\mathbf{T}: \mathbb{K}^{s_{1} \times s_{2}} \rightarrow \mathbb{K}^{s_{3} \times s_{4}}$ is a linear mapping and $m \in \mathbb{N}$, then $\mathbf{T}$ can be naturally extended to a linear mapping $\mathbf{T}: \mathbb{K}^{s_{1} m \times s_{2} m} \rightarrow \mathbb{K}^{s_{3} m \times s_{4} m}$, by the following rule:

$$
X=\left[X_{i j}\right]_{1 \leq i, j \leq m} \in \mathbb{K}^{s_{1} m \times s_{2} m} \Longrightarrow(X) \mathbf{T}=\left[\mathbf{T}\left(X_{i j}\right)\right]_{1 \leq i, j \leq m},
$$

i.e., $(X) \mathbf{T}$ is an $m \times m$ block matrix with entries in $\mathbb{K}^{s_{3} \times s_{4}}$. Therefore, we can extend the realization (2.1) to act on $d$-tuples of $s m \times s m$ matrices: for every $\underline{X}=\left(X_{1}, \ldots, X_{d}\right)$ in

$$
D O M_{s m}(\mathcal{R}):=\left\{\underline{X} \in\left(\mathbb{K}^{s m \times s m}\right)^{d}: \operatorname{det}\left(I_{L m}-\sum_{k=1}^{d}\left(X_{k}-I_{m} \otimes Y_{k}\right) \mathbf{A}_{k}\right) \neq 0\right\},
$$

define

$\mathcal{R}(\underline{X}):=I_{m} \otimes D+\left(I_{m} \otimes C\right)\left(I_{L m}-\sum_{k=1}^{d}\left(X_{k}-I_{m} \otimes Y_{k}\right) \mathbf{A}_{k}\right)^{-1} \sum_{k=1}^{d}\left(X_{k}-I_{m} \otimes Y_{k}\right) \mathbf{B}_{k}$.

In addition, if $\mathcal{A}$ is a unital $\mathbb{K}$-algebra, a linear mapping $\mathbf{T}: \mathbb{K}^{s_{1} \times s_{2}} \rightarrow \mathbb{K}^{s_{3} \times s_{4}}$ can be also naturally extended to a linear mapping $\mathbf{T}^{\mathcal{A}}: \mathcal{A}^{s_{1} \times s_{2}} \rightarrow \mathcal{A}^{s_{3} \times s_{4}}$ by the following rule:

$$
\mathfrak{A}=\sum_{i=1}^{s_{1}} \sum_{j=1}^{s_{2}} E_{i j} \otimes \mathfrak{a}_{i j} \in \mathcal{A}^{s_{1} \times s_{2}} \Longrightarrow(\mathfrak{A}) \mathbf{T}^{\mathcal{A}}=\sum_{i=1}^{s_{1}} \sum_{j=1}^{s_{2}} \mathbf{T}\left(E_{i j}\right) \otimes \mathfrak{a}_{i j} \in \mathcal{A}^{s_{3} \times s_{4}},
$$

where $E_{i j}=\underline{e}_{i} \underline{e}_{j}^{T} \in \mathbb{K}^{s_{1} \times s_{2}}$ and $\mathfrak{a}_{i j} \in \mathcal{A}$. If $\mathcal{R}$ is a nc Fornasini-Marchesini realization centred at $\underline{Y}$, as in (2.1), define its $\mathcal{A}$-domain to be the subset of $\left(\mathcal{A}^{s \times s}\right)^{d}$ given by

$D O M^{\mathcal{A}}(\mathcal{R}):=\left\{\underline{\mathfrak{A}} \in\left(\mathcal{A}^{s \times s}\right)^{d}:\left(I_{L} \otimes 1_{\mathcal{A}}-\sum_{k=1}^{d}\left(\mathfrak{A}_{k}-Y_{k} \otimes 1_{\mathcal{A}}\right) \mathbf{A}_{k}^{\mathcal{A}}\right)\right.$ is invertible in $\left.\mathcal{A}^{L \times L}\right\}$

and for every $\underline{\mathfrak{A}}=\left(\mathfrak{A}_{1}, \ldots, \mathfrak{A}_{d}\right) \in D O M^{\mathcal{A}}(\mathcal{R})$ define the evaluation of $\mathcal{R}$ at $\underline{\mathfrak{A}}$ by

$$
\begin{aligned}
\mathcal{R}^{\mathcal{A}}(\underline{\mathfrak{A}}):=D \otimes 1_{\mathcal{A}}+\left(C \otimes 1_{\mathcal{A}}\right)\left(I_{L} \otimes 1_{\mathcal{A}}-\sum_{k=1}^{d}\left[\left(\mathfrak{A}_{k}\right) \mathbf{A}_{k}^{\mathcal{A}}-\mathbf{A}_{k}\left(Y_{k}\right) \otimes 1_{\mathcal{A}}\right]\right)^{-1} \\
\sum_{k=1}^{d}\left[\left(\mathfrak{A}_{k}\right) \mathbf{B}_{k}^{\mathcal{A}}-\mathbf{B}_{k}\left(Y_{k}\right) \otimes 1_{\mathcal{A}}\right] .
\end{aligned}
$$

2.1. Existence. The way we define what is a realization of a nc rational expression is different than the usual definition. In the usual case, the expression and the realization coincide whenever they are both defined, while in our definition we include the fact that the domain of the expression is contained in the domain of the realization. We begin with the definition of a nc rational expression admitting a realization, both in the usual way (over matrices) and in the case of evaluations w.r.t an algebra. 
Definition 2.3. Let $R$ be a nc rational expression in $x_{1}, \ldots, x_{d}$ over $\mathbb{K}, \underline{Y}=$ $\left(Y_{1}, \ldots, Y_{d}\right) \in \operatorname{dom}_{s}(R), \mathcal{R}$ be a nc Fornasini-Marchesini realization centred at $\underline{Y}$ and $\mathcal{A}$ be a unital $\mathbb{K}$-algebra. We say that:

1. $R$ admits the realization $\mathcal{R}$, or that $\mathcal{R}$ is a realization of $R$, if

$$
\operatorname{dom}_{s m}(R) \subseteq D O M_{s m}(\mathcal{R}) \text { and } R(\underline{X})=\mathcal{R}(\underline{X}), \forall \underline{X} \in \operatorname{dom}_{s m}(\mathcal{R})
$$

for every $m \in \mathbb{N}$.

2. $R$ admits the realization $\mathcal{R}$ with respect to (w.r.t) $\mathcal{A}$, or that $\mathcal{R}$ is a realization of $R$ w.r.t $\mathcal{A}$, if for every $\underline{\mathfrak{a}}=\left(\mathfrak{a}_{1}, \ldots, \mathfrak{a}_{d}\right) \in \operatorname{dom}^{\mathcal{A}}(R)$ :

$$
I_{s} \otimes \underline{\mathfrak{a}}:=\left(I_{s} \otimes \mathfrak{a}_{1}, \ldots, I_{s} \otimes \mathfrak{a}_{d}\right) \in \operatorname{DOM}^{\mathcal{A}}(\mathcal{R})
$$

and $I_{s} \otimes R^{\mathcal{A}}(\underline{\mathfrak{a}})=\mathcal{R}^{\mathcal{A}}\left(I_{s} \otimes \underline{\mathfrak{a}}\right)$.

We begin by showing the existence of a nc Fornasini-Marchesini realization for every nc rational expression $R$, centred at any $\underline{Y} \in \operatorname{dom}_{s}(R)$, that is also a realization of $R$ w.r.t any unital $\mathbb{K}$-algebra.

Theorem 2.4. Let $R$ be a nc rational expression in $x_{1}, \ldots, x_{d}$ over $\mathbb{K}$ and let $\underline{Y}=\left(Y_{1}, \ldots, Y_{d}\right) \in \operatorname{dom}_{s}(R)$. There exists a nc Fornasini-Marchesini realization $\overline{\mathcal{R}}$ of $R$ centred at $\underline{Y}$, such that $\mathcal{R}$ is a realization of $R$ w.r.t any unital $\mathbb{K}$-algebra.

The proof is done by synthesis, which is going back to ideas from automata theory $[18,72,73]$ and system theory $[27,28]$. We also use the following technical fact: let $\underline{X}=\left(X_{1}, \ldots, X_{d}\right) \in\left(\mathbb{K}^{s m \times s m}\right)^{d}$ and write

$$
X_{k}=\sum_{i, j=1}^{m} E_{i j} \otimes X_{i j}^{(k)}, \text { with } E_{i j} \in \mathbb{K}^{m \times m}, X_{i j}^{(k)} \in \mathbb{K}^{s \times s}, 1 \leq k \leq d
$$

then $\left(X_{k}-I_{m} \otimes Y_{k}\right) \mathbf{A}_{k}=\sum_{i, j=1}^{m} E_{i j} \otimes \mathbf{A}_{k}\left(X_{i j}^{(k)}\right)-I_{m} \otimes \mathbf{A}_{k}\left(Y_{k}\right)$ and hence

$$
\left(X_{k}-I_{m} \otimes Y_{k}\right) \mathbf{A}_{k}=P_{2}\left(\sum_{i, j=1}^{m} \mathbf{A}_{k}\left(X_{i j}^{(k)}\right) \otimes E_{i j}-\mathbf{A}_{k}\left(Y_{k}\right) \otimes I_{m}\right) P_{2}^{-1}
$$

and similarly

$$
\left(X_{k}-I_{m} \otimes Y_{k}\right) \mathbf{B}_{k}=P_{2}\left(\sum_{i, j=1}^{m} \mathbf{B}_{k}\left(X_{i j}^{(k)}\right) \otimes E_{i j}-\mathbf{B}_{k}\left(Y_{k}\right) \otimes I_{m}\right) P_{1}^{-1},
$$

where $P_{1}=E(m, s)$ and $P_{2}=E(m, L)$ are the shuffle matrices defined in (1.1). Therefore for every $\underline{X} \in D O M_{s m}(\mathcal{R})$ we have

$$
\begin{aligned}
& P_{1}^{-1} \mathcal{R}(\underline{X}) P_{1}=D \otimes I_{m}+P_{1}^{-1}\left(I_{m} \otimes C\right) P_{2}\left(I_{L m}-\sum_{k=1}^{d}\left[\sum_{i, j=1}^{m} \mathbf{A}_{k}\left(X_{i j}^{(k)}\right) \otimes E_{i j}-\mathbf{A}_{k}\left(Y_{k}\right) \otimes I_{m}\right]\right)^{-1} \\
& \sum_{k=1}^{d}\left[\sum_{i, j=1}^{m} \mathbf{B}_{k}\left(X_{i j}^{(k)}\right) \otimes E_{i j}-\mathbf{B}_{k}\left(Y_{k}\right) \otimes I_{m}\right]=D \otimes I_{m}+\left(C \otimes I_{m}\right)\left(I_{L m}-\sum_{k=1}^{d}\left[\sum_{i, j=1}^{m}\right.\right. \\
& \left.\left.\mathbf{A}_{k}\left(X_{i j}^{(k)}\right) \otimes E_{i j}-\mathbf{A}_{k}\left(Y_{k}\right) \otimes I_{m}\right]\right)^{-1} \sum_{k=1}^{d}\left[\sum_{i, j=1}^{m} \mathbf{B}_{k}\left(X_{i j}^{(k)}\right) \otimes E_{i j}-\mathbf{B}_{k}\left(Y_{k}\right) \otimes I_{m}\right] .
\end{aligned}
$$


Proof. We first show that the theorem is true for all monomials $x_{1}, \ldots, x_{d}$ and constants, then we show that if it is true for two rational expressions, so it is also true for their summation, multiplication and their inversion, when they exist.

1. Constants: Let $R_{0}(\underline{x})=K \in \mathbb{K}$. If $\underline{\mathfrak{a}} \in \operatorname{dom}^{\mathcal{A}}\left(R_{0}\right)=\mathcal{A}^{d}$, then $I_{s} \otimes \underline{\mathfrak{a}} \in$

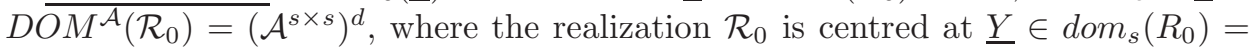
$\left(\mathbb{K}^{s \times s}\right)^{d}$ and described by

$$
L=1, D=I_{s} \otimes K, C=0, \mathbf{A}_{1}=\ldots=\mathbf{A}_{d}=0, \mathbf{B}_{1}=\ldots=\mathbf{B}_{d}=0
$$

and $I_{s} \otimes R_{0}^{\mathcal{A}}(\underline{\mathfrak{a}})=I_{s} \otimes\left(K \otimes 1_{\mathcal{A}}\right)=D \otimes 1_{\mathcal{A}}=\mathcal{R}_{0}^{\mathcal{A}}\left(I_{s} \otimes \underline{\mathfrak{a}}\right)$. Moreover,

$$
\operatorname{dom}_{s m}\left(R_{0}\right)=\left(\mathbb{K}^{s m \times s m}\right)^{d}=D O M_{s m}\left(\mathcal{R}_{0}\right)
$$

and for every $\underline{X} \in \operatorname{dom}_{s m}\left(R_{0}\right)$ we have $R_{0}(\underline{X})=I_{s m} \otimes K=I_{m} \otimes D=\mathcal{R}_{0}(\underline{X})$.

2. Monomials: Let $R_{j}(\underline{x})=x_{j}$ for $1 \leq j \leq d$. If $\underline{\mathfrak{a}} \in \operatorname{dom}^{\mathcal{A}}\left(R_{j}\right)=\mathcal{A}^{d}$, then $I_{s} \otimes \underline{\mathfrak{a}} \in D O M^{\mathcal{A}}\left(\mathcal{R}_{j}\right)=\left(\mathcal{A}^{s \times s}\right)^{d}$, where the realization $\mathcal{R}_{j}$ is centred at $\underline{Y} \in \operatorname{dom}_{s}\left(R_{j}\right)=\left(\mathbb{K}^{s \times s}\right)^{d}$ and described by

$$
L=s, D=Y_{j}, C=I_{s}, \mathbf{A}_{1}=\ldots=\mathbf{A}_{d}=0, \mathbf{B}_{j}=I d, \mathbf{B}_{k}=0(\forall k \neq j)
$$

and

$$
I_{s} \otimes R_{j}^{\mathcal{A}}(\underline{\mathfrak{a}})=I_{s} \otimes \mathfrak{a}_{j}=Y_{j} \otimes 1_{\mathcal{A}}+\left(I_{s} \otimes \mathfrak{a}_{j}-Y_{j} \otimes 1_{\mathcal{A}}\right) \mathbf{B}_{j}^{\mathcal{A}}=\mathcal{R}_{j}^{\mathcal{A}}\left(I_{s} \otimes \underline{\mathfrak{a}}\right) .
$$

Moreover, $\operatorname{dom}_{s m}\left(R_{j}\right)=\left(\mathbb{K}^{s m \times s m}\right)^{d}=D O M_{s m}\left(\mathcal{R}_{j}\right)$ and for every $\underline{X} \in \operatorname{dom}_{s m}\left(R_{j}\right)$ we have $R(\underline{X})=X_{j}=I_{m} \otimes Y_{j}+\left(X_{j}-I_{m} \otimes Y_{j}\right) \mathbf{B}_{j}=\mathcal{R}_{j}(\underline{X})$.

3. Addition: Suppose $R_{1}$ and $R_{2}$ are two nc rational expressions admitting realizations $\mathcal{R}_{1}$ and $\mathcal{R}_{2}$ both centred at $\underline{Y}$, described by the tuples $\left(L_{1}, D^{1}, C^{1}, \underline{\mathbf{A}}^{1}, \underline{\mathbf{B}}^{1}\right)$ and $\left(L_{2}, D^{2}, C^{2}, \underline{\mathbf{A}}^{2}, \underline{\mathbf{B}}^{2}\right)$, respectively, and also w.r.t any unital $\mathbb{K}$-algebra $\mathcal{A}$.

Thus, $\underline{\mathfrak{a}} \in \operatorname{dom}^{\mathcal{A}}\left(R_{1}+R_{2}\right)=\operatorname{dom}^{\mathcal{A}}\left(R_{1}\right) \cap \operatorname{dom}^{\mathcal{A}}\left(R_{2}\right)$ implies that $I_{s} \otimes \underline{\mathfrak{a}} \in$ $D O M^{\mathcal{A}}\left(\overline{\mathcal{R}}_{1}\right) \cap D O M^{\mathcal{A}}\left(\mathcal{R}_{2}\right)$,

$$
\begin{array}{r}
I_{s} \otimes\left(R_{1}+R_{2}\right)^{\mathcal{A}}(\underline{\mathfrak{a}})=\mathcal{R}_{1}^{\mathcal{A}}\left(I_{s} \otimes \underline{\mathfrak{a}}\right)+\mathcal{R}_{2}^{\mathcal{A}}\left(I_{s} \otimes \underline{\mathfrak{a}}\right)=\left(\mathcal{R}_{1}^{\mathcal{A}}+\mathcal{R}_{2}^{\mathcal{A}}\right)\left(I_{s} \otimes \underline{\mathfrak{a}}\right)=D^{\mathrm{par}} \otimes 1_{\mathcal{A}} \\
+\left(C^{\mathrm{par}} \otimes 1_{\mathcal{A}}\right)\left(I_{L} \otimes 1_{\mathcal{A}}-\sum_{k=1}^{d}\left[\mathbf{A}_{k}^{\mathrm{par}}\left(I_{s}\right) \otimes \mathfrak{a}_{k}-\mathbf{A}_{k}^{\mathrm{par}}\left(Y_{k}\right) \otimes 1_{\mathcal{A}}\right]\right)^{-1} \sum_{k=1}^{d}\left[\mathbf{B}_{k}^{\mathrm{par}}\left(I_{s}\right) \otimes \mathfrak{a}_{k}-\right. \\
\left.\mathbf{B}_{k}^{\mathrm{par}}\left(Y_{k}\right) \otimes 1_{\mathcal{A}}\right]:=\left(\mathcal{R}^{\mathrm{par}}\right)^{\mathcal{A}}\left(I_{s} \otimes \underline{\mathfrak{a}}\right)
\end{array}
$$

and $I_{s} \otimes \underline{\mathfrak{a}} \in D O M^{\mathcal{A}}\left(\mathcal{R}^{\text {par }}\right)$, when $\mathcal{R}^{\text {par }}$ is the nc Fornasini-Marchesini realization centred at $\underline{Y}$ described by

$$
\begin{array}{r}
L=L_{1}+L_{2}, D^{\text {par }}=D^{1}+D^{2}, C^{\text {par }}=\left[\begin{array}{ll}
C^{1} & C^{2}
\end{array}\right], \mathbf{A}_{k}^{\mathrm{par}}=\left[\begin{array}{cc}
\mathbf{A}_{k}^{1} & 0 \\
0 & \mathbf{A}_{k}^{2}
\end{array}\right] \\
\text { and } \mathbf{B}_{k}^{\text {par }}=\left[\begin{array}{l}
\mathbf{B}_{k}^{1} \\
\mathbf{B}_{k}^{2}
\end{array}\right], k=1, \ldots, d
\end{array}
$$

Also, for every $m \in \mathbb{N}, \underline{X} \in \operatorname{dom}_{s m}\left(R_{1}+R_{2}\right)=\operatorname{dom}_{s m}\left(R_{1}\right) \cap \operatorname{dom}_{s m}\left(R_{2}\right)$ implies $\underline{X} \in D O M_{s m}\left(\mathcal{R}_{i}\right)$ and $R_{i}(\underline{X})=\mathcal{R}_{i}(\underline{X})$ for $i=1,2$ and hence $\left(R_{1}+R_{2}\right)(\underline{X})=$ $\overline{\mathcal{R}}_{1}(\underline{X})+\mathcal{R}_{2}(\underline{X})$. Write $\underline{X}=\left(X_{1}, \ldots, X_{d}\right) \in\left(\mathbb{K}^{s m \times s m}\right)^{d}$ and use (2.3) to obtain 
that

$$
\begin{gathered}
P_{1}^{-1}\left(\mathcal{R}_{1}(\underline{X})+\mathcal{R}_{2}(\underline{X})\right) P_{1}=D^{\mathrm{par}} \otimes I_{m}+\left(C^{\mathrm{par}} \otimes I_{m}\right)\left(I_{L m}-\sum_{k=1}^{d}\left[\sum_{i, j=1}^{m} \mathbf{A}_{k}^{\mathrm{par}}\left(X_{i j}^{(k)}\right)\right.\right. \\
\left.\left.\otimes E_{i j}-\mathbf{A}_{k}^{\mathrm{par}}\left(Y_{k}\right) \otimes I_{m}\right]\right)^{-1} \sum_{k=1}^{d}\left[\sum_{i, j=1}^{m} \mathbf{B}_{k}^{\mathrm{par}}\left(X_{i j}^{(k)}\right) \otimes E_{i j}-\mathbf{B}_{k}^{\mathrm{par}}\left(Y_{k}\right) \otimes I_{m}\right]=D^{\mathrm{par}} \otimes I_{m} \\
+\left(C^{\mathrm{par}} \otimes I_{m}\right) P_{2}^{-1}\left(I_{L m}-\sum_{k=1}^{d}\left[\sum_{i, j=1}^{m} E_{i j} \otimes \mathbf{A}_{k}^{\mathrm{par}}\left(X_{i j}^{(k)}\right)-I_{m} \otimes \mathbf{A}_{k}^{\mathrm{par}}\left(Y_{k}\right)\right]\right)^{-1} P_{2} P_{2}^{-1}\left(\sum_{k=1}^{d}\right. \\
\left.\left[\sum_{i, j=1}^{m} E_{i j} \otimes \mathbf{B}_{k}^{\mathrm{par}}\left(X_{i j}^{(k)}\right)-I_{m} \otimes \mathbf{B}_{k}^{\mathrm{par}}\left(Y_{k}\right)\right]\right) P_{1}=P_{1}^{-1} \mathcal{R}^{\mathrm{par}}(\underline{X}) P_{1},
\end{gathered}
$$

i.e., $\mathcal{R}_{1}(\underline{X})+\mathcal{R}_{2}(\underline{X})=\mathcal{R}^{\mathrm{par}}(\underline{X})$ while it is easily seen that $\underline{X} \in D O M_{s m}\left(\mathcal{R}^{\mathrm{par}}\right)$.

4. Multiplication: Suppose $R_{1}$ and $R_{2}$ are two nc rational expressions admitting realizations $\mathcal{R}_{1}$ and $\mathcal{R}_{2}$ both centred at $\underline{Y}$, described by the tuples $\left(L_{1}, D^{1}, C^{1}, \underline{\mathbf{A}}^{1}, \underline{\mathbf{B}}^{1}\right)$ and $\left(L_{2}, D^{2}, C^{2}, \underline{\mathbf{A}}^{2}, \underline{\mathbf{B}}^{2}\right)$, respectively, and also w.r.t any unital $\mathbb{K}$-algebra $\mathcal{A}$.

Thus, $\underline{\mathfrak{a}} \in \operatorname{dom}^{\mathcal{A}}\left(R_{1} R_{2}\right)=\operatorname{dom}^{\mathcal{A}}\left(R_{1}\right) \cap \operatorname{dom}^{\mathcal{A}}\left(R_{2}\right)$ implies that $I_{s} \otimes \underline{\mathfrak{a}} \in \operatorname{DOM}^{\mathcal{A}}\left(\mathcal{R}_{1}\right) \cap$ $\operatorname{DOM}^{\mathcal{A}}\left(\mathcal{R}_{2}\right)$,

$$
\begin{array}{r}
I_{s} \otimes\left(R_{1} R_{2}\right)^{\mathcal{A}}(\underline{\mathfrak{a}})=\mathcal{R}_{1}^{\mathcal{A}}\left(I_{s} \otimes \underline{\mathfrak{a}}\right) \mathcal{R}_{2}^{\mathcal{A}}\left(I_{s} \otimes \underline{\mathfrak{a}}\right)=\left(\mathcal{R}_{1} \mathcal{R}_{2}\right)^{\mathcal{A}}\left(I_{s} \otimes \underline{\mathfrak{a}}\right)=D^{\mathrm{ser}} \otimes 1_{\mathcal{A}}+\left(C^{\mathrm{ser}} \otimes 1_{\mathcal{A}}\right) \\
\left(I_{L} \otimes 1_{\mathcal{A}}-\sum_{k=1}^{d}\left[\mathbf{A}_{k}^{\mathrm{ser}}\left(I_{s}\right) \otimes \mathfrak{a}_{k}-\mathbf{A}_{k}^{\mathrm{ser}}\left(Y_{k}\right) \otimes 1_{\mathcal{A}}\right]\right)^{-1} \sum_{k=1}^{d}\left[\mathbf{B}_{k}^{\mathrm{ser}}\left(I_{s}\right) \otimes \mathfrak{a}_{k}-\mathbf{B}_{k}^{\mathrm{ser}}\left(Y_{k}\right) \otimes 1_{\mathcal{A}}\right] \\
:=\left(\mathcal{R}^{\mathrm{ser}}\right)^{\mathcal{A}}\left(I_{s} \otimes \underline{\mathfrak{a}}\right)
\end{array}
$$

and $I_{s} \otimes \underline{\mathfrak{a}} \in D O M^{\mathcal{A}}\left(\mathcal{R}^{\text {ser }}\right)$, when $\mathcal{R}^{\text {ser }}$ is the nc Fornasini-Marchesini realization centred at $\underline{Y}$ described by

$$
\begin{array}{r}
L=L_{1}+L_{2}, D^{\text {ser }}=D^{1} D^{2}, C^{\text {ser }}=\left[\begin{array}{ll}
C^{1} & D^{1} C^{2}
\end{array}\right], \mathbf{A}_{k}^{\text {ser }}=\left[\begin{array}{cc}
\mathbf{A}_{k}^{1} & \mathbf{B}_{k}^{1} \cdot C^{2} \\
0 & \mathbf{A}_{k}^{2}
\end{array}\right] \\
\text { and } \mathbf{B}_{k}^{\mathrm{ser}}=\left[\begin{array}{c}
\mathbf{B}_{k}^{1} \cdot D^{2} \\
\mathbf{B}_{k}^{2}
\end{array}\right], k=1, \ldots, d .
\end{array}
$$

Also, for every $m \in \mathbb{N}, \underline{X} \in \operatorname{dom}_{s m}\left(R_{1} R_{2}\right)=\operatorname{dom}_{s m}\left(R_{1}\right) \cap \operatorname{dom}_{s m}\left(R_{2}\right)$ implies that $\underline{X} \in D O M_{s m}\left(\mathcal{R}_{i}\right)$ and $R_{i}(\underline{X})=\mathcal{R}_{i}(\underline{X})$ for $i=1,2$ and $\left(R_{1} R_{2}\right)(\underline{X})=$ $\mathcal{R}_{1}(\underline{X}) \mathcal{R}_{2}(\underline{X})$. Now, let $\underline{X}=\left(X_{1}, \ldots, X_{d}\right) \in\left(\mathbb{K}^{s m \times s m}\right)^{d}$ as in (2.2), so similar computation shows that $\underline{X} \in D O M_{s m}(\mathcal{R})$ and $\left(R_{1} R_{2}\right)(\underline{X})=\mathcal{R}_{1}(\underline{X}) \mathcal{R}_{2}(\underline{X})=\mathcal{R}^{\mathrm{ser}}(\underline{X})$.

5. Inverses: Suppose $R$ is a nc rational expression admitting a realization $\mathcal{R}$ centred at $\underline{Y}$, described by the tuple $(L, D, C, \underline{\mathbf{A}}, \underline{\mathbf{B}})$, also w.r.t any unital $\mathbb{K}$-algebra $\mathcal{A}$ and $R(\underline{Y})=D$ is invertible.

Thus, $\underline{\mathfrak{a}} \in \operatorname{dom}^{\mathcal{A}}\left(R^{-1}\right)$ implies that $\underline{\mathfrak{a}} \in \operatorname{dom}^{\mathcal{A}}(R), I_{s} \otimes \underline{\mathfrak{a}} \in \operatorname{DOM}^{\mathcal{A}}(\mathcal{R}), R^{\mathcal{A}}(\underline{\mathfrak{a}})$ is invertible and $\mathcal{R}^{\mathcal{A}}\left(I_{s} \otimes \underline{\mathfrak{a}}\right)=I_{s} \otimes R^{\mathcal{A}}(\underline{\mathfrak{a}})$, so

$$
I_{s} \otimes\left(R^{-1}\right)^{\mathcal{A}}(\underline{\mathfrak{a}})=\left(I_{s} \otimes R^{\mathcal{A}}(\underline{\mathfrak{a}})\right)^{-1}=\left(\mathcal{R}^{\mathcal{A}}\left(I_{s} \otimes \underline{\mathfrak{a}}\right)\right)^{-1}=\left(\mathcal{R}^{\mathrm{inv}}\right)^{\mathcal{A}}\left(I_{s} \otimes \underline{\mathfrak{a}}\right)
$$


and $I_{s} \otimes \underline{\mathfrak{a}} \in D O M^{\mathcal{A}}\left(\mathcal{R}^{\mathrm{inv}}\right)$, when $\mathcal{R}^{\mathrm{inv}}$ is the nc Fornasini-Marchesini realization centred at $\underline{Y}$ described by

$$
\begin{aligned}
D^{\text {inv }}=D^{-1}, C^{\text {inv }}=D^{-1} C, \mathbf{A}_{k}^{\text {inv }}=\mathbf{A}_{k}-\mathbf{B}_{k} \cdot\left(D^{-1} C\right) \text { and } \\
\quad \mathbf{B}_{k}^{\text {inv }}=-\mathbf{B}_{k} \cdot D^{-1}, k=1, \ldots, d .
\end{aligned}
$$

Moreover, if $\underline{X} \in \operatorname{dom}_{s m}\left(R^{-1}\right)$, then $\underline{X} \in \operatorname{dom}_{s m}(R)$ and $R(\underline{X})$ is invertible, so $\underline{X} \in D O M_{s m}(\mathcal{R})$ and $R(\underline{X})=\mathcal{R}(\underline{X})$ is invertible, therefore the matrices $M$ and $I_{m} \otimes D+\left(I_{m} \otimes C\right) M^{-1} N$ are invertible, where

$$
M:=I_{L m}-\sum_{k=1}^{d}\left(X_{k}-I_{m} \otimes Y_{k}\right) \mathbf{A}_{k} \text { and } N:=\sum_{k=1}^{d}\left(X_{k}-I_{m} \otimes Y_{k}\right) \mathbf{B}_{k} .
$$

Consider the matrix

$$
E:=\left[\begin{array}{cc}
-M & N \\
I_{m} \otimes C & I_{m} \otimes D
\end{array}\right] \in \mathbb{K}^{(L+s) m \times(L+s) m}
$$

together with its two Schur complements decompositions

$$
\begin{gathered}
E=\left[\begin{array}{cc}
I_{L m} & 0 \\
-\left(I_{m} \otimes C\right) M^{-1} & I_{s m}
\end{array}\right]\left[\begin{array}{cc}
-M & 0 \\
0 & I_{m} \otimes D+\left(I_{m} \otimes C\right) M^{-1} N
\end{array}\right]\left[\begin{array}{cc}
I_{L m} & -M^{-1} N \\
0 & I_{s m}
\end{array}\right] \\
=\left[\begin{array}{cc}
I_{L m} & N\left(I_{m} \otimes D\right)^{-1} \\
0 & I_{s m}
\end{array}\right]\left[\begin{array}{cc}
-M-N\left(I_{m} \otimes D^{-1} C\right) & 0 \\
0 & I_{m} \otimes D
\end{array}\right]\left[\begin{array}{cc}
I_{L m} & 0 \\
I_{m} \otimes\left(D^{-1} C\right) & I_{s m}
\end{array}\right] .
\end{gathered}
$$

As $M$ and $I_{m} \otimes D+\left(I_{m} \otimes C\right) M^{-1} N$ are invertible, it follows that $E$ is invertible and hence

$$
M+N\left(I_{m} \otimes\left(D^{-1} C\right)\right)=I_{L m}-\sum_{k=1}^{d}\left(X_{k}-I_{m} \otimes Y_{k}\right) \mathbf{A}_{k}^{\mathrm{inv}}
$$

is invertible, i.e., $\underline{X} \in D O M_{s m}\left(\mathcal{R}^{\text {inv }}\right)$. Thus $\operatorname{dom}_{s m}\left(R^{-1}\right) \subseteq D O M_{s m}\left(\mathcal{R}^{\text {inv }}\right)$ and for every $\underline{X} \in \operatorname{dom}_{s m}\left(R^{-1}\right)$, we have $R^{-1}(\underline{X})=\mathcal{R}(\underline{X})^{-1}=\mathcal{R}^{\text {inv }}(\underline{X})$.

We finish this subsection by comparing the two parts of Definition 2.3 for the $\mathbb{K}$-algebra $\mathcal{A}_{n}=\mathbb{K}^{n \times n}$ (cf. Remark 1.2). This will imply (see Corollary 2.6) that for every nc rational expression $R$, the realization that we have constructed in Theorem 2.4- centred at a $d$-tuple of $s \times s$ matrices - allows us to evaluate $R$ at every point in its domain of regularity and not only at the points whose dimension is a multiple of $s$. An alternative way to evaluate a nc rational expression on all of its domain of regularity, will be given later in Theorem 3.3. We define $P_{1}=E(n, s)$ and $P_{2}=E(n, L)$, correspondingly to (1.1).

Proposition 2.5. Let $n \geq 1, \mathcal{A}_{n}=\mathbb{K}^{n \times n}, \mathcal{R}$ be a nc Fornasini-Marchesini realization centred at $\underline{Y} \in\left(\mathbb{K}^{s \times s}\right)^{d}$ and $\underline{X} \in\left(\mathbb{K}^{s n \times s n}\right)^{d}$. Then

$$
\underline{X} \in D O M^{\mathcal{A}_{n}}(\mathcal{R}) \Longleftrightarrow P_{1} \cdot \underline{X} \cdot P_{1}^{-1} \in D O M_{s n}(\mathcal{R})
$$

and $\mathcal{R}^{\mathcal{A}_{n}}(\underline{X})=P_{1}^{-1} \mathcal{R}\left(P_{1} \cdot \underline{X} \cdot P_{1}^{-1}\right) P_{1}$, whenever $(2.9)$ holds. 
Proof. Let $\underline{X}=\left(X_{1}, \ldots, X_{d}\right) \in\left(\mathbb{K}^{s n \times s n}\right)^{d}$ and consider the decomposition (2.2), where $X_{i j}^{(k)} \in \mathbb{K}^{n \times n}=\mathcal{A}_{n}$ for $1 \leq i, j \leq s$ and $1 \leq k \leq d$. As

$$
\begin{gathered}
I_{L} \otimes 1_{\mathcal{A}}-\sum_{k=1}^{d}\left(X_{k}-Y_{k} \otimes 1_{\mathcal{A}}\right) \mathbf{A}_{k}^{\mathcal{A}_{n}}=I_{L n}-\sum_{k=1}^{d}\left[\sum_{i, j=1}^{s} \mathbf{A}_{k}\left(E_{i j}\right) \otimes X_{i j}^{(k)}-\mathbf{A}_{k}\left(Y_{k}\right) \otimes I_{n}\right]= \\
P_{2}^{-1}\left(I_{L n}-\sum_{k=1}^{d}\left[\sum_{i, j=1}^{s} X_{i j}^{(k)} \otimes \mathbf{A}_{k}\left(E_{i j}\right)-I_{n} \otimes \mathbf{A}_{k}\left(Y_{k}\right)\right]\right) P_{2}=P_{2}^{-1}\left(I_{n} \otimes I_{L}-\sum_{k=1}^{d}\left[\sum_{i, j=1}^{s}\right.\right. \\
\left.\left.\left(X_{i j}^{(k)} \otimes E_{i j}\right) \mathbf{A}_{k}-\left(I_{n} \otimes Y_{k}\right) \mathbf{A}_{k}\right]\right) P_{2}=P_{2}^{-1}\left(I_{n} \otimes I_{L}-\sum_{k=1}^{d}\left(P_{1} X_{k} P_{1}^{-1}-I_{n} \otimes Y_{k}\right) \mathbf{A}_{k}\right) P_{2},
\end{gathered}
$$

we have $\underline{X} \in D O M^{\mathcal{A}_{n}}(\mathcal{R})$ if and only if $P_{1} \cdot \underline{X} \cdot P_{1}^{-1} \in D O M_{s n}(\mathcal{R})$.

Similar computation shows that

$$
\begin{aligned}
\left(X_{k}\right) \mathbf{B}_{k}^{\mathcal{A}_{n}}-\mathbf{B}_{k}\left(Y_{k}\right) \otimes I_{n}= & \sum_{i, j=1}^{s} \mathbf{B}_{k}\left(E_{i j}\right) \otimes X_{i j}^{(k)}-\mathbf{B}_{k}\left(Y_{k}\right) \otimes I_{n}=P_{2}^{-1}\left(\sum_{i, j=1}^{s} X_{i j}^{(k)} \otimes \mathbf{B}_{k}\left(E_{i j}\right)\right. \\
& \left.-I_{n} \otimes \mathbf{B}_{k}\left(Y_{k}\right)\right) P_{1}=P_{2}^{-1}\left(P_{1} X_{k} P_{1}^{-1}-I_{n} \otimes Y_{k}\right) \mathbf{B}_{k} P_{1}
\end{aligned}
$$

and hence $\underline{X} \in D O M^{\mathcal{A}_{n}}(\mathcal{R})$ implies

$$
\begin{gathered}
\mathcal{R}^{\mathcal{A}_{n}}(\underline{X})=D \otimes I_{n}+\left(C \otimes I_{n}\right)\left(I_{L n}-\sum_{k=1}^{d}\left[\left(X_{k}\right) \mathbf{A}_{k}^{\mathcal{A}_{n}}-\mathbf{A}_{k}\left(Y_{k}\right) \otimes I_{n}\right]\right)^{-1} \sum_{k=1}^{d}\left[\left(X_{k}\right) \mathbf{B}_{k}^{\mathcal{A}_{n}}-\right. \\
\left.\mathbf{B}_{k}\left(Y_{k}\right) \otimes I_{n}\right]=D \otimes I_{n}+\left(C \otimes I_{n}\right) P_{2}^{-1}\left(I_{n} \otimes I_{L}-\sum_{k=1}^{d}\left(P_{1} X_{k} P_{1}^{-1}-I_{n} \otimes Y_{k}\right) \mathbf{A}_{k}\right)^{-1} \sum_{k=1}^{d}\left(P_{1} X_{k} P_{1}^{-1}\right. \\
\left.-I_{n} \otimes Y_{k}\right) \mathbf{B}_{k} P_{1}=P_{1}^{-1} \mathcal{R}\left(P_{1} \cdot \underline{X} \cdot P_{1}^{-1}\right) P_{1} .
\end{gathered}
$$

Corollary 2.6. If $n \in \mathbb{N}$ and $\mathcal{R}$ is a nc Fornasini-Marchesini realization of a $n c$ rational expression $R$ w.r.t $\mathcal{A}_{n}=\mathbb{K}^{n \times n}$, then for every $\underline{X} \in \operatorname{dom}_{n}(R)$ we have

$$
\underline{X} \otimes I_{s} \in D O M_{s n}(\mathcal{R}) \text { and } R(\underline{X}) \otimes I_{s}=\mathcal{R}\left(\underline{X} \otimes I_{s}\right) .
$$

Proof. Let $\underline{X} \in \operatorname{dom}_{n}(R)$, thus $\underline{X} \in \operatorname{dom}^{\mathcal{A}_{n}}(R)$ and that implies by Definition 2.3 that $I_{s} \otimes \underline{X} \in D O M^{\mathcal{A}_{n}}(\mathcal{R})$ and $\mathcal{R}^{\mathcal{A}_{n}}\left(I_{s} \otimes \underline{X}\right)=I_{s} \otimes R^{\mathcal{A}_{n}}(\underline{X})$. Using Proposition 2.5 , we get

$$
\underline{X} \otimes I_{s}=P_{1} \cdot\left(I_{s} \otimes \underline{X}\right) \cdot P_{1}^{-1} \in D O M_{s n}(\mathcal{R})
$$

and

$$
P_{1}^{-1} \mathcal{R}\left(\underline{X} \otimes I_{s}\right) P_{1}=\mathcal{R}^{\mathcal{A}_{n}}\left(I_{s} \otimes \underline{X}\right)=I_{s} \otimes R(\underline{X})
$$

which implies that $\mathcal{R}\left(\underline{X} \otimes I_{s}\right)=R(\underline{X}) \otimes I_{s}$.

2.2. Controllability and observability. To consider the notion of minimal realizations as in the classical realization theory, we first introduce the definitions of controllability and observability in the case of nc Fornasini-Marchesini realizations centred at a matrix point, which are generalizations of the definitions in the case of nc Fornasini-Marchesini realizations centred at a scalar point. For a reference of the later ones see [8]. 
Given the linear mappings $\mathbf{A}_{1}, \ldots, \mathbf{A}_{d}: \mathbb{K}^{s \times s} \rightarrow \mathbb{K}^{L \times L}, \mathbf{B}_{k}: \mathbb{K}^{s \times s} \rightarrow \mathbb{K}^{L \times s}$ and a word $\omega=g_{i_{1}} \ldots g_{i_{\ell}} \in \mathcal{G}_{d}$ of length $|\omega|=\ell$, define the multilinear mapping $\underline{\mathbf{A}}^{\omega}:\left(\mathbb{K}^{s \times s}\right)^{\ell} \rightarrow \mathbb{K}^{L \times L}$ by

$$
\underline{\mathbf{A}}^{\omega}\left(X_{1}, \ldots, X_{\ell}\right):=\mathbf{A}_{i_{1}}\left(X_{1}\right) \cdots \mathbf{A}_{i_{\ell}}\left(X_{\ell}\right)
$$

and the multilinear mapping $\underline{\mathbf{A}}^{\omega} \cdot \mathbf{B}_{k}:\left(\mathbb{K}^{s \times s}\right)^{\ell+1} \rightarrow \mathbb{K}^{L \times s}$ by

$$
\left(\underline{\mathbf{A}}^{\omega} \cdot \mathbf{B}_{k}\right)\left(X_{1}, \ldots, X_{\ell+1}\right):=\underline{\mathbf{A}}^{\omega}\left(X_{1}, \ldots, X_{\ell}\right) \mathbf{B}_{k}\left(X_{\ell+1}\right) .
$$

Definition 2.7. Let $\mathbf{A}_{1}, \ldots, \mathbf{A}_{d}: \mathbb{K}^{s \times s} \rightarrow \mathbb{K}^{L \times L}$ and $\mathbf{B}_{1}, \ldots, \mathbf{B}_{d}: \mathbb{K}^{s \times s} \rightarrow \mathbb{K}^{L \times s}$ be linear mappings, and $C \in \mathbb{K}^{s \times L}$.

1. The controllable subspace $\mathcal{C}_{\underline{\mathbf{A}}, \underline{\mathrm{B}}}$ is defined by

$$
\bigvee_{\omega \in \mathcal{G}_{d}, X_{1}, \ldots, X_{|\omega|+1} \in \mathbb{K}^{s \times s}, 1 \leq k \leq d} \operatorname{Im}\left(\underline{\mathbf{A}}^{\omega}\left(X_{1}, \ldots, X_{|\omega|}\right) \mathbf{B}_{k}\left(X_{|\omega|+1}\right)\right) .
$$

If $\mathcal{C}_{\underline{\mathbf{A}}, \underline{\mathbf{B}}}=\mathbb{K}^{L}$, then the tuple $(\underline{\mathbf{A}}, \underline{\mathbf{B}})$ is called controllable.

2. The un-observable subspace $\mathcal{N O}_{C, \underline{\mathbf{A}}}$ is defined by

$$
\bigcap_{\omega \in \mathcal{G}_{d}, X_{1}, \ldots, X_{|\omega|} \in \mathbb{K}^{s \times s}} \operatorname{ker}\left(C \underline{\mathbf{A}}^{\omega}\left(X_{1}, \ldots, X_{|\omega|}\right)\right) .
$$

$$
\text { If } \mathcal{N} \mathcal{O}_{C, \underline{\mathbf{A}}}=\{\underline{0}\} \text {, the tuple }(C, \underline{\mathbf{A}}) \text { is called observable. }
$$

The multilinear mapping $\underline{\mathbf{A}}^{\omega}$ can be viewed as a linear mapping from $\left(\mathbb{K}^{s \times s}\right)^{\ell}$ to $\mathbb{K}^{L \times L}$. Then one can use the faux product, as introduced in (1.2), to define controllability and observability not only on the level of $s \times s$ matrices, but also on the levels of $s m \times s m$ matrices, for every $m \in \mathbb{N}$, using the subspaces

$$
\mathcal{C}_{\underline{\mathbf{A}}, \underline{\mathbf{B}}}^{(m)}=\bigvee_{\omega \in \mathcal{G}_{d}, X_{1}, \ldots, X_{|\omega|+1} \in \mathbb{K}^{s m \times s m}, 1 \leq k \leq d} \operatorname{Im}\left(\left(X_{1} \odot_{s} \cdots \odot_{s} X_{|\omega|}\right) \underline{\mathbf{A}}^{\omega}\left(X_{|\omega|+1}\right) \mathbf{B}_{k}\right)
$$

and

$$
\mathcal{N} \mathcal{O}_{C, \underline{\mathbf{A}}}^{(m)}=\bigcap_{\omega \in \mathcal{G}_{d}, X_{1}, \ldots, X_{|\omega|} \in \mathbb{K}^{s m \times s m}} \operatorname{ker}\left(\left(I_{m} \otimes C\right)\left(X_{1} \odot_{s} \cdots \odot_{s} X_{|\omega|}\right) \underline{\mathbf{A}}^{\omega}\right) .
$$

Proposition 2.8. If $m \in \mathbb{N}$, then $\mathcal{C}_{\underline{\mathbf{A}}, \underline{\mathbf{B}}}^{(m)}=\mathcal{C}_{\underline{\mathbf{A}}, \underline{\mathbf{B}}}^{(1)} \otimes \mathbb{K}^{m}$ and $\mathcal{N} \mathcal{O}_{C, \underline{\mathbf{A}}}^{(m)}=\mathcal{N} \mathcal{O}_{C, \underline{\mathbf{A}}}^{(1)} \otimes \mathbb{K}^{m}$.

Proof. Let $m \in \mathbb{N}$.

- If $\underline{u} \in \mathcal{C}_{\underline{\mathbf{A}}, \underline{\mathbf{B}}}^{(m)}$, then $\underline{u}$ is a linear combination of vectors of the form

$$
\left(X_{1}\right) \mathbf{A}_{i_{1}} \cdots\left(X_{k}\right) \mathbf{A}_{i_{k}}\left(X_{k+1}\right) \mathbf{B}_{i_{k+1}} \underline{u}_{i},
$$

where $1 \leq i_{1}, \ldots, i_{k+1} \leq d, X_{1}, \ldots, X_{k+1} \in \mathbb{K}^{s m \times s m}$ and $\underline{u}_{i} \in \mathbb{K}^{s m}$. As the mappings $\mathbf{A}_{i}, \mathbf{B}_{i}(1 \leq i \leq d)$ act on $s m \times s m$ matrices by acting on their $s \times s$ blocks, we get that $\left(X_{1}\right) \mathbf{A}_{i_{1}} \cdots\left(X_{k}\right) \mathbf{A}_{i_{k}}\left(X_{k+1}\right) \mathbf{B}_{i_{k+1}} \underline{u}_{i} \in \mathcal{C}_{\underline{\mathbf{A}}, \underline{B}}^{(1)} \otimes \mathbb{K}^{m}$ and as a linear combination of such vectors, we get that $\underline{u} \in \mathcal{C}_{\underline{\mathbf{A}}, \mathbf{B}}^{(1)} \otimes \mathbb{K}^{m}$. 
- On the other hand, let $\underline{u} \in \mathcal{C}_{\underline{\mathbf{A}}, \underline{\mathbf{B}}}^{(1)} \otimes \mathbb{K}^{m}$ and write $\underline{u}=\left[\begin{array}{lll}\underline{u}_{1}^{T} & \cdots & \underline{u}_{m}^{T}\end{array}\right]^{T}$ where $\underline{u}_{1}, \ldots, \underline{u}_{m} \in \mathcal{C}_{\underline{\mathbf{A}}, \underline{\mathbf{B}}}^{(1)}$. Thus

$$
\begin{gathered}
\underline{u}=\sum_{i=1}^{m} \underline{u}_{i} \otimes \underline{e}_{i}=\sum_{i=1}^{m}\left(\sum_{j=1}^{k_{i}} \underline{\mathbf{A}}^{\omega_{j, i}}\left(X_{j, 1}^{(i)}, \ldots, X_{\left.j, \mid \omega_{j, i}\right)}^{(i)}\right) \mathbf{B}_{\ell_{i, j}}\left(\widetilde{X}_{j}^{(i)}\right) \underline{w}_{j, i}\right) \otimes \underline{e}_{i}=\sum_{i=1}^{m} \sum_{j=1}^{k_{i}} \\
{\left[I_{m} \otimes\left(\underline{\mathbf{A}}^{\omega_{j, i}}\left(X_{j, 1}^{(i)}, \ldots, X_{j,\left|\omega_{j, i}\right|}^{(i)}\right) \mathbf{B}_{\ell_{i, j}}\left(\widetilde{X}_{j}^{(i)}\right) \underline{w}_{j, i}\right)\right] \underline{e}_{i}=\sum_{i=1}^{m} \sum_{j=1}^{k_{i}}\left(\left(I_{m} \otimes X_{j, 1}^{(i)}\right) \odot_{s} \ldots\right.} \\
\left.\odot_{s}\left(I_{m} \otimes X_{j,\left|\omega_{j, i}\right|}^{(i)}\right)\right) \underline{\mathbf{A}}^{\omega_{j, i}}\left(I_{m} \otimes \widetilde{X}_{j}^{(i)}\right) \mathbf{B}_{\ell_{i, j}}\left(I_{m} \otimes \underline{w}_{j, i}\right) \underline{e}_{i} \in \mathcal{C}_{\underline{\mathbf{A}}, \underline{\mathbf{B}}}^{(m)},
\end{gathered}
$$

where $\underline{e}_{1}, \ldots, \underline{e}_{m}$ is the standard basis of $\mathbb{K}^{m}, k_{i} \in \mathbb{N}, \omega_{j, i} \in \mathcal{G}_{d}, 1 \leq \ell_{i, j} \leq d$ and $X_{j, 1}^{(i)}, \ldots, X_{j,\left|\omega_{j, i}\right|}^{(i)}, \widetilde{X}_{j}^{(i)} \in \mathbb{K}^{s \times s}$ for $1 \leq j \leq k_{i}$ and $1 \leq i \leq m$.

- Next, let

$$
\underline{u}=\left[\begin{array}{lll}
\underline{u}_{1}^{T} & \cdots & \underline{u}_{m}^{T}
\end{array}\right]^{T} \in \mathcal{N} \mathcal{O}_{C, \underline{\mathbf{A}}}^{(m)},
$$

then for all $\omega \in \mathcal{G}_{d}, 1 \leq j \leq m$ and $X_{1}, \ldots, X_{|\omega|} \in \mathbb{K}^{s \times s}$, we have

$$
\begin{aligned}
\underline{0}=\left(I_{m} \otimes C\right) & \left(\left(E_{j j} \otimes X_{1}\right) \odot_{s} \cdots \odot_{s}\left(E_{j j} \otimes X_{|\omega|}\right)\right) \underline{\mathbf{A}}^{\omega} \underline{u} \\
& =\left(I_{m} \otimes C\right)\left(E_{j j} \otimes \underline{\mathbf{A}}^{\omega}\left(X_{1}, \ldots, X_{|\omega|}\right)\right) \underline{u}=C \underline{\mathbf{A}}^{\omega}\left(X_{1}, \ldots, X_{|\omega|}\right) \underline{u}_{j},
\end{aligned}
$$

i.e., $\underline{u}_{j} \in \mathcal{N} \mathcal{O}_{C, \underline{\mathbf{A}}}^{(1)}$ and hence $\underline{u} \in \mathcal{N} \mathcal{O}_{C, \underline{\mathbf{A}}}^{(1)} \otimes \mathbb{K}^{m}$.

- On the other hand, let

$$
\underline{u}=\left[\begin{array}{lll}
\underline{u}_{1}^{T} & \cdots & \underline{u}_{m}^{T}
\end{array}\right]^{T} \in \mathcal{N} \mathcal{O}_{C, \underline{\mathbf{A}}}^{(1)} \otimes \mathbb{K}^{m},
$$

then for every $\omega=g_{i_{1}} \ldots g_{i_{k}} \in \mathcal{G}_{d}$ and $Z_{1}, \ldots, Z_{k} \in \mathbb{K}^{s m \times s m}$ we have

$$
\begin{aligned}
\left(I_{m} \otimes C\right)\left(Z_{1} \odot_{s} \cdots \odot_{s} Z_{|\omega|}\right) \underline{\mathbf{A}}^{\omega} \underline{u}=\left(I_{m} \otimes C\right)\left(Z_{1}\right) \mathbf{A}_{i_{1}} \cdots\left(Z_{k}\right) \mathbf{A}_{i_{k}} \underline{u}= \\
\left(I_{m} \otimes C\right)\left[\mathbf{A}_{i_{1}}\left(Z_{p, q}^{(1)}\right)\right]_{1 \leq p, q \leq m} \cdots\left[\mathbf{A}_{i_{k}}\left(Z_{p, q}^{(k)}\right)\right]_{1 \leq p, q \leq m}\left[\begin{array}{c}
\underline{u}_{1} \\
\vdots \\
\underline{u}_{m}
\end{array}\right]=\underline{0}
\end{aligned}
$$

as $\underline{u}_{1}, \ldots, \underline{u}_{m} \in \mathcal{N} \mathcal{O}_{C, \underline{\mathbf{A}}}^{(1)}$ and each of the entries in the product is a linear combination of vectors of the form $C \mathbf{A}_{i_{1}}\left(Z_{p_{1}, q_{1}}^{(1)}\right) \cdots \mathbf{A}_{i_{k}}\left(Z_{p_{k}, q_{k}}^{(k)}\right) \underline{u}_{i}$ where $1 \leq i \leq m$, which are all $\underline{0}$.

Two immediate consequences of Proposition 2.8 are the following. If $(\underline{\mathbf{A}}, \underline{\mathbf{B}})$ is controllable, then $\mathcal{C}_{\underline{\mathbf{A}}, \underline{\mathbf{B}}}^{(m)}=\mathbb{K}^{L m}$ for all $m \in \mathbb{N}$; whereas if $\mathcal{C}_{\underline{\mathbf{A}}, \underline{\mathbf{B}}}^{(m)}=\mathbb{K}^{L m}$ for some $m \in \mathbb{N}$, then $(\underline{\mathbf{A}}, \underline{\mathbf{B}})$ is controllable. Similarly, if $(C, \underline{\mathbf{A}})$ is observable, then $\mathcal{N} \mathcal{O}_{C, \underline{\mathbf{A}}}^{(m)}=\{\underline{0}\}$ for all $m \in \mathbb{N}$; whereas if $\mathcal{N} \mathcal{O}_{C, \underline{\mathbf{A}}}^{(m)}=\{\underline{0}\}$ for some $m \in \mathbb{N}$, then $(C, \underline{\mathbf{A}})$ is observable

Next, we show how the original definitions of controllability and observability may be reformulated using the standard basis $\mathcal{E}_{s}$ of $\mathbb{K}^{s \times s}$.

Proposition 2.9. Let $\mathbf{A}_{1}, \ldots, \mathbf{A}_{d}: \mathbb{K}^{s \times s} \rightarrow \mathbb{K}^{L \times L}$ and $\mathbf{B}_{1}, \ldots, \mathbf{B}_{d}: \mathbb{K}^{s \times s} \rightarrow \mathbb{K}^{L \times s}$ be linear mappings, and $C \in \mathbb{K}^{s \times L}$. Then 
1. $(\underline{\mathbf{A}}, \underline{\mathbf{B}})$ is controllable if and only if

$$
\bigvee_{\omega \in \mathcal{G}_{d}, X_{1}, \ldots, X_{|\omega|+1} \in \mathcal{E}_{s}, 1 \leq k \leq d} \operatorname{Im}\left(\underline{\mathbf{A}}^{\omega}\left(X_{1}, \ldots, X_{|\omega|}\right) \mathbf{B}_{k}\left(X_{|\omega|+1}\right)\right)=\mathbb{K}^{L} .
$$

2. $(C, \underline{\mathbf{A}})$ is observable if and only if

$$
\bigcap_{\omega \in \mathcal{G}_{d}, X_{1}, \ldots, X_{|\omega|} \in \mathcal{E}_{s}} \operatorname{ker}\left(C \underline{\mathbf{A}}^{\omega}\left(X_{1}, \ldots, X_{|\omega|}\right)\right)=\{\underline{0}\} .
$$

Proof. Since $\mathcal{E}_{s} \subseteq \mathbb{K}^{s \times s}$ the direction $\Longleftarrow$ of part $\mathbf{1}$ is trivial. To prove the other direction, suppose $(\underline{\mathbf{A}}, \underline{\mathbf{B}})$ is controllable, let $X_{1}, \ldots, X_{\ell+1} \in \mathbb{K}^{s \times s}$ and $\omega=$ $g_{j_{1}} \ldots g_{j_{\ell}} \in \mathcal{G}_{d}$. Thus, one can write $X_{t}=\sum_{p, q=1}^{s} E_{p q} \otimes x_{p q}^{(t)}$ for $1 \leq t \leq \ell+1$ and by linearity of $\mathbf{A}_{k}, \mathbf{B}_{k}$ we get

$$
\begin{array}{r}
\underline{\mathbf{A}}^{\omega}\left(X_{1}, \ldots, X_{\ell}\right) \mathbf{B}_{k}\left(X_{\ell+1}\right)=\left(\prod_{t=1}^{\ell} \sum_{p_{t}, q_{t}=1}^{s} x_{p_{t}, q_{t}}^{(t)} \mathbf{A}_{j_{t}}\left(E_{p_{t} q_{t}}\right)\right) \sum_{p, q=1}^{s} x_{p, q}^{(t+1)} \mathbf{B}_{k}\left(E_{p q}\right) \\
\in \bigvee_{\nu \in \mathcal{G}_{d}, Z_{1}, \ldots, Z_{\ell+1} \in \mathcal{E}_{s}, 1 \leq k \leq d} \operatorname{Im}\left(\underline{\mathbf{A}}^{\nu}\left(Z_{1}, \ldots, Z_{\ell}\right) \mathbf{B}_{k}\left(Z_{\ell+1}\right)\right) .
\end{array}
$$

Therefore $\mathcal{C}_{\underline{\mathbf{A}}, \underline{\mathbf{B}}} \subseteq \bigvee_{\nu \in \mathcal{G}_{d}, Z_{1}, \ldots, Z_{\ell+1} \in \mathcal{E}_{s}, 1 \leq k \leq d} \operatorname{Im}\left(\underline{\mathbf{A}}^{\nu}\left(Z_{1}, \ldots, Z_{\ell}\right) \mathbf{B}_{k}\left(Z_{\ell+1}\right)\right)$ whereas $\mathcal{C}_{\underline{\mathbf{A}}, \underline{\mathbf{B}}}=\mathbb{K}^{L}$ implies the wanted equality. Similar proof holds for part $\mathbf{2}$.

The last part of this subsection discusses observability and controllability matrices. The infinite block matrix

$$
\mathfrak{C}_{\underline{\mathbf{A}}, \underline{\mathbf{B}}}:=\operatorname{row}\left[\mathfrak{C}_{\underline{\mathbf{A}}, \underline{\mathbf{B}}}^{(\omega, k)}\right]_{(\omega, k) \in \mathcal{G}_{d} \times\{1, \ldots, d\}}
$$

is called the controllability matrix associated with the tuple $(\underline{\mathbf{A}}, \underline{\mathbf{B}})$, where

$$
\mathfrak{C}_{\underline{\mathbf{A}}, \underline{\mathbf{B}}}^{(\omega, k)} \in \mathbb{K}^{L \times s^{3}(|\omega|+1)} \text { is given by }{\underline{\mathfrak{C}_{\underline{\mathbf{A}}}^{(\omega,}, \underline{\mathbf{B}}}}^{(\omega)}:=\operatorname{row}\left[\left(\underline{\mathbf{A}}^{\omega} \cdot \mathbf{B}_{k}\right)(\underline{Z})\right]_{\underline{Z} \in \mathcal{E}_{s}^{|\omega|+1}}
$$

for each $(\omega, k) \in \mathcal{G}_{d} \times\{1, \ldots, d\}$ and the infinite block matrix

$$
\mathfrak{O}_{C, \underline{\mathbf{A}}}:=\operatorname{col}\left[\mathfrak{O}_{C, \underline{\mathbf{A}}}^{(\omega)}\right]_{\omega \in \mathcal{G}_{d}}
$$

is called the observability matrix associated to the tuple $(C, \underline{\mathbf{A}})$, where

$$
\mathfrak{O}_{C, \underline{\mathbf{A}}}^{(\omega)} \in \mathbb{K}^{s^{3}|\omega| \times L} \text { is given by } \mathfrak{O}_{C, \underline{\mathbf{A}}}^{(\omega)}:=\operatorname{col}\left[C \cdot \underline{\mathbf{A}}^{\omega}(\underline{Z})\right]_{\underline{Z} \in \mathcal{E}_{s}^{|\omega|}}
$$

for each $\omega \in \mathcal{G}_{d}$. The following is a characterization of controllability and observability using the controllability and observability matrices. Most of the arguments in the proof are taken from linear algebra.

Proposition 2.10. Let $\mathbf{A}_{1}, \ldots, \mathbf{A}_{d}: \mathbb{K}^{s \times s} \rightarrow \mathbb{K}^{L \times L}$ and $\mathbf{B}_{1}, \ldots, \mathbf{B}_{d}: \mathbb{K}^{s \times s} \rightarrow$ $\mathbb{K}^{L \times s}$ be linear mappings, and $C \in \mathbb{K}^{s \times L}$. The following are equivalent:

1. $(\underline{\mathbf{A}}, \underline{\mathbf{B}})$ is controllable $[$ resp., $(C, \underline{\mathbf{A}})$ is observable $]$.

2. The matrix $\mathfrak{C}_{\underline{\mathbf{A}}, \underline{\mathbf{B}}}\left[\right.$ resp., $\left.\mathfrak{O}_{C, \underline{\mathbf{A}}}\right]$ is right $[$ resp., left $]$ invertible.

3. The finite block matrix row $\left[\mathfrak{C}_{\underline{\mathbf{A}}, \underline{\mathbf{B}}}^{(\omega, k)}\right]_{|\omega| \leq \ell, 1 \leq k \leq d}\left[\operatorname{resp}\right.$., col $\left.\left[\mathfrak{D}_{C, \underline{\mathbf{A}}}^{(\omega)}\right]_{|\omega| \leq \ell}\right]$ is right $[$ resp., left $]$ invertible for some $\ell \in \mathbb{N}$.

In that case, we can choose $\ell \leq L-1$. 


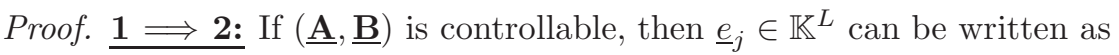

$$
\underline{e}_{j}=\sum_{i=1}^{k_{j}} \underline{\mathbf{A}}^{\omega_{j, i}}\left(X_{i, 1}^{(j)}, \ldots, X_{i,\left|\omega_{j, i}\right|}^{(j)}\right) \mathbf{B}_{\ell_{j, i}}\left(X_{i,\left|\omega_{j, i}\right|+1}^{(j)}\right) \underline{u}_{j, i}
$$

where $k_{j} \in \mathbb{N}, \omega_{j, i} \in \mathcal{G}_{d}, X_{i, 1}^{(j)}, \ldots, X_{i,\left|\omega_{j, i}\right|+1}^{(j)} \in \mathbb{K}^{s \times s}$ and $1 \leq \ell_{j, i} \leq d$, for every $1 \leq i \leq k_{j}$ and $1 \leq j \leq L$. Thus $\underline{e}_{1}, \ldots, \underline{e}_{L}$ belong to the column span of the matrix $\mathfrak{C}_{\underline{\mathbf{A}}, \underline{\underline{B}}}$ and hence $\mathfrak{C}_{\underline{\mathbf{A}}, \underline{B}}$ is right invertible.

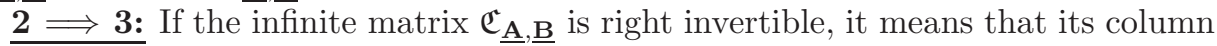
span is equal to $\mathbb{K}^{L}$, however this span of infinitely many vectors of length $L$ must coincide with a span of finitely many of the columns, which easily implies part $\mathbf{3}$.

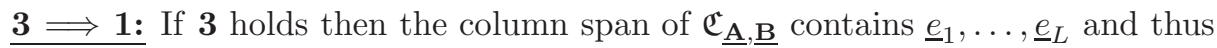
is equal to $\mathbb{K}^{L}$, i.e., $\mathcal{C}_{\underline{\mathbf{A}}, \underline{\mathbf{B}}}=\mathbb{K}^{L}$ and the tuple $(\underline{\mathbf{A}}, \underline{\mathbf{B}})$ is controllable.

- Suppose next that $(\underline{\mathbf{A}}, \underline{\mathbf{B}})$ is controllable, and define

$$
\mathcal{C}_{\ell}:=\bigvee_{|\omega| \leq \ell, 1 \leq k \leq d} \operatorname{Im}\left(\mathfrak{C}_{\underline{\mathbf{A}}, \underline{\mathbf{B}}}^{(\omega, k)}\right)=\bigvee_{|\omega| \leq \ell, 1 \leq k \leq d} \operatorname{Im}\left(\underline{\mathbf{A}}^{\omega} \cdot \mathbf{B}_{k}\right)
$$

for $\ell \geq 0$. Then $\mathcal{C}_{0} \subseteq \mathcal{C}_{1} \subseteq \ldots \subseteq \mathcal{C}_{\ell} \subseteq \mathcal{C}_{\ell+1} \subseteq \ldots$ are all subspaces of $\mathbb{K}^{L}$, whereas the controllability of $(\underline{\mathbf{A}}, \underline{\mathbf{B}})$ implies that $\bigcup_{\ell=0}^{\infty} \mathcal{C}_{\ell}=\mathbb{K}^{L}$ and $\mathcal{C}_{0} \neq\{0\}$. Moreover, it is easily seen that if $\mathcal{C}_{\ell_{0}}=\mathcal{C}_{\ell_{0}+1}$ for some $\ell_{0} \geq 0$, then $\mathcal{C}_{\ell_{0}+k}=\mathcal{C}_{\ell_{0}}$ for all $k \geq 0$ and hence

$$
\mathbb{K}^{L}=\bigcup_{\ell=0}^{\infty} \mathcal{C}_{\ell}=\mathcal{C}_{0} \cup \ldots \cup \mathcal{C}_{\ell_{0}}=\mathcal{C}_{\ell_{0}} .
$$

Therefore, the sequence $1 \leq \operatorname{dim}\left(\mathcal{C}_{0}\right) \leq \operatorname{dim}\left(\mathcal{C}_{1}\right) \leq \ldots \leq \operatorname{dim}\left(\mathcal{C}_{L}\right) \leq \ldots \leq L$ must coincide after at most $L-1$ inequalities, i.e., $\operatorname{dim}\left(\mathcal{C}_{L-1}\right)=L$ which means that $\mathbb{K}^{L}=\mathcal{C}_{\underline{\mathrm{A}}, \underline{\mathrm{B}}}=\mathcal{C}_{L-1}$.

- As for observability, to prove that $\mathbf{1} \Longrightarrow \mathbf{2} \Longrightarrow \mathbf{3} \Longrightarrow \mathbf{1}$ we use the same arguments as above; so we only show how to get the bound on the size of the matrix. Suppose that $(C, \underline{\mathbf{A}})$ is observable and define

$$
\mathcal{N O} \mathcal{O}_{\ell}:=\bigcap_{|\omega| \leq \ell, \underline{X} \in\left(\mathbb{K}^{s \times s}\right)^{|\omega|}} \operatorname{ker}\left(C \underline{\mathbf{A}}^{\omega}(\underline{X})\right)
$$

for $\ell \geq 0$. Then $\mathcal{N O}_{0} \supseteq \mathcal{N} \mathcal{O}_{1} \supseteq \ldots \supseteq \mathcal{N} \mathcal{O}_{\ell} \supseteq \mathcal{N} \mathcal{O}_{\ell+1} \supseteq \ldots$ are all subspaces of $\mathbb{K}^{L}$, whereas the observability of $(C, \underline{\mathbf{A}})$ implies that $\bigcap_{\ell \geq 0} \mathcal{N O}_{\ell}=\{\underline{0}\}$ and $\mathcal{N} \mathcal{O}_{0} \neq \mathbb{K}^{L}$. It is easily seen that if $\mathcal{N} \mathcal{O}_{\ell_{0}}=\mathcal{N} \mathcal{O}_{\ell_{0}+1}$ for some $\ell_{0} \geq 0$, then $\mathcal{N} \mathcal{O}_{\ell_{0}+k}=\mathcal{N} \mathcal{O}_{\ell_{0}}$ for all $k \geq 0$ and hence

$$
\{\underline{0}\}=\bigcap_{\ell \geq 0} \mathcal{N} \mathcal{O}_{\ell}=\mathcal{N} \mathcal{O}_{\ell_{0}} .
$$

Therefore the sequence $L>\operatorname{dim}\left(\mathcal{N O}_{0}\right) \geq \operatorname{dim}\left(\mathcal{N O}_{1}\right) \geq \ldots \geq \operatorname{dim}\left(\mathcal{N} \mathcal{O}_{L}\right) \geq \ldots$ must coincide after at most $L-1$ inequalities, i.e., $\mathcal{N O}_{L-1}=\{\underline{0}\}$.

2.3. Minimal realizations. A nc Fornasini-Marchesini realization of the form (2.1) is said to be

- controllable if the tuple $(\underline{\mathbf{A}}, \underline{\mathbf{B}})$ is controllable;

- observable if the tuple $(C, \underline{\mathbf{A}})$ is observable.

If $\mathcal{R}$ is a nc Fornasini-Marchesini realization of a nc rational expression $R$ centred at $\underline{Y}$, then it is said to be 
- minimal if the dimension $L$ is the smallest integer for which $R$ admits such a realization, i.e., if $\mathcal{R}^{\prime}$ is a nc Fornasini-Marchesini realization of $R$ centred at $\underline{Y}$ of dimension $L^{\prime}$, then $L \leq L^{\prime}$.

Remark 2.11. In fact, the minimality of a realization $\mathcal{R}$ is w.r.t rational functions, meaning that if $\mathcal{R}$ is a minimal nc Fornasini-Marchesini realization of $R$ centred at $\underline{Y}$, then it is also a minimal nc Fornasini-Marchesini realization of any nc rational expression $\widetilde{R}$ which is $\left(\mathbb{K}^{d}\right)_{n c}$-evaluation equivalent to $R$ (cf. Lemma 3.1).

We proceed by showing that every two controllable and observable nc FornasiniMarchesini realizations centred at $\underline{Y}$ of $\left(\mathbb{K}^{d}\right)_{n c}$-evaluation equivalent nc rational expressions must be similar, where most of the ideas of the proof are taken from $[7,13]$. We will also use the following facts, see [49, Theorem 4.8] and [47]:

- If $R$ is a nc rational expression and $\underline{Y} \in \operatorname{dom}_{s}(R)$, then

$\operatorname{Nilp}(\underline{Y} ; s m):=\left\{\underline{X} \in\left(\mathbb{K}^{s m \times s m}\right)^{d}: \underline{X}-I_{m} \otimes \underline{Y}\right.$ is jointly nilpotent $\} \subseteq \operatorname{dom}_{s m}(R)$

for every $m \in \mathbb{N}$, where a tuple $\underline{Z}=\left(Z_{1}, \ldots, Z_{d}\right) \in\left(\mathbb{K}^{s m \times s m}\right)^{d}$ is called jointly nilpotent if there exists $\kappa \in \mathbb{N}$ such that $\underline{Z}^{\odot{ }_{s} \omega}=0$ for all $\omega \in \mathcal{G}_{d}$ satisfying $|\omega| \geq \kappa$.

- $\left.R\right|_{N i l p(\underline{Y})}$ is a nc function on the nilpotent ball around $\underline{Y}$, that is

$$
N i l p(\underline{Y}):=\coprod_{m=1}^{\infty} N i l p(\underline{Y} ; s m) .
$$

- Every nc function on $N i l p(\underline{Y})$ has a power series expansion around $\underline{Y}$ of the form

$$
\sum_{\omega \in \mathcal{G}_{d}}\left(\underline{X}-I_{m} \otimes \underline{Y}\right)^{\odot_{s} \omega} \mathcal{R}_{\omega}, \underline{X} \in\left(\mathbb{K}^{s m \times s m}\right)^{d}
$$

where $\mathcal{R}_{\omega}$ are $|\omega|$-linear mappings from $\left(\mathbb{K}^{s \times s}\right)^{|\omega|}$ to $\mathbb{K}^{s \times s}$, called the Taylor-Taylor coefficients and are uniquely determined, see [47, Theorem 5.9]; notice that the sum is actually finite.

Lemma 2.12. If $R$ is a nc rational expression in $x_{1}, \ldots, x_{d}$ over $\mathbb{K}$ and $\mathcal{R}$ is a $n c$ Fornasini-Marchesini realization of $R$ centred at $\underline{Y} \in \operatorname{dom}_{s}(R)$, of the form (2.1), then the Taylor-Taylor coefficients of $R$ are given by $\mathcal{R}_{\emptyset}:=D$ and the multilinear mappings $\mathcal{R}_{\omega g_{k}}:=C \cdot \underline{\mathbf{A}}^{\omega} \cdot \mathbf{B}_{k}:\left(\mathbb{K}^{s \times s}\right)^{\ell+1} \rightarrow \mathbb{K}^{s \times s}$ which act as

$$
\mathcal{R}_{\omega g_{k}}\left(Z_{1}, \ldots, Z_{\ell+1}\right)=C \mathbf{A}_{i_{1}}\left(Z_{1}\right) \cdots \mathbf{A}_{i_{\ell}}\left(Z_{\ell}\right) \mathbf{B}_{k}\left(Z_{\ell+1}\right)
$$

for $Z_{1}, \ldots, Z_{\ell+1} \in \mathbb{K}^{s \times s}, \omega=g_{i_{1}} \ldots g_{i_{\ell}} \in \mathcal{G}_{d}$ and $1 \leq k \leq d$. Moreover, if $Z_{1}, \ldots, Z_{\ell+1} \in \mathbb{K}^{s m \times s m}$, then

$$
\left(Z_{1} \odot_{s} \cdots \odot_{s} Z_{\ell+1}\right) \mathcal{R}_{\omega g_{k}}=\left(I_{m} \otimes C\right)\left(Z_{1}\right) \mathbf{A}_{i_{1}} \cdots\left(Z_{\ell}\right) \mathbf{A}_{\ell}\left(Z_{\ell+1}\right) \mathbf{B}_{k} .
$$

Proof. Let $\underline{X} \in \operatorname{Nilp}(\underline{Y} ; s m)$, then we use the Neumann series w.r.t nilpotent elements in $\left(\mathbf{T}\left(\mathbb{K}^{s \times s}\right)\right)^{m \times m}$ - where $\mathbf{T}\left(\mathbb{K}^{s \times s}\right)$ is the tensor algebra of $\mathbb{K}^{s \times s}$ - to obtain that

$$
\left(I_{L m}-\sum_{k=1}^{d}\left(X_{k}-I_{m} \otimes Y_{k}\right) \mathbf{A}_{k}\right) \sum_{\omega \in \mathcal{G}_{d}}\left(\underline{X}-I_{m} \otimes \underline{Y}\right)^{\odot s \omega} \underline{\mathbf{A}}^{\omega}=I_{L m},
$$


where the second multiplicative term is actually a finite sum. As $\underline{Y} \in d_{0 m}(R)$ it follows that $N i l p(\underline{Y} ; s m) \subseteq \operatorname{dom}_{s m}(R)$, thus $\underline{X} \in \operatorname{dom}_{s m}(R) \subseteq D O M_{s m}(\mathcal{R})$ and

$$
\begin{aligned}
& R(\underline{X})=\mathcal{R}(\underline{X})=\left(I_{m} \otimes D\right)+\left(I_{m} \otimes C\right)\left(\sum_{\omega \in \mathcal{G}_{d}}\left(\underline{X}-I_{m} \otimes \underline{Y}\right)^{\odot_{s} \omega} \underline{\mathbf{A}}^{\omega}\right) \sum_{k=1}^{d}\left(X_{k}-I_{m} \otimes Y_{k}\right) \mathbf{B}_{k} \\
= & I_{m} \otimes D+\sum_{\omega \in \mathcal{G}_{d}, 1 \leq k \leq d}\left(\underline{X}-I_{m} \otimes \underline{Y}\right)^{\odot_{s}\left(\omega g_{k}\right)} \mathcal{R}_{\omega g_{k}}=\sum_{\nu \in \mathcal{G}_{d}}\left(\underline{X}-I_{m} \otimes \underline{Y}\right)^{\odot_{s} \nu} \mathcal{R}_{\nu},
\end{aligned}
$$

where the multilinear mappings $\left(\mathcal{R}_{\omega g_{k}}\right)$ are given by (2.12). However, $\left.R\right|_{N i l p(\underline{Y})}$ is a nc function on $\operatorname{Nilp}(\underline{Y})$ and so it has a unique Taylor-Taylor expansion, given by the coefficients $\left(\mathcal{R}_{\nu}\right)_{\nu \in \mathcal{G}_{d}}$.

Theorem 2.13 (Similarity of minimal realizations). Let $R_{1}$ and $R_{2}$ be two $n c$ rational expressions in $x_{1}, \ldots, x_{d}$ over $\mathbb{K}$, which admit nc Fornasini-Marchesini realizations

$$
\mathcal{R}_{1}(\underline{X})=D^{1}+C^{1}\left(I_{L_{1}}-\sum_{k=1}^{d} \mathbf{A}_{k}^{1}\left(X_{k}-Y_{k}\right)\right)^{-1} \sum_{k=1}^{d} \mathbf{B}_{k}^{1}\left(X_{k}-Y_{k}\right)
$$

and

$$
\mathcal{R}_{2}(\underline{X})=D^{2}+C^{2}\left(I_{L_{2}}-\sum_{k=1}^{d} \mathbf{A}_{k}^{2}\left(X_{k}-Y_{k}\right)\right)^{-1} \sum_{k=1}^{d} \mathbf{B}_{k}^{2}\left(X_{k}-Y_{k}\right),
$$

respectively, both centred at $\underline{Y} \in\left(\mathbb{K}^{s \times s}\right)^{d}$. Assume both $\mathcal{R}_{1}$ and $\mathcal{R}_{2}$ are controllable and observable.

If $R_{1}$ and $R_{2}$ are $\left(\mathbb{K}^{d}\right)_{n c}$-evaluation equivalent, then $\mathcal{R}_{1}$ and $\mathcal{R}_{2}$ are uniquely similar, i.e., $L_{1}=L_{2}, D^{1}=D^{2}$ and there exists a unique invertible matrix $T \in$ $\mathbb{K}^{L_{1} \times L_{1}}$ such that

$$
C^{2}=C^{1} T^{-1}, \mathbf{B}_{k}^{2}=T \cdot \mathbf{B}_{k}^{1} \text { and } \mathbf{A}_{k}^{2}=T \cdot \mathbf{A}_{k}^{1} \cdot T^{-1}, 1 \leq k \leq d .
$$

Moreover,

$$
D O M_{s m}\left(\mathcal{R}_{1}\right)=D O M_{s m}\left(\mathcal{R}_{2}\right) \text { and } \mathcal{R}_{1}(\underline{X})=\mathcal{R}_{2}(\underline{X}), \forall \underline{X} \in D O M_{s m}\left(\mathcal{R}_{1}\right)
$$

for every $m \in \mathbb{N}$, and for any unital $\mathbb{K}$-algebra $\mathcal{A}$ :

$$
D O M^{\mathcal{A}}\left(\mathcal{R}_{1}\right)=D O M^{\mathcal{A}}\left(\mathcal{R}_{2}\right) \text { and } \mathcal{R}_{1}^{\mathcal{A}}(\underline{\mathfrak{A}})=\mathcal{R}_{2}^{\mathcal{A}}(\underline{\mathfrak{A}}), \forall \underline{\mathfrak{A}} \in D O M^{\mathcal{A}}\left(\mathcal{R}_{1}\right) .
$$

Proof. From Lemma 2.12, the Taylor-Taylor coefficients of the nc rational expressions $R_{1}$ and $R_{2}$ (w.r.t the centre $\underline{Y}$ ) are

$$
\mathcal{R}_{\omega g_{k}}^{(1)}=C^{1} \cdot\left(\underline{\mathbf{A}}^{1}\right)^{\omega} \cdot \mathbf{B}_{k}^{1} \text { and } \mathcal{R}_{\omega g_{k}}^{(2)}=C^{2} \cdot\left(\underline{\mathbf{A}}^{2}\right)^{\omega} \cdot \mathbf{B}_{k}^{2},
$$

respectively. Since $R_{1}$ and $R_{2}$ are $\left(\mathbb{K}^{d}\right)_{n c}$-evaluation equivalent, their restrictions to $\operatorname{Nil}(\underline{Y})$ produce the same nc function and therefore, by the uniqueness of the Taylor-Taylor coefficients, $\mathcal{R}_{\emptyset}^{(1)}=\mathcal{R}_{\emptyset}^{(2)}$ and $\mathcal{R}_{\omega g_{k}}^{(1)}=\mathcal{R}_{\omega g_{k}}^{(2)}$ as multilinear mappings for every $\omega \in \mathcal{G}_{d}$ and $1 \leq k \leq d$, i.e., $D^{1}=D^{2}$ and

$$
C^{1} \cdot\left(\underline{\mathbf{A}}^{1}\right)^{\omega} \cdot \mathbf{B}_{k}^{1}=C^{2} \cdot\left(\underline{\mathbf{A}}^{2}\right)^{\omega} \cdot \mathbf{B}_{k}^{2} .
$$

Define a mapping $\mathcal{T}$ in the following way: for every $\omega \in \mathcal{G}_{d}, 1 \leq k \leq d, \underline{X}=$ $\left(X_{1}, \ldots, X_{|\omega|}\right) \in\left(\mathbb{K}^{s \times s}\right)^{d}, X_{|\omega|+1} \in \mathbb{K}^{s \times s}$ and $\underline{u} \in \mathbb{K}^{s}$, let

$$
\mathcal{T}\left(\left(\underline{\mathbf{A}}^{1}\right)^{\omega}(\underline{X}) \mathbf{B}_{k}^{1}\left(X_{|\omega|+1}\right) \underline{u}\right):=\left(\underline{\mathbf{A}}^{2}\right)^{\omega}(\underline{X}) \mathbf{B}_{k}^{2}\left(X_{|\omega|+1}\right) \underline{u}
$$

and extend it by linearity. We proceed by showing some properties of $\mathcal{T}$. 
- The domain of $\mathcal{T}$ is $\mathbb{K}^{L_{1}}$ : The domain of $\mathcal{T}$ consists of all the vectors in $\mathbb{K}^{L_{1}}$ which are in

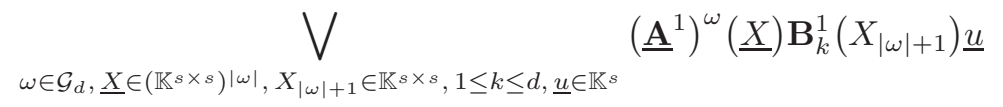

and that is exactly $\mathcal{C}_{\underline{\mathbf{A}}, \underline{\mathbf{B}}}=\mathbb{K}^{L_{1}}$, by the controllability of $\mathcal{R}_{1}$ and hence of $\left(\underline{\mathbf{A}}^{1}, \underline{\mathbf{B}}^{1}\right)$.

- $\underline{\mathcal{T}}$ is well-defined: Let $\underline{w}_{1}, \underline{w}_{2} \in \mathbb{K}^{L_{1}}$, then they can be written as

$$
\underline{w}_{1}=\sum_{j=1}^{p_{1}}\left(\underline{\mathbf{A}}^{1}\right)^{\omega_{1, j}}\left(X_{j, 1}^{(1)} \ldots, X_{j,\left|\omega_{1, j}\right|}^{(1)}\right) \mathbf{B}_{k_{1, j}}^{1}\left(X_{j,\left|\omega_{1, j}\right|+1}^{(1)}\right) \underline{u}_{1, j}
$$

and

$$
\underline{w}_{2}=\sum_{i=1}^{p_{2}}\left(\underline{\mathbf{A}}^{1}\right)^{\omega_{2, i}}\left(X_{i, 1}^{(2)}, \ldots, X_{i, \mid \omega_{2, i}}^{(2)}\right) \mathbf{B}_{k_{2, i}}^{1}\left(X_{i,\left|\omega_{2, i}\right|+1}^{(2)}\right) \underline{u}_{2, i}
$$

where $p_{1}, p_{2} \in \mathbb{N}, \omega_{1, j}, \omega_{2, i} \in \mathcal{G}_{d}, 1 \leq k_{1, j}, k_{2, i} \leq d, \underline{u}_{1, j}, \underline{u}_{2, i} \in \mathbb{K}^{s}$ and $X_{j, \alpha}^{(1)}, X_{i, \beta}^{(2)} \in$ $\mathbb{K}^{s \times s}$, for every $1 \leq \alpha \leq\left|\omega_{1, j}\right|, 1 \leq \beta \leq\left|\omega_{2, i}\right|, 1 \leq j \leq p_{1}$ and $1 \leq i \leq p_{2}$. Apply (2.14), so for every $\omega \in \mathcal{G}_{d}$,

$$
C^{1} \cdot\left(\underline{\mathbf{A}}^{1}\right)^{\omega} \cdot\left(\underline{\mathbf{A}}^{1}\right)^{\omega_{1, j}} \cdot \mathbf{B}_{k_{1, j}}^{1}=C^{2} \cdot\left(\underline{\mathbf{A}}^{2}\right)^{\omega} \cdot\left(\underline{\mathbf{A}}^{2}\right)^{\omega_{1, j}} \cdot \mathbf{B}_{k_{1, j}}^{2}
$$

and

$$
C^{1} \cdot\left(\underline{\mathbf{A}}^{1}\right)^{\omega} \cdot\left(\underline{\mathbf{A}}^{1}\right)^{\omega_{2, i}} \cdot \mathbf{B}_{k_{2, i}}^{1}=C^{2} \cdot\left(\underline{\mathbf{A}}^{2}\right)^{\omega} \cdot\left(\underline{\mathbf{A}}^{2}\right)^{\omega_{2, i}} \cdot \mathbf{B}_{k_{2, i}}^{2},
$$

which imply that for every $\underline{X} \in\left(\mathbb{K}^{s \times s}\right)^{|\omega|}$,

$$
\begin{gathered}
C^{2}\left(\underline{\mathbf{A}}^{2}\right)^{\omega}(\underline{X})\left[\sum_{j=1}^{p_{1}}\left(\underline{\mathbf{A}}^{2}\right)^{\omega_{1, j}}\left(X_{j, 1}^{(1)}, \ldots, X_{j,\left|\omega_{1, j}\right|}^{(1)}\right) \mathbf{B}_{k_{1, j}}^{2}\left(X_{j,\left|\omega_{1, j}\right|+1}^{(1)} \underline{u}_{1, j}\right]-C^{2}\left(\underline{\mathbf{A}}^{2}\right)^{\omega}(\underline{X})\right. \\
{\left[\sum_{i=1}^{p_{2}}\left(\underline{\mathbf{A}}^{2}\right)^{\omega_{2, i}}\left(X_{i, 1}^{(2)}, \ldots, X_{i,\left|\omega_{2, i}\right|}^{(2)}\right) \mathbf{B}_{k_{2, i}}^{2}\left(X_{i,\left|\omega_{2, i}\right|+1}^{(2)}\right) \underline{u}_{2, i}\right]=C^{1}\left(\underline{\mathbf{A}}^{1}\right)^{\omega}(\underline{X})\left[\sum_{j=1}^{p_{1}}\left(\underline{\mathbf{A}}^{1}\right)^{\omega_{1, j}}\right.} \\
\left(X_{j, 1}^{(1)}, \ldots, X_{j,\left|\omega_{1, j}\right|}^{(1)}\right) \mathbf{B}_{k_{1, j}}^{1}\left(X_{j,\left|\omega_{1, j}\right|+1}^{(1)} \underline{u}_{1, j}\right]-C^{1}\left(\underline{\mathbf{A}}^{1}\right)^{\omega}(\underline{X})\left[\sum _ { i = 1 } ^ { p _ { 2 } } ( \underline { \mathbf { A } } ^ { 1 } ) ^ { \omega _ { 2 , i } } \left(X_{i, 1}^{(2)}, \ldots,\right.\right. \\
\left.X_{\left.i, \mid \omega_{2, i}\right)}^{(2)}\right) \mathbf{B}_{k_{2, i}}^{1}\left(X_{i,\left|\omega_{2, i}\right|+1}^{(2)} \underline{u}_{2, i}\right],
\end{gathered}
$$

i.e.,

$$
C^{2}\left(\underline{\mathbf{A}}^{2}\right)^{\omega}(\underline{X})\left(\mathcal{T}\left(\underline{w}_{1}\right)-\mathcal{T}\left(\underline{w}_{2}\right)\right)=C^{1}\left(\underline{\mathbf{A}}^{1}\right)^{\omega}(\underline{X})\left(\underline{w}_{1}-\underline{w}_{2}\right) .
$$

Finally, if $\underline{w}_{1}=\underline{w}_{2}$, then $C^{2}\left(\underline{\mathbf{A}}^{2}\right)^{\omega}(\underline{X})\left(\mathcal{T}\left(\underline{w}_{1}\right)-\mathcal{T}\left(\underline{w}_{2}\right)\right)=\underline{0}$ for all $\omega \in \mathcal{G}_{d}$ and $\underline{X} \in\left(\mathbb{K}^{s \times s}\right)^{|\omega|}$, whereas the observability of $\mathcal{R}_{2}$ and hence of $\left(C^{2}, \underline{\mathbf{A}}^{2}\right)$ guarantees that $\mathcal{T}\left(\underline{w}_{1}\right)=\mathcal{T}\left(\underline{w}_{2}\right)$.

- $\mathcal{T}$ is $1-1$ : If $\underline{w}_{1}, \underline{w}_{2} \in \mathbb{K}^{L_{1}}$ such that $\mathcal{T}\left(\underline{w}_{1}\right)=\mathcal{T}\left(\underline{w}_{2}\right)$, it follows from (2.16) that $C^{1}\left(\underline{\mathbf{A}}^{1}\right)^{\omega}(\underline{X})\left(\underline{w}_{1}-\underline{w}_{2}\right)=\underline{0}$ for every $\omega \in \mathcal{G}_{d}$ and $\underline{X} \in\left(\mathbb{K}^{s \times s}\right)^{|\omega|}$, whereas the observability of $\mathcal{R}_{1}$ and hence of $\left(C^{1}, \underline{\mathbf{A}}^{1}\right)$ guarantees that $\underline{w}_{1}=\underline{w}_{2}$.

- $\underline{\mathcal{T}}$ is onto $\mathbb{K}^{L_{2}}$ : The tuple $\left(\underline{\mathbf{A}}^{2}, \underline{\mathbf{B}}^{2}\right)$ is controllable and hence every $\underline{e} \in \mathbb{K}^{L_{2}}$ can be written as

$$
\underline{e}=\sum_{j=1}^{p}\left(\underline{\mathbf{A}}^{2}\right)^{\nu_{j}}\left(\underline{X}^{(j)}\right) \mathbf{B}_{k_{j}}^{2}\left(X_{j+1}\right) \underline{v}_{j}=\mathcal{T}\left(\sum_{j=1}^{p}\left(\underline{\mathbf{A}}^{1}\right)^{\nu_{j}}\left(\underline{X}^{(j)}\right) \mathbf{B}_{k_{j}}^{1}\left(X_{j+1}\right) \underline{v}_{j}\right),
$$


M. PORAT AND V. VINNIKOV

where $p \in \mathbb{N}, \nu_{j} \in \mathcal{G}_{d}, \underline{X}^{(j)} \in\left(\mathbb{K}^{s \times s}\right)^{\left|\nu_{j}\right|}, X_{j+1} \in \mathbb{K}^{s \times s}, 1 \leq k_{j} \leq d$ and $\underline{v}_{j} \in \mathbb{K}^{s}$ for every $1 \leq j \leq p$, i.e., $\mathbb{K}^{L_{2}} \subseteq \operatorname{Im}(\mathcal{T})$ and hence $\mathcal{T}$ is onto $\mathbb{K}^{L_{2}}$.

Therefore, $\mathcal{T}: \mathbb{K}^{L_{1}} \rightarrow \mathbb{K}^{L_{2}}$ is an isomorphism, $L_{1}=L_{2}:=L$, the representative matrix $T:=[\mathcal{T}]_{\mathcal{E}_{L}} \in \mathbb{K}^{L \times L}$ is invertible and $\mathcal{T}(\underline{w})=T \underline{w}$ for all $\underline{w} \in \mathbb{K}^{L}$.

- The realizations $\mathcal{R}_{1}$ and $\mathcal{R}_{2}$ are similar: If $\underline{w}_{1}=\underline{0}$ and $\omega=\emptyset$, then (2.16) implies

$$
C^{2} T \underline{w}_{2}=C^{1} \underline{w}_{2}, \forall \underline{w}_{2} \in \mathbb{K}^{L} \Longrightarrow C^{1}=C^{2} T,
$$

while applying (2.16) again with $\underline{w}_{1}=0$ and using the observability of $\left(C^{2}, \underline{\mathbf{A}}^{2}\right)$, lead to

$$
\mathbf{A}_{k}^{1}(X)=T^{-1} \mathbf{A}_{k}^{2}(X) T, \forall X \in \mathbb{K}^{s \times s}, 1 \leq k \leq d .
$$

From the definition of $\mathcal{T}$ we have

$$
T \mathbf{B}_{k}^{1}(X) \underline{u}=\mathcal{T}\left(\mathbf{B}_{k}^{1}(X) \underline{u}\right)=\mathbf{B}_{k}^{2}(X) \underline{u}, \forall \underline{u} \in \mathbb{K}^{L} \Longrightarrow \mathbf{B}_{k}^{1}(X)=T^{-1} \mathbf{B}_{k}^{2}(X)
$$

for every $X \in \mathbb{K}^{s \times s}$ and $1 \leq k \leq d$. Thus, we proved the realizations are similar.

- $T$ is uniquely determined: Let $T_{2} \in \mathbb{K}^{L \times L}$ be such that $\mathbf{B}_{k}^{1}=T_{2}^{-1} \cdot \mathbf{B}_{k}^{2}$ and $\mathbf{A}_{k}^{1}=T_{2}^{-1} \cdot \mathbf{A}_{k}^{2} \cdot T_{2}$ for every $1 \leq k \leq d$, then it is easily seen that $T_{2}$ satisfies the relation in (2.15) and thus the controllability of $\left(\underline{\mathbf{A}}^{2}, \underline{\mathbf{B}}^{2}\right)$ implies that $T_{2}=T$.

- Moreover,

$$
\begin{array}{r}
I_{L m}-\sum_{k=1}^{d}\left(X_{k}-I_{m} \otimes Y_{k}\right) \mathbf{A}_{k}^{2}=I_{L m}-\sum_{k=1}^{d}\left[\left(I_{m} \otimes T\right)\left(X_{k}-I_{m} \otimes Y_{k}\right) \mathbf{A}_{k}^{1}\left(I_{m} \otimes T^{-1}\right)\right] \\
=\left(I_{m} \otimes T\right)\left(I_{L m}-\sum_{k=1}^{d}\left(X_{k}-I_{m} \otimes Y_{k}\right) \mathbf{A}_{k}^{1}\right)\left(I_{m} \otimes T\right)^{-1}
\end{array}
$$

for every $m \in \mathbb{N}$, which implies that $\underline{X} \in D O M_{s m}\left(\mathcal{R}_{2}\right) \Longleftrightarrow \underline{X} \in D O M_{s m}\left(\mathcal{R}_{1}\right)$, i.e., that $D O M_{s m}\left(\mathcal{R}_{1}\right)=D O M_{s m}\left(\mathcal{R}_{2}\right)$. It is easily seen that using the relations in (2.13), we have $\mathcal{R}_{1}(\underline{X})=\mathcal{R}_{2}(\underline{X})$ for every $\underline{X} \in D O M_{s m}\left(\mathcal{R}_{1}\right)$.

- Finally, the relations in $(2.13)$ also imply that $C^{2} \otimes 1_{\mathcal{A}}=\left(C^{1} \otimes 1_{\mathcal{A}}\right)\left(T^{-1} \otimes 1_{\mathcal{A}}\right)$ and that for every $\underline{\mathfrak{A}}=\left(\mathfrak{A}_{1}, \ldots, \mathfrak{A}_{d}\right) \in\left(\mathcal{A}^{s \times s}\right)^{d}$, we have

$\left(\mathfrak{A}_{k}\right)\left(\mathbf{B}_{k}^{2}\right)^{\mathcal{A}}=\left(T \otimes 1_{\mathcal{A}}\right)\left(\mathfrak{A}_{k}\right)\left(\mathbf{B}_{k}^{1}\right)^{\mathcal{A}}$ and $\left(\mathfrak{A}_{k}\right)\left(\mathbf{A}_{k}^{2}\right)^{\mathcal{A}}=\left(T \otimes 1_{\mathcal{A}}\right)\left(\mathfrak{A}_{k}\right)\left(\mathbf{A}_{k}^{1}\right)^{\mathcal{A}}\left(T^{-1} \otimes 1_{\mathcal{A}}\right)$

for every $1 \leq k \leq d$. Therefore,

$$
\begin{gathered}
I_{L} \otimes 1_{\mathcal{A}}-\sum_{k=1}^{d}\left(\mathfrak{A}_{k}-Y_{k} \otimes 1_{\mathcal{A}}\right)\left(\mathbf{A}_{k}^{2}\right)^{\mathcal{A}}=I_{L} \otimes 1_{\mathcal{A}}-\sum_{k=1}^{d}\left[\left(T \otimes 1_{\mathcal{A}}\right)\left(\mathfrak{A}_{k}-Y_{k} \otimes 1_{\mathcal{A}}\right)\left(\mathbf{A}_{k}^{1}\right)^{\mathcal{A}}\right. \\
\left.\left(T^{-1} \otimes 1_{\mathcal{A}}\right)\right]=\left(T \otimes 1_{\mathcal{A}}\right)\left(I_{L} \otimes 1_{\mathcal{A}}-\sum_{k=1}^{d}\left(\mathfrak{A}_{k}-Y_{k} \otimes 1_{\mathcal{A}}\right)\left(\mathbf{A}_{k}^{1}\right)^{\mathcal{A}}\right)\left(T \otimes 1_{\mathcal{A}}\right)^{-1}
\end{gathered}
$$

which implies that $D O M^{\mathcal{A}}\left(\mathcal{R}_{1}\right)=D O M^{\mathcal{A}}\left(\mathcal{R}_{2}\right)$ and that $\mathcal{R}_{1}^{\mathcal{A}}(\underline{\mathfrak{A}})=\mathcal{R}_{2}^{\mathcal{A}}(\underline{\mathfrak{A}})$ for every $\underline{\mathfrak{A}} \in D O M^{\mathcal{A}}\left(\mathcal{R}_{1}\right)$.

Remark 2.14. One can obtain the equality in (2.14) and hence prove Theorem 2.13 without using Lemma 2.12, by evaluating nc rational expressions on generic matrices and then considering power series in commuting variables (the entries of the generic matrices). 
2.4. Kalman decomposition. We proceed next to obtain a Kalman decomposition for nc Fornasini-Marchesini realizations centred at a matrix point $\underline{Y} \in\left(\mathbb{K}^{s \times s}\right)^{d}$, which generalizes the Kalman decomposition for nc Fornasini-Marchesini realizations centred at a scalar point (as in [8]), where the later decomposition is a generalization of the classical Kalman decomposition (see [13, 50]).

This is the first place in our analysis where $\mathcal{A}$ is no longer an arbitrary unital $\mathbb{K}$-algebra, but has to be stably finite. The stably finiteness is used to deduce the invertibility of one of the blocks in a block upper triangular matrix that is invertible (cf. Lemma 1.3).

Theorem 2.15 (Kalman Decomposition). Let

$$
\mathcal{R}(\underline{X})=D+C\left(I_{L}-\sum_{k=1}^{d} \mathbf{A}_{k}\left(X_{k}-Y_{k}\right)\right)^{-1} \sum_{k=1}^{d} \mathbf{B}_{k}\left(X_{k}-Y_{k}\right)
$$

be a nc Fornasini-Marchesini realization centred at $\underline{Y} \in\left(\mathbb{K}^{s \times s}\right)^{d}$. There exists a nc Fornasini-Marchesini realization $\widetilde{\mathcal{R}}$ centred at $\underline{Y}$, that is controllable and observable, of dimension $\widetilde{L}=\operatorname{dim}\left(\mathcal{C}_{\underline{\mathbf{A}}, \underline{\mathbf{B}}}\right)-\operatorname{dim}\left(\mathcal{C}_{\underline{\mathbf{A}}, \underline{\mathbf{B}}} \cap \mathcal{N} \mathcal{O}_{C, \underline{\mathbf{A}}}\right)$, such that

$$
D O M_{s m}(\mathcal{R}) \subseteq D O M_{s m}(\widetilde{\mathcal{R}}) \text { and } \mathcal{R}(\underline{X})=\widetilde{\mathcal{R}}(\underline{X}), \forall \underline{X} \in D O M_{s m}(\mathcal{R})
$$

for every $m \in \mathbb{N}$, and for any unital stably finite $\mathbb{K}$-algebra $\mathcal{A}$,

$$
D O M^{\mathcal{A}}(\mathcal{R}) \subseteq D O M^{\mathcal{A}}(\widetilde{\mathcal{R}}) \text { and } \mathcal{R}^{\mathcal{A}}(\underline{\mathfrak{A}})=\widetilde{\mathcal{R}}^{\mathcal{A}}(\underline{\mathfrak{A}}), \forall \underline{\mathfrak{A}} \in D O M^{\mathcal{A}}(\mathcal{R}) .
$$

As the proof shows (see (2.19) below), $\widetilde{\mathcal{R}}$ is obtained from $\mathcal{R}$ analogously to the classical case, by restricting to a joint invariant subspace of the operators $\mathbf{A}_{1}, \ldots, \mathbf{A}_{d}$ and then compressing to a co-invariant subspace.

Proof. Using the controllability and un-observability subspaces of $\mathbb{K}^{L}$, which correspond to $(\underline{\mathbf{A}}, \underline{\mathbf{B}})$ and $(C, \underline{\mathbf{A}})$, define $\mathcal{C}:=\mathcal{C}_{\underline{\mathbf{A}}, \underline{\mathbf{B}}}, \mathcal{N O}:=\mathcal{N} \mathcal{O}_{C, \underline{\mathbf{A}}}$,

$$
\mathcal{H}_{1}:=\mathcal{N O} \cap \mathcal{C},
$$

$\mathcal{H}_{2}$ a complementary subspace of $\mathcal{H}_{1}$ in $\mathcal{C}$ and $\mathcal{H}_{3}$ a complementary subspace of $\mathcal{H}_{1}$ in $\mathcal{N O}$, thus

$$
\mathcal{C}=\mathcal{H}_{2}+\mathcal{H}_{1} \text { and } \mathcal{N O}=\mathcal{H}_{1}+\mathcal{H}_{3} \text {. }
$$

If $h_{1}+h_{2}+h_{3}=0$ where $h_{1} \in \mathcal{H}_{1}, h_{2} \in \mathcal{H}_{2}$ and $h_{3} \in \mathcal{H}_{3}$, then $h_{1}+h_{2}=-h_{3} \in$ $\mathcal{C} \cap \mathcal{N O} \cap \mathcal{H}_{3}=\mathcal{H}_{1} \cap \mathcal{H}_{3}=\{\underline{0}\}$, which implies that $h_{1}+h_{2}=-h_{3}=\underline{0}$ and hence $h_{1}=h_{2}=h_{3}=\underline{0}$. Therefore, the sum $\mathcal{H}_{1}+\mathcal{H}_{2}+\mathcal{H}_{3}$ is a direct sum; let $\mathcal{H}_{4}$ be a complementary subspace of $\mathcal{H}_{1}+\mathcal{H}_{2}+\mathcal{H}_{3}$ in $\mathbb{K}^{L}$, thus

$$
\mathbb{K}^{L}=\mathcal{H}_{2}+\mathcal{H}_{1} \dot{+} \mathcal{H}_{4} \dot{+} \mathcal{H}_{3} \text {. }
$$

Notice that we are interested in $\mathcal{H}_{2}$ as it is a subspace of a controllable (invariant) subspace and a complementary subspace of an un-observable subspace. Define the dimensions $L_{j}:=\operatorname{dim}\left(\mathcal{H}_{j}\right)$ for $1 \leq j \leq 4$, so $L=L_{1}+\ldots+L_{4}$. From Proposition 2.8 we have $\mathcal{N} \mathcal{O}^{(m)}=\mathcal{N} \mathcal{O} \otimes \mathbb{K}^{m}$ and $\mathcal{C}^{(m)}=\mathcal{C} \otimes \mathbb{K}^{m}$ for every $m \in \mathbb{N}$, hence

$$
\mathcal{H}_{1}^{(m)}:=\mathcal{N O}^{(m)} \cap \mathcal{C}^{(m)}=\left(\mathcal{N O} \otimes \mathbb{K}^{m}\right) \cap\left(\mathcal{C} \otimes \mathbb{K}^{m}\right)=\mathcal{H}_{1} \otimes \mathbb{K}^{m} .
$$

Define $\mathcal{H}_{2}^{(m)}:=\mathcal{H}_{2} \otimes \mathbb{K}^{m}, \mathcal{H}_{3}^{(m)}:=\mathcal{H}_{3} \otimes \mathbb{K}^{m}$ and $\mathcal{H}_{4}^{(m)}:=\mathcal{H}_{4} \otimes \mathbb{K}^{m}$, thus

$$
\mathcal{H}_{2}^{(m)} \dot{+} \mathcal{H}_{1}^{(m)}=\mathcal{C}^{(m)}, \mathcal{H}_{1}^{(m)} \dot{+} \mathcal{H}_{3}^{(m)}=\mathcal{N O}^{(m)}
$$


and

$$
\mathbb{K}^{L m}=\mathcal{H}_{2}^{(m)} \dot{+} \mathcal{H}_{1}^{(m)} \dot{+} \mathcal{H}_{4}^{(m)} \dot{+} \mathcal{H}_{3}^{(m)} .
$$

- With respect to the decomposition (2.19) of $\mathbb{K}^{L}$, there exists $P \in \mathbb{K}^{L \times L}$ invertible such that for every $X \in \mathbb{K}^{s m \times s m}$ and $1 \leq k \leq d$, the matrices $(X) \mathbf{A}_{k} \in$ $\mathbb{K}^{L m \times L m},(X) \mathbf{B}_{k} \in \mathbb{K}^{L m \times s m}$ and $I_{m} \otimes C \in \mathbb{K}^{s m \times L m}$ can be decomposed, w.r.t $(2.20)$, as

$$
\begin{aligned}
&\left(P^{(m)}\right)^{-1}(X) \mathbf{A}_{k} P^{(m)}=(X) {\left[\begin{array}{cccc}
\mathbf{A}_{k}^{1,1} & \mathbf{A}_{k}^{1,2} & \mathbf{A}_{k}^{1,3} & \mathbf{A}_{k, 4}^{1,4} \\
\mathbf{A}_{k}^{2,1} & \mathbf{A}_{k}^{2,2} & \mathbf{A}_{k}^{2,3} & \mathbf{A}_{k}^{2,4} \\
\mathbf{A}_{k}^{3,1} & \mathbf{A}_{k}^{3,2} & \mathbf{A}_{k}^{3,3} & \mathbf{A}_{k}^{3,4} \\
\mathbf{A}_{k}^{4,1} & \mathbf{A}_{k}^{4,2} & \mathbf{A}_{k}^{4,3} & \mathbf{A}_{k}^{4,4}
\end{array}\right],\left(P^{(m)}\right)^{-1}(X) \mathbf{B}_{k}=(X)\left[\begin{array}{c}
\mathbf{B}_{k}^{1} \\
\mathbf{B}_{k}^{2} \\
\mathbf{B}_{k}^{3} \\
\mathbf{B}_{k}^{4}
\end{array}\right] } \\
& \text { and }\left(I_{m} \otimes C\right) P^{(m)}=\left[\begin{array}{llll}
C^{1} & C^{2} & C^{3} & C^{4}
\end{array}\right],
\end{aligned}
$$

where $P^{(m)}:=I_{m} \otimes P$. If $\underline{u}_{1} \in \mathcal{H}_{1}^{(m)}, \underline{u}_{2} \in \mathcal{H}_{2}^{(m)}, \underline{u}_{3} \in \mathcal{H}_{3}^{(m)}$ and $\underline{v} \in \mathbb{K}^{s m}$, then

$$
\begin{array}{r}
(X) \mathbf{A}_{k} \underline{u}_{1} \in \mathcal{N} \mathcal{O}^{(m)} \cap \mathcal{C}^{(m)}=\mathcal{H}_{1}^{(m)},(X) \mathbf{A}_{k} \underline{u}_{2} \in \mathcal{C}^{(m)},(X) \mathbf{A}_{k} \underline{u}_{3} \in \mathcal{N} \mathcal{O}^{(m)}, \\
(X) \mathbf{B}_{k} \underline{v} \in \mathcal{C}^{(m)} \text { and }\left(I_{m} \otimes C\right) \underline{u}_{1}=\underline{0},
\end{array}
$$

which imply that

$$
(X) \mathbf{A}_{k}^{1,2},(X) \mathbf{A}_{k}^{3,2},(X) \mathbf{A}_{k}^{4,2},(X) \mathbf{A}_{k}^{3,1},(X) \mathbf{A}_{k}^{4,1},(X) \mathbf{A}_{k}^{1,4},(X) \mathbf{A}_{k}^{3,4},(X) \mathbf{B}_{k}^{3},(X) \mathbf{B}_{k}^{4}, C^{2}, C^{4}
$$

all vanish. Therefore, we get

$$
\begin{gathered}
\left(P^{(m)}\right)^{-1}(X) \mathbf{A}_{k} P^{(m)}=(X)\left[\begin{array}{cccc}
\mathbf{A}_{k}^{1,1} & 0 & \mathbf{A}_{k}^{1,3} & 0 \\
\mathbf{A}_{k}^{2,1} & \mathbf{A}_{k}^{2,2} & \mathbf{A}_{k}^{2,3} & \mathbf{A}_{k}^{2,4} \\
0 & 0 & \mathbf{A}_{k}^{3,3} & 0 \\
0 & 0 & \mathbf{A}_{k}^{4,3} & \mathbf{A}_{k}^{4,4}
\end{array}\right],\left(P^{(m)}\right)^{-1}(X) \mathbf{B}_{k}=(X)\left[\begin{array}{c}
\mathbf{B}_{k}^{1} \\
\mathbf{B}_{k}^{2} \\
0 \\
0
\end{array}\right] \\
\text { and }\left(I_{m} \otimes C\right) P^{(m)}=\left[\begin{array}{llll}
C^{1} & 0 & C^{3} & 0
\end{array}\right],
\end{gathered}
$$

hence for every $\underline{X} \in D O M_{s m}(\mathcal{R})$,

$$
\begin{gathered}
\mathcal{R}(\underline{X})=I_{m} \otimes D+\left(I_{m} \otimes C\right) P^{(m)}\left(I_{L m}-\sum_{k=1}^{d}\left[\left(P^{(m)}\right)^{-1}\left(X_{k}-I_{m} \otimes Y_{k}\right) \mathbf{A}_{k} P^{(m)}\right]\right)^{-1} \\
\sum_{k=1}^{d}\left[\left(P^{(m)}\right)^{-1}\left(X_{k}-I_{m} \otimes Y_{k}\right) \mathbf{B}_{k}\right]=I_{m} \otimes D+\left[\begin{array}{llll}
C^{1} & 0 & C^{3} & 0
\end{array}\right]\left[\begin{array}{cccc}
\lambda_{1,1} & 0 & \lambda_{1,3} & 0 \\
\lambda_{2,1} & \lambda_{2,2} & \lambda_{2,3} & \lambda_{2,4} \\
0 & 0 & \lambda_{3,3} & 0 \\
0 & 0 & \lambda_{4,3} & \lambda_{4,4}
\end{array}\right]^{-1} \\
\sum_{k=1}^{d}\left(X_{k}-I_{m} \otimes Y_{k}\right)\left[\begin{array}{c}
\mathbf{B}_{k}^{1} \\
\mathbf{B}_{k}^{2} \\
0 \\
0
\end{array}\right],
\end{gathered}
$$

where

$$
\Lambda_{X}:=\left[\begin{array}{cccc}
\lambda_{11} & 0 & \lambda_{13} & 0 \\
\lambda_{21} & \lambda_{22} & \lambda_{23} & \lambda_{24} \\
0 & 0 & \lambda_{33} & 0 \\
0 & 0 & \lambda_{43} & \lambda_{44}
\end{array}\right]=I_{L m}-\sum_{k=1}^{d}\left(X_{k}-I_{m} \otimes Y_{k}\right)\left[\begin{array}{cccc}
\mathbf{A}_{k}^{1,1} & 0 & \mathbf{A}_{k}^{1,3} & 0 \\
\mathbf{A}_{k}^{2,1} & \mathbf{A}_{k}^{2,2} & \mathbf{A}_{k}^{2,3} & \mathbf{A}_{k}^{2,4} \\
0 & 0 & \mathbf{A}_{k}^{3,3} & 0 \\
0 & 0 & \mathbf{A}_{k}^{4,3} & \mathbf{A}_{k}^{4,4}
\end{array}\right]
$$


is invertible. Thus

$$
\operatorname{det}\left(\lambda_{11}\right)=\operatorname{det}\left(I_{\widetilde{L} m}-\sum_{k=1}^{d}\left(X_{k}-I_{m} \otimes Y_{k}\right) \mathbf{A}_{k}^{1,1}\right) \neq 0
$$

where $\widetilde{L}=L_{2}=\operatorname{dim}(\mathcal{C})-\operatorname{dim}(\mathcal{N O} \cap \mathcal{C})$ and the inverse of $\Lambda_{X}$ is given by

$$
\Lambda_{X}^{-1}=\left[\begin{array}{cccc}
\lambda_{11}^{-1} & 0 & -\lambda_{11}^{-1} \lambda_{13} \lambda_{33}^{-1} & 0 \\
-\lambda_{22}^{-1} \lambda_{21} \lambda_{11}^{-1} & \lambda_{22}^{-1} & \lambda_{22}^{-1}\left(\lambda_{24} \lambda_{44}^{-1} \lambda_{43}-\lambda_{23}+\lambda_{21} \lambda_{11}^{-1} \lambda_{13}\right) \lambda_{33}^{-1} & -\lambda_{22}^{-1} \lambda_{24} \lambda_{44}^{-1} \\
0 & 0 & \lambda_{33}^{-1} & 0 \\
0 & 0 & -\lambda_{44}^{-1} \lambda_{43} \lambda_{33}^{-1} & \lambda_{44}^{-1}
\end{array}\right] .
$$

Therefore, for every $\underline{X} \in D O M_{s m}(\mathcal{R})$, we have

$$
\mathcal{R}(\underline{X})=\widetilde{\mathcal{R}}(\underline{X}):=I_{m} \otimes D+\left(I_{m} \otimes C^{1}\right)\left(I_{m \tilde{L}^{-}} \sum_{k=1}^{d}\left(X_{k}-I_{m} \otimes Y_{k}\right) \mathbf{A}_{k}^{1,1}\right)^{-1} \sum_{k=1}^{d}\left(X_{k}-I_{m} \otimes Y_{k}\right) \mathbf{B}_{k}^{1}
$$

where $\widetilde{\mathcal{R}}$ is the nc Fornasini-Marchesini realization described by $\left(\widetilde{L}, D, C^{1}, \underline{\mathbf{A}}^{1,1}, \underline{\mathbf{B}}^{1}\right)$ and centred at $\underline{Y}$, and $(2.21)$ implies that $\underline{X} \in D O M_{s m}(\widetilde{\mathcal{R}})$.

- Next, let $\underline{\mathfrak{A}}=\left(\mathfrak{A}_{1}, \ldots, \mathfrak{A}_{d}\right) \in\left(\mathcal{A}^{s \times s}\right)^{d}$ and write $\mathfrak{A}_{k}=\sum_{i, j=1}^{s} E_{i j} \otimes \mathfrak{a}_{i j}^{(k)}$, where $E_{i j} \in \mathcal{E}_{s}$ and $\mathfrak{a}_{i j}^{(k)} \in \mathcal{A}$, then

$$
\begin{aligned}
& I_{L} \otimes 1_{\mathcal{A}}-\sum_{k=1}^{d}\left(\mathfrak{A}_{k}-Y_{k} \otimes 1_{\mathcal{A}}\right) \mathbf{A}_{k}^{\mathcal{A}}=I_{L} \otimes 1_{\mathcal{A}}-\sum_{k=1}^{d}\left[\sum_{i, j=1}^{s} \mathbf{A}_{k}\left(E_{i j}\right) \otimes \mathfrak{a}_{i j}^{(k)}-\mathbf{A}_{k}\left(Y_{k}\right) \otimes 1_{\mathcal{A}}\right] \\
& =I_{L} \otimes 1_{\mathcal{A}}-\sum_{k=1}^{d} \sum_{i, j=1}^{s}\left[P\left[\begin{array}{cccc}
\mathbf{A}_{k, 1}^{1,1} & 0 & \mathbf{A}_{k, 3}^{1,3} & 0 \\
\mathbf{A}_{k}^{2,1} & \mathbf{A}_{k}^{2,2} & \mathbf{A}_{k}^{2,3} & \mathbf{A}_{k}^{2,4} \\
0 & 0 & \mathbf{A}_{k}^{3,3} & 0 \\
0 & 0 & \mathbf{A}_{k}^{4,3} & \mathbf{A}_{k}^{4,4}
\end{array}\right]\left(E_{i j}\right) P^{-1}\right] \otimes \mathfrak{a}_{i j}^{(k)}-\sum_{k=1}^{d}[P \\
& \left.\left[\begin{array}{cccc}
\mathbf{A}_{k}^{1,1} & 0 & \mathbf{A}_{k}^{1,3} & 0 \\
\mathbf{A}_{k}^{2,1} & \mathbf{A}_{k}^{2,2} & \mathbf{A}_{k}^{2,3} & \mathbf{A}_{k}^{2,4} \\
0 & 0 & \mathbf{A}_{k}^{3,3} & 0 \\
0 & 0 & \mathbf{A}_{k}^{4,3} & \mathbf{A}_{k}^{4,4}
\end{array}\right]\left(Y_{k}\right) P^{-1}\right] \otimes 1_{\mathcal{A}}=\left(P \otimes 1_{\mathcal{A}}\right)\left[\begin{array}{cccc}
\lambda_{1, \mathcal{A}} & 0 & * & 0 \\
* & * & * & * \\
0 & 0 & * & 0 \\
0 & 0 & * & *
\end{array}\right]\left(P \otimes 1_{\mathcal{A}}\right)^{-1},
\end{aligned}
$$

where

$$
\lambda_{1, \mathcal{A}}:=I_{\widetilde{L}} \otimes 1_{\mathcal{A}}-\sum_{k=1}^{d}\left[\sum_{i, j=1}^{s} \mathbf{A}_{k}^{1,1}\left(E_{i j}\right) \otimes \mathfrak{a}_{i j}^{(k)}-\mathbf{A}_{k}^{1,1}\left(Y_{k}\right) \otimes 1_{\mathcal{A}}\right] .
$$

Therefore, if $\underline{\mathfrak{A}} \in D O M^{\mathcal{A}}(\mathcal{R})$, then

$$
\left(P \otimes 1_{\mathcal{A}}\right)^{-1}\left(I_{L} \otimes 1_{\mathcal{A}}-\sum_{k=1}^{d}\left(\mathfrak{A}_{k}-Y_{k} \otimes 1_{\mathcal{A}}\right) \mathbf{A}_{k}^{\mathcal{A}}\right)\left(P \otimes 1_{\mathcal{A}}\right)=\left[\begin{array}{cccc}
\lambda_{1, \mathcal{A}} & 0 & * & 0 \\
* & * & * & * \\
0 & 0 & * & 0 \\
0 & 0 & * & *
\end{array}\right]
$$

is invertible in $\mathcal{A}^{L \times L}$, while applying Lemma 1.3 to obtain that

$$
\lambda_{1, \mathcal{A}}=I_{\widetilde{L}} \otimes 1_{\mathcal{A}}-\sum_{k=1}^{d}\left(\mathfrak{A}_{k}-Y_{k} \otimes 1_{\mathcal{A}}\right)\left(\mathbf{A}_{k}^{1,1}\right)^{\mathcal{A}}
$$


is invertible in $\mathcal{A}^{\widetilde{L} \times \widetilde{L}}$, i.e., that $\underline{\mathfrak{A}} \in D O M^{\mathcal{A}}(\widetilde{\mathcal{R}})$. Moreover, a careful similar computation shows that if $\underline{\mathfrak{A}} \in D O M^{\mathcal{A}}(\mathcal{R})$, then $\mathcal{R}^{\mathcal{A}}(\underline{\mathfrak{A}})=\widetilde{\mathcal{R}}^{\mathcal{A}}(\underline{\mathfrak{A}})$.

- For every $\underline{u} \in \mathbb{K}^{s}, 1 \leq k \leq d$, a word $\omega=g_{i_{1}} \ldots g_{i_{\ell}} \in \mathcal{G}_{d}$ of length $\ell \in \mathbb{N}$ and $X_{1}, \ldots, X_{\ell+1} \in \mathbb{K}^{s \times s}$, we have

$$
\begin{aligned}
\underline{\mathbf{A}}^{\omega}\left(X_{1}, \ldots, X_{\ell}\right) \mathbf{B}_{k}\left(X_{\ell+1}\right) \underline{u} & =P\left(P^{-1} \mathbf{A}_{i_{1}}(X) P\right) \cdots\left(P^{-1} \mathbf{A}_{i_{\ell}}\left(X_{\ell}\right) P\right) P^{-1} \mathbf{B}_{k}\left(X_{\ell+1}\right) \underline{u} \\
& =P\left[\begin{array}{c}
\left(\underline{\mathbf{A}}^{1,1}\right)^{\omega}\left(X_{1}, \ldots, X_{\ell}\right) \mathbf{B}_{k}^{1}\left(X_{\ell+1}\right) \underline{u} \\
* \\
0 \\
0
\end{array}\right] .
\end{aligned}
$$

Thus, for every $\underline{v} \in \mathcal{C}$, we have $P^{-1} \underline{v} \in \widetilde{\mathcal{C}} \oplus \mathbb{K}^{L_{1}} \oplus\{\underline{0}\} \oplus\{\underline{0}\} \subseteq \mathbb{K}^{L}$ and hence $\operatorname{dim}(\mathcal{C}) \leq \operatorname{dim}(\widetilde{\mathcal{C}})+L_{1}$. As $\operatorname{dim}(\mathcal{C})=L_{1}+L_{2}$, we get that $L_{2} \leq \operatorname{dim}(\widetilde{\mathcal{C}})$, while $\widetilde{\mathcal{C}} \subseteq \mathbb{K}^{L_{2}}$ yields that $\widetilde{\mathcal{C}}=\mathbb{K}^{L_{2}}=\mathbb{K}^{\widetilde{L}}$, i.e., the realization $\widetilde{\mathcal{R}}$ is controllable.

- For every $\underline{w}_{1} \in \mathbb{K}^{L_{1}}, \underline{w}_{2} \in \mathbb{K}^{L_{2}}, \underline{w}_{3} \in \mathbb{K}^{L_{3}}$, a word $\omega=g_{i_{1}} \ldots g_{i_{\ell}} \in \mathcal{G}_{d}$ of length $\ell \in \mathbb{N}$ and $X_{1}, \ldots, X_{\ell} \in \mathbb{K}^{s \times s}$, we have

$$
\begin{aligned}
& C \underline{\mathbf{A}}^{\omega}\left(X_{1}, \ldots, X_{\ell}\right) P\left[\underline{w}_{2}^{T} \quad \underline{w}_{1}^{T} \quad 0 \quad \underline{w}_{3}^{T}\right]^{T}=C P\left(P^{-1} \mathbf{A}_{i_{1}}\left(X_{1}\right) P\right) \cdots\left(P^{-1} \mathbf{A}_{i_{\ell}}\left(X_{\ell}\right) P\right) \\
& {\left[\begin{array}{llll}
\underline{w}_{2}^{T} & \underline{w}_{1}^{T} & 0 & \underline{w}_{3}^{T}
\end{array}\right]^{T}=C P\left[\begin{array}{c}
\mathbf{A}_{i_{1}}^{1,1}\left(X_{1}\right) \cdots \mathbf{A}_{i_{\ell}}^{1,1}\left(X_{\ell}\right) \underline{w}_{2} \\
* \\
0 \\
*
\end{array}\right]=C^{1}\left(\underline{\mathbf{A}}^{1,1}\right)^{\omega}\left(X_{1}, \ldots, X_{\ell}\right) \underline{w}_{2},}
\end{aligned}
$$

therefore $\underline{w}_{2} \in \widetilde{\mathcal{N O}}:=\mathcal{N} \mathcal{O}_{C^{1}, \underline{\mathbf{A}}^{1,1}}$ implies that $P\left[\underline{w}_{2}^{T} \quad \underline{w}_{1}^{T} \quad 0 \quad \underline{w}_{3}^{T}\right]^{T} \in \mathcal{N O}$. As $P$ is invertible, $\operatorname{dim}(\mathcal{N O}) \geq \operatorname{dim}(\widetilde{\mathcal{N O}})+L_{1}+L_{3}=\operatorname{dim}(\widetilde{\mathcal{N O}})+\operatorname{dim}(\mathcal{N O})$ which guarantees that $\widetilde{\mathcal{N O}}=\{\underline{0}\}$, i.e., that $\widetilde{\mathcal{R}}$ is observable.

As a corollary we get that a nc Fornasini-Marchesini realization (of a nc rational expression) is minimal if and only if it is both controllable and observable:

Theorem 2.16. Let $R$ be a nc rational expression in $x_{1}, \ldots, x_{d}$ over $\mathbb{K}$ and $\mathcal{R}$ be a nc Fornasini-Marchesini realization of $R$ centred at $\underline{Y} \in\left(\mathbb{K}^{s \times s}\right)^{d}$. Then $\mathcal{R}$ is minimal if and only if $\mathcal{R}$ is controllable and observable.

Proof. If $\mathcal{R}$ is minimal, then using Theorem 2.15 we must have

$$
L \leq \widetilde{L}=\operatorname{dim}\left(\mathcal{C}^{(1)}\right)-\operatorname{dim}\left(\mathcal{C}^{(1)} \cap \mathcal{N O}^{(1)}\right) \leq L,
$$

which implies that $\widetilde{L}=L$, $\operatorname{dim}\left(\mathcal{C}^{(1)}\right)=L$ and $\operatorname{dim}\left(\mathcal{N O}^{(1)}\right)=0$, i.e., that $\mathcal{R}$ is controllable and observable.

On the other hand, let $\mathcal{R}$ be both controllable and observable, and suppose $\mathcal{R}^{\prime}$ is a minimal nc Fornasini-Marchesini realization of $R$ centred at $\underline{Y}$ of dimension $L^{\prime}$. Thus, by the first part of the theorem, $\mathcal{R}^{\prime}$ is controllable and observable, as well as $\mathcal{R}$, so Theorem 2.13 implies that $L=L^{\prime}$ and hence $\mathcal{R}$ is minimal.

Minimal nc Fornasini-Marchesini realizations are playing a central role in the analysis of the domains; one of the reasons is that they admit the maximal domain among all nc Fornasini-Marchesini realizations - of a given nc rational expressionwhich are centred at the same point: 
Lemma 2.17. Let $\mathcal{R}_{1}, \mathcal{R}_{2}$ be two nc Fornasini-Marchesini realizations of a nc rational expression $R$, both centred at $\underline{Y} \in\left(\mathbb{K}^{s \times s}\right)^{d}$. If $\mathcal{R}_{2}$ is minimal, then

$$
D O M_{s m}\left(\mathcal{R}_{1}\right) \subseteq D O M_{s m}\left(\mathcal{R}_{2}\right) \text { and } \mathcal{R}_{1}(\underline{X})=\mathcal{R}_{2}(\underline{X}), \forall \underline{X} \in D O M_{s m}\left(\mathcal{R}_{1}\right)
$$

for every $m \in \mathbb{N}$, and for any unital stably finite $\mathbb{K}$-algebra $\mathcal{A}$ :

$$
D O M^{\mathcal{A}}\left(\mathcal{R}_{1}\right) \subseteq D O M^{\mathcal{A}}\left(\mathcal{R}_{2}\right) \text { and } \mathcal{R}_{1}^{\mathcal{A}}(\underline{\mathfrak{A}})=\mathcal{R}_{2}^{\mathcal{A}}(\underline{\mathfrak{A}}), \forall \underline{\mathfrak{A}} \in D O M^{\mathcal{A}}\left(\mathcal{R}_{1}\right)
$$

Proof. Applying Theorem 2.15 for the nc Fornasini-Marchesini realization $\mathcal{R}_{1}$, there exists a minimal nc Fornasini-Marchesini realization $\widetilde{\mathcal{R}_{1}}$ centred at $\underline{Y}$ for which

$$
D O M_{s m}\left(\mathcal{R}_{1}\right) \subseteq D O M_{s m}\left(\widetilde{\mathcal{R}_{1}}\right) \text { and } \mathcal{R}_{1}(\underline{X})=\widetilde{\mathcal{R}_{1}}(\underline{X}), \forall \underline{X} \in D O M_{s m}\left(\mathcal{R}_{1}\right)
$$

for every $m \in \mathbb{N}$, and for any unital stably finite $\mathbb{K}$-algebra $\mathcal{A}$ :

$$
D O M^{\mathcal{A}}\left(\mathcal{R}_{1}\right) \subseteq D O M^{\mathcal{A}}\left(\widetilde{\mathcal{R}_{1}}\right) \text { and } \mathcal{R}_{1}^{\mathcal{A}}(\underline{\mathfrak{A}})=\widetilde{\mathcal{R}}^{\mathcal{A}}(\underline{\mathfrak{A}}), \forall \underline{\mathfrak{A}} \in D O M^{\mathcal{A}}\left(\mathcal{R}_{1}\right) .
$$

In particular $\widetilde{\mathcal{R}_{1}}$ is a nc Fornasini-Marchesini realization of $R$. As both $\mathcal{R}_{2}$ and $\widetilde{\mathcal{R}_{1}}$ are minimal nc Fornasini-Marchesini realizations of $R$, both centred at $\underline{Y}$, Theorem 2.13 implies that

$$
D O M_{s m}\left(\widetilde{\mathcal{R}_{1}}\right)=D O M_{s m}\left(\mathcal{R}_{2}\right) \text { and } \widetilde{\mathcal{R}_{1}}(\underline{X})=\mathcal{R}_{2}(\underline{X}), \forall \underline{X} \in D O M_{s m}\left(\widetilde{\mathcal{R}_{1}}\right)
$$

and

$$
D O M^{\mathcal{A}}\left(\widetilde{\mathcal{R}_{1}}\right)=D O M^{\mathcal{A}}\left(\mathcal{R}_{2}\right) \text { and }{\widetilde{\mathcal{R}_{1}}}^{\mathcal{A}}(\underline{\mathfrak{A}})=\mathcal{R}_{2}^{\mathcal{A}}(\underline{\mathfrak{A}}), \forall \underline{\mathfrak{A}} \in D O M^{\mathcal{A}}\left(\widetilde{\mathcal{R}_{1}}\right) .
$$

Therefore, $D O M_{s m}\left(\mathcal{R}_{1}\right) \subseteq D O M_{s m}\left(\widetilde{\mathcal{R}_{1}}\right)=D O M_{s m}\left(\mathcal{R}_{2}\right)$ and $\mathcal{R}_{1}(\underline{X})=\widetilde{\mathcal{R}_{1}}(\underline{X})=$ $\mathcal{R}_{2}(\underline{X})$ for every $\underline{X} \in D O M_{s m}\left(\mathcal{R}_{1}\right)$. Moreover, $D O M^{\mathcal{A}}\left(\mathcal{R}_{1}\right) \subseteq D O M^{\mathcal{A}}\left(\widetilde{\mathcal{R}_{1}}\right)=$ $D O M^{\mathcal{A}}\left(\mathcal{R}_{2}\right)$ and $\mathcal{R}_{1}^{\mathcal{A}}(\underline{\mathfrak{A}})={\widetilde{\mathcal{R}_{1}}}^{\mathcal{A}}(\underline{\mathfrak{A}})=\mathcal{R}_{2}^{\mathcal{A}}(\underline{\mathfrak{A}})$ for every $\underline{\mathfrak{A}} \in D O M^{\mathcal{A}}\left(\mathcal{R}_{1}\right)$.

The following is a summary of all of the main results in this subsection.

Corollary 2.18. If $R$ is a nc rational expression in $x_{1}, \ldots, x_{d}$ over $\mathbb{K}$ and $\underline{Y} \in$ $\operatorname{dom}_{s}(R) \subseteq\left(\mathbb{K}^{s \times s}\right)^{d}$, then $R$ admits a unique (up to unique similarity) minimal $n c$ Fornasini-Marchesini realization centred at $\underline{Y}$ that is also a realization of $R$ w.r.t any unital stably finite $\mathbb{K}$-algebra.

Moreover, any minimal nc Fornasini-Marchesini realization of $R$ centred at $\underline{Y}$ is a realization of $R$ w.r.t any unital stably finite $\mathbb{K}$-algebra.

Proof. From Theorem 2.4, $R$ admits a nc Fornasini-Marchesini realization $\mathcal{R}$ centred at $\underline{Y}$ that is also a nc Fornasini-Marchesini realization of $R$ w.r.t any unital stably finite $\mathbb{K}$-algebra $\mathcal{A}$, while Theorem 2.15 guarantees the existence of a minimal nc Fornasini-Marchesini realization $\widetilde{\mathcal{R}}$ centred at $\underline{Y}$, for which

$$
D O M_{s m}(\mathcal{R}) \subseteq D O M_{s m}(\widetilde{\mathcal{R}}) \text { and } \mathcal{R}(\underline{X})=\widetilde{\mathcal{R}}(\underline{X}), \forall \underline{X} \in D O M_{s m}(\mathcal{R})
$$

for every $m \in \mathbb{N}$, and

$$
D O M^{\mathcal{A}}(\mathcal{R}) \subseteq D O M^{\mathcal{A}}(\widetilde{\mathcal{R}}) \text { and } \mathcal{R}^{\mathcal{A}}(\underline{\mathfrak{A}})=\widetilde{\mathcal{R}}^{\mathcal{A}}(\underline{\mathfrak{A}}), \forall \underline{\mathfrak{A}} \in D O M^{\mathcal{A}}(\mathcal{R}) .
$$

Therefore, for every $m \in \mathbb{N}$,

$$
\operatorname{dom}_{s m}(R) \subseteq D O M_{s m}(\widetilde{\mathcal{R}}) \text { and } R(\underline{X})=\widetilde{\mathcal{R}}(\underline{X}), \forall \underline{X} \in \operatorname{dom}_{s m}(R),
$$

i.e., $\widetilde{\mathcal{R}}$ is a realization of $R$, while the uniqueness of $\widetilde{\mathcal{R}}$ follows from Theorem 2.13.

- Moreover, if $\underline{\mathfrak{a}} \in \operatorname{dom}^{\mathcal{A}}(R)$, then $I_{s} \otimes \underline{\mathfrak{a}} \in D O M^{\mathcal{A}}(\widetilde{\mathcal{R}})$ and $I_{s} \otimes R^{\mathcal{A}}(\underline{\mathfrak{a}})=$ $\mathcal{R}^{\mathcal{A}}\left(I_{s} \otimes \underline{\mathfrak{a}}\right)=\widetilde{\mathcal{R}}^{\mathcal{A}}\left(I_{s} \otimes \underline{\mathfrak{a}}\right)$, i.e., $\widetilde{\mathcal{R}}$ is a realization of $R$ w.r.t $\mathcal{A}$. 
- Furthermore, if $\check{\mathcal{R}}$ is a minimal nc Fornasini-Marchesini realization of $R$ centred at $\underline{Y}$, then $\widetilde{\mathcal{R}}$ and $\check{\mathcal{R}}$ are both minimal nc Fornasini-Marchesini realizations of $R$ centred at $\underline{Y}$, hence by Lemma $2.13, \operatorname{DOM}^{\mathcal{A}}(\check{\mathcal{R}})=\operatorname{DOM}^{\mathcal{A}}(\widetilde{\mathcal{R}})$ and $\check{\mathcal{R}}^{\mathcal{A}}(\underline{\mathfrak{A}})=$ $\widetilde{\mathcal{R}}^{\mathcal{A}}(\underline{\mathfrak{A}})$, for every $\mathfrak{A} \in D O M^{\mathcal{A}}(\check{\mathcal{R}})$. Therefore, for every $\underline{\mathfrak{a}} \in \operatorname{dom}^{\mathcal{A}}(R)$ we have $I_{s} \otimes \underline{\mathfrak{a}} \in D O M^{\mathcal{A}}(\widetilde{\widetilde{\mathcal{R}}})=D O M^{\mathcal{A}}(\check{\mathcal{R}})$ and $I_{s} \otimes R^{\mathcal{A}}(\underline{\mathfrak{a}})=\widetilde{\mathcal{R}}^{\mathcal{A}}\left(I_{s} \otimes \underline{\mathfrak{a}}\right)=\check{\mathcal{R}}^{\mathcal{A}}\left(I_{s} \otimes \underline{\mathfrak{a}}\right)$, i.e., $\check{\mathcal{R}}$ is a nc Fornasini-Marchesini realization of $R$ w.r.t $\mathcal{A}$.

2.5. Example. Consider the nc rational expression $R\left(x_{1}, x_{2}\right)=\left(x_{1} x_{2}-x_{2} x_{1}\right)^{-1}$, with $\mathbb{K}=\mathbb{C}, s=2$ and $\underline{Y}=\left(Y_{1}, Y_{2}\right)=\left(\left(\begin{array}{cc}0 & 1 \\ 1 & 0\end{array}\right),\left(\begin{array}{cc}1 & 0 \\ 0 & -1\end{array}\right)\right) \in \operatorname{dom}_{2}(R)$. We use synthesis and follow the proof of Theorem 2.4, to find a nc Fornasini-Marchesini realization of $R$ centred at $\underline{Y}$ :

- $R_{1}(\underline{x})=x_{1}$ admits a nc Fornasini-Marchesini realization centred at $\underline{Y}$ (see (2.5)), described by

$$
L_{1}=2, D_{1}=\left(\begin{array}{ll}
0 & 1 \\
1 & 0
\end{array}\right), C_{1}=I_{2}, \mathbf{A}_{1}^{1}=\mathbf{A}_{2}^{1}=0_{2}, \mathbf{B}_{1}^{1}=I d_{2}, \mathbf{B}_{2}^{1}=0_{2}
$$

- $R_{2}(\underline{x})=x_{2}$ admits a nc Fornasini-Marchesini realization centred at $\underline{Y}$ (see (2.5)), described by

$$
L_{2}=2, D_{2}=\left(\begin{array}{cc}
1 & 0 \\
0 & -1
\end{array}\right), C_{2}=I_{2}, \mathbf{A}_{1}^{2}=\mathbf{A}_{2}^{2}=0_{2}, \mathbf{B}_{1}^{2}=0_{2}, \mathbf{B}_{2}^{2}=I d_{2}
$$

- $R_{3}(\underline{x})=R_{1}(\underline{x}) R_{2}(\underline{x})=x_{1} x_{2}$ admits a nc Fornasini-Marchesini realization centred at $\underline{Y}$ (see (2.7)), described by

$$
\begin{gathered}
L_{3}=4, D_{3}=\left(\begin{array}{cc}
0 & -1 \\
1 & 0
\end{array}\right), C_{3}=\left(\begin{array}{cccc}
1 & 0 & 0 & 1 \\
0 & 1 & 1 & 0
\end{array}\right), \mathbf{A}_{1}^{3}(X)=\left(I_{2} \otimes X\right)\left[\begin{array}{cc}
0_{2} & I_{2} \\
0_{2} & 0_{2}
\end{array}\right], \\
\mathbf{A}_{2}^{3}(X)=0_{4}, \mathbf{B}_{1}^{3}(X)=\left(I_{2} \otimes X\right)\left[\left(\begin{array}{cc}
1 & 0 \\
0 & -1
\end{array}\right)\right], \mathbf{B}_{2}^{3}(X)=\left(I_{2} \otimes X\right)\left[\begin{array}{l}
0_{2} \\
I_{2}
\end{array}\right] .
\end{gathered}
$$

- $R_{4}(\underline{x})=-R_{2}(\underline{x}) R_{1}(\underline{x})=-x_{2} x_{1}$ admits a nc Fornasini-Marchesini realization centred at $\underline{Y}$ (see (2.7)), described by

$$
\begin{aligned}
& L_{4}=4, D_{4}=\left(\begin{array}{cc}
0 & -1 \\
1 & 0
\end{array}\right), C_{4}=\left(\begin{array}{cccc}
-1 & 0 & -1 & 0 \\
0 & -1 & 0 & 1
\end{array}\right), \mathbf{A}_{1}^{4}(X)=0_{4} \text {, } \\
& \left.\mathbf{A}_{2}^{4}(X)=\left(I_{2} \otimes X\right)\left[\begin{array}{ll}
0_{2} & I_{2} \\
0_{2} & 0_{2}
\end{array}\right], \mathbf{B}_{1}^{4}(X)=\left(I_{2} \otimes X\right)\left[\begin{array}{c}
0_{2} \\
I_{2}
\end{array}\right], \mathbf{B}_{2}^{4}(X)=\left(I_{2} \otimes X\right)\left[\begin{array}{cc}
0 & 1 \\
1 & 0
\end{array}\right)\right] .
\end{aligned}
$$


REALIZATIONS OF NC RATIONAL FUNCTIONS AROUND A MATRIX CENTRE, I 29

- $R_{5}(\underline{x})=R_{3}(\underline{x})+R_{4}(\underline{x})=x_{1} x_{2}-x_{2} x_{1}$ admits a nc Fornasini-Marchesini realization centred at $\underline{Y}$ (see (2.6)), described by

$$
\begin{gathered}
L_{5}=8, D_{5}=\left(\begin{array}{cc}
0 & -2 \\
2 & 0
\end{array}\right), C_{5}=\left(\begin{array}{cccccccc}
1 & 0 & 0 & 1 & -1 & 0 & -1 & 0 \\
0 & 1 & 1 & 0 & 0 & -1 & 0 & 1
\end{array}\right), \\
\mathbf{A}_{1}^{5}(X)=\left(I_{4} \otimes X\right)\left[\begin{array}{cccc}
0_{2} & I_{2} & 0_{2} & 0_{2} \\
0_{2} & 0_{2} & 0_{2} & 0_{2} \\
0_{2} & 0_{2} & 0_{2} & 0_{2} \\
0_{2} & 0_{2} & 0_{2} & 0_{2}
\end{array}\right], \mathbf{A}_{2}(X)=\left(I_{4} \otimes X\right)\left[\begin{array}{llll}
0_{2} & 0_{2} & 0_{2} & 0_{2} \\
0_{2} & 0_{2} & 0_{2} & 0_{2} \\
0_{2} & 0_{2} & 0_{2} & I_{2} \\
0_{2} & 0_{2} & 0_{2} & 0_{2}
\end{array}\right], \\
\mathbf{B}_{1}(X)=\left(I_{4} \otimes X\right)\left[\begin{array}{cc}
1 & 0 \\
0 & -1 \\
0_{2} \\
0_{2} \\
I_{2}
\end{array}\right], \mathbf{B}_{2}(X)=\left(I_{4} \otimes X\right)\left[\begin{array}{cc}
0_{2} \\
I_{2} \\
0 \\
1 & 1 \\
1 & 0 \\
0_{2}
\end{array}\right]
\end{gathered}
$$

- $R(\underline{x})=R_{5}(\underline{x})^{-1}=\left(x_{1} x_{2}-x_{2} x_{1}\right)^{-1}$ admits a nc Fornasini-Marchesini realization $\mathcal{R}$ centred at $\underline{Y}$ (see (2.8)), described by

$$
\begin{aligned}
& L=8, D=\frac{1}{2}\left(\begin{array}{cc}
0 & 1 \\
-1 & 0
\end{array}\right), C=\frac{1}{2}\left(\begin{array}{cccccccc}
0 & 1 & 1 & 0 & 0 & -1 & 0 & 1 \\
-1 & 0 & 0 & -1 & 1 & 0 & 1 & 0
\end{array}\right) \text {, } \\
& \mathbf{A}_{1}(X)=\frac{1}{2}\left(I_{4} \otimes X\right)\left[\begin{array}{cccc}
\left(\begin{array}{cc}
0 & -1 \\
-1 & 0
\end{array}\right) & I_{2} & \left(\begin{array}{cc}
0 & 1 \\
1 & 0
\end{array}\right) & \left(\begin{array}{cc}
0 & -1 \\
1 & 0
\end{array}\right) \\
0_{2} & 0_{2} & 0_{2} & 0_{2} \\
0_{2} & 0_{2} & 0_{2} & 0_{2} \\
\left(\begin{array}{cc}
0 & -1 \\
1 & 0
\end{array}\right) & \left(\begin{array}{cc}
-1 & 0 \\
0 & 1
\end{array}\right) & \left(\begin{array}{cc}
0 & 1 \\
-1 & 0
\end{array}\right) & \left(\begin{array}{cc}
0 & -1 \\
-1 & 0
\end{array}\right)
\end{array}\right] \text {, }
\end{aligned}
$$

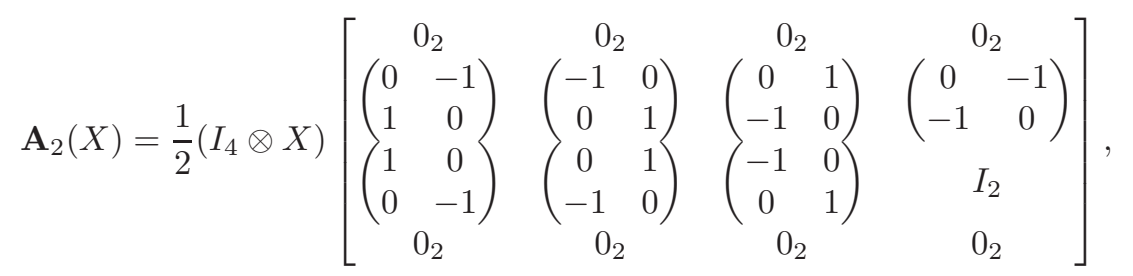

$$
\begin{aligned}
& \mathbf{B}_{1}(X)=\frac{1}{2}\left(I_{4} \otimes X\right)\left[\begin{array}{c}
\left(\begin{array}{cc}
0 & -1 \\
-1 & 0
\end{array}\right) \\
0_{2} \\
0_{2} \\
\left(\begin{array}{cc}
0 & -1 \\
1 & 0
\end{array}\right)
\end{array}\right], \mathbf{B}_{2}(X)=\frac{1}{2}\left(I_{4} \otimes X\right)\left[\begin{array}{c}
0_{2} \\
\left(\begin{array}{cc}
0 & -1 \\
1 & 0 \\
1 & 0 \\
0 & -1
\end{array}\right) \\
0_{2}
\end{array}\right] .
\end{aligned}
$$

The nc Fornasini-Marchesini realization $\mathcal{R}$ is controllable, however $\mathcal{R}$ is not observable, as

$$
\mathcal{N O} \mathcal{O}_{C, \underline{\mathbf{A}}}=\bigcap_{\omega \in \mathcal{G}_{2}, Z_{1}, \ldots, Z_{\ell} \in \mathbb{C}^{2 \times 2}} \operatorname{ker}\left(C \underline{\mathbf{A}}^{\omega}\left(Z_{1}, \ldots, Z_{\ell}\right)\right)=\operatorname{span}\left\{\underline{e}_{1}+\underline{e}_{5}, \underline{e}_{2}+\underline{e}_{6}\right\},
$$

hence $\mathcal{R}$ is not minimal. By the Kalman decomposition (see Theorem 2.15) argument, we obtain a minimal nc Fornasini-Marchesini realization of $R$ centred at $\underline{Y}$ 
of dimension $\widetilde{L}=\operatorname{dim}\left(\mathcal{C}_{\underline{\mathbf{A}}, \underline{\mathbf{B}}}\right)-\operatorname{dim}\left(\mathcal{N} \mathcal{O}_{C, \underline{\mathbf{A}}} \cap \mathcal{C}_{\underline{\mathbf{A}}, \underline{\mathbf{B}}}\right)=6$, that is

$\widetilde{\mathcal{R}}\left(X_{1}, X_{2}\right)=\widetilde{D}+\widetilde{C}\left(I_{6}-\widetilde{\mathbf{A}_{1}}\left(X_{1}-Y_{1}\right)-\widetilde{\mathbf{A}_{2}}\left(X_{2}-Y_{2}\right)\right)^{-1}\left(\widetilde{\mathbf{B}_{1}}\left(X_{1}-Y_{1}\right)+\widetilde{\mathbf{B}_{2}}\left(X_{2}-Y_{2}\right)\right)$,

with

$$
\begin{aligned}
& \widetilde{D}=\frac{1}{2}\left(\begin{array}{cc}
0 & 1 \\
-1 & 0
\end{array}\right), \widetilde{C}=\frac{1}{2}\left(\begin{array}{cccccc}
1 & 0 & 0 & 1 & 0 & 2 \\
0 & -1 & 1 & 0 & -2 & 0
\end{array}\right), \\
& \widetilde{\mathbf{A}_{1}}(X)=\frac{1}{2}\left(I_{3} \otimes X\right)\left[\begin{array}{ccc}
0_{2} & 0_{2} & 0_{2} \\
\left(\begin{array}{cc}
-1 & 0 \\
0 & 1
\end{array}\right) & \left(\begin{array}{cc}
0 & -1 \\
-1 & 0
\end{array}\right) & \left(\begin{array}{cc}
0 & -2 \\
2 & 0
\end{array}\right) \\
\left(\begin{array}{cc}
\frac{1}{2} & 0 \\
0 & \frac{1}{2}
\end{array}\right) & \left(\begin{array}{cc}
0 & -\frac{1}{2} \\
\frac{1}{2} & 0
\end{array}\right) & \left(\begin{array}{cc}
0 & -1 \\
-1 & 0
\end{array}\right)
\end{array}\right],
\end{aligned}
$$

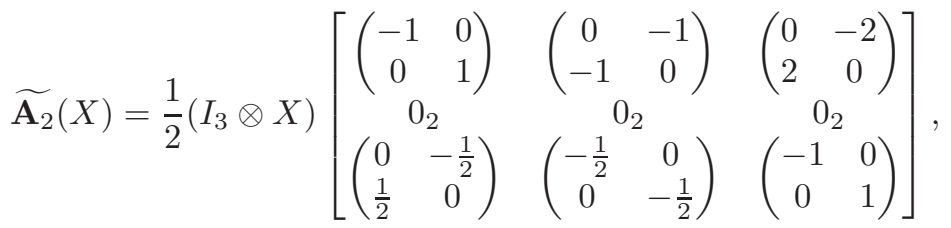

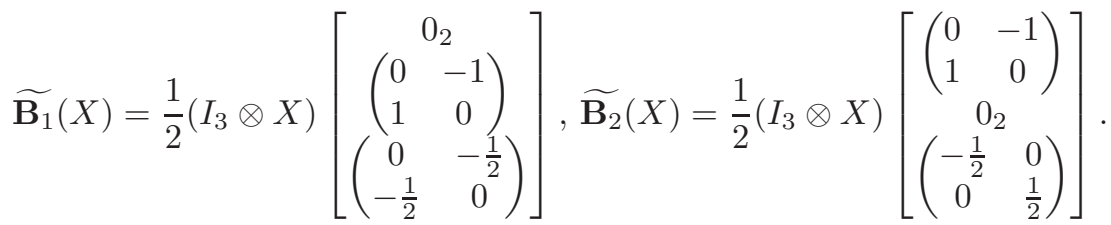

In this example it is easy to check directly that

$$
\operatorname{det}\left(I_{6 m}-\sum_{k=1}^{2}\left(X_{k}-I_{m} \otimes Y_{k}\right) \widetilde{\mathbf{A}_{k}}\right) \neq 0 \Longleftrightarrow \operatorname{det}\left(X_{1} X_{2}-X_{2} X_{1}\right) \neq 0
$$

for $\underline{X}=\left(X_{1}, X_{2}\right) \in\left(\mathbb{C}^{2 m \times 2 m}\right)^{2}$, i.e., that $\operatorname{dom}_{2 m}(R)=D O M_{2 m}(\widetilde{\mathcal{R}})$ and also that $R(\underline{X})=\widetilde{\mathcal{R}}(\underline{X})$. Furthermore, for any unital stably finite $\mathbb{C}$-algebra $\mathcal{A}$ and $\underline{\mathfrak{a}}=\left(\mathfrak{a}_{1}, \mathfrak{a}_{2}\right) \in \mathcal{A}^{2}$, the element $\mathfrak{a}_{1} \mathfrak{a}_{2}-\mathfrak{a}_{2} \mathfrak{a}_{1}$ is invertible in $\mathcal{A}$ if and only if the matrix

$$
\left(I_{6} \otimes 1_{\mathcal{A}}-\sum_{k=1}^{2} \widetilde{\mathbf{A}_{k}}\left(I_{2}\right) \otimes \mathfrak{a}_{k}-\widetilde{\mathbf{A}_{k}}\left(Y_{k}\right) \otimes 1_{\mathcal{A}}\right) \text { is invertible in } \mathcal{A}^{6 \times 6}
$$

i.e., $\operatorname{dom}^{\mathcal{A}}(R)=D O M^{\mathcal{A}}(\widetilde{\mathcal{R}})$ and also $I_{2} \otimes R^{\mathcal{A}}(\underline{\mathfrak{a}})=\mathcal{R}^{\mathcal{A}}\left(I_{2} \otimes \underline{\mathfrak{a}}\right)$.

2.6. Cohn's theorem. As a corollary of the results in Subsection 2.4, we get a new proof of a theorem of Cohn, stating that if two nc rational expressions represents the same nc rational function, then they are $\mathcal{A}$-evaluation equivalent for any unital stably finite $\mathbb{K}$-algebra $\mathcal{A}$. Cohn's Theorem was proved originally in [24] and afterwards in his book [25, Theorem 7.3.2]. In [41], the authors proved a weaker version of the theorem, applicable only for nc rational expressions which are regular at the origin, using their theory of realizations for nc regular rational functions. We omit their assumption on regularity at the origin and prove the theorem in its full version.

Theorem 2.19 (Cohn's Theorem). Let $R$ and $\widetilde{R}$ be $\left(\mathbb{K}^{d}\right)_{n c}$-evaluation equivalent nc rational expressions in $x_{1}, \ldots, x_{d}$ over $\mathbb{K}$, i.e., $R(\underline{X})=\widetilde{R}(\underline{X})$ for all 
$\underline{X} \in \operatorname{dom}(R) \cap \operatorname{dom}(\widetilde{R})$. Then $R$ and $\widetilde{R}$ are $\mathcal{A}$-evaluation equivalent for any unital stably finite $\mathbb{K}$-algebra $\mathcal{A}$, i.e.,

$$
R^{\mathcal{A}}(\underline{\mathfrak{a}})=\widetilde{R}^{\mathcal{A}}(\underline{\mathfrak{a}}), \forall \underline{\mathfrak{a}} \in \operatorname{dom}^{\mathcal{A}}(R) \cap \operatorname{dom}^{\mathcal{A}}(\widetilde{R}) .
$$

Proof. Assume $R$ and $\widetilde{R}$ are non-degenerate nc rational expressions, so there exists $s \in \mathbb{N}$ such that $\operatorname{dom}_{s}(R), \operatorname{dom}_{s}(\widetilde{R}) \neq \emptyset$. As $\operatorname{dom}_{s}(R), \operatorname{dom}_{s}(\widetilde{R})$ are Zariski open sets in $\mathbb{K}^{s \times s}$, there exists $\ell \in \mathbb{N}$ such that $\operatorname{dom}_{s \ell}(R) \cap \operatorname{dom}_{s \ell}(\widetilde{R}) \neq \emptyset$. The reasoning for that is clear when $\mathbb{K}$ is infinite (with $\ell=1$ ), while if $\mathbb{K}$ is finite we use a similar argument as in [48, Remark 2.6]. Let

$$
\underline{Y}=\left(Y_{1}, \ldots, Y_{d}\right) \in \operatorname{dom}_{s \ell}(R) \cap \operatorname{dom}_{s \ell}(\widetilde{R}) .
$$

From Corollary 2.18, $R$ and $\widetilde{R}$ admit minimal nc Fornasini-Marchesini realizations $\mathcal{R}$ and $\widetilde{\mathcal{R}}$, respectively, both centred at $\underline{Y}$ with the special properties:

$$
\begin{aligned}
& \underline{\mathfrak{a}} \in \operatorname{dom}^{\mathcal{A}}(R) \Longrightarrow I_{s \ell} \otimes \underline{\mathfrak{a}} \in D O M^{\mathcal{A}}(\mathcal{R}) \text { and } I_{s \ell} \otimes R^{\mathcal{A}}(\underline{\mathfrak{a}})=\mathcal{R}^{\mathcal{A}}\left(I_{s \ell} \otimes \underline{\mathfrak{a}}\right), \\
& \underline{\mathfrak{a}} \in \operatorname{dom}^{\mathcal{A}}(\widetilde{R}) \Longrightarrow I_{s \ell} \otimes \underline{\mathfrak{a}} \in D O M^{\mathcal{A}}(\widetilde{\mathcal{R}}) \text { and } I_{s \ell} \otimes \widetilde{R}^{\mathcal{A}}(\underline{\mathfrak{a}})=\widetilde{\mathcal{R}}^{\mathcal{A}}\left(I_{s \ell} \otimes \underline{\mathfrak{a}}\right) .
\end{aligned}
$$

Moreover, Theorem 2.13 implies that

$$
D O M^{\mathcal{A}}(\mathcal{R})=D O M^{\mathcal{A}}(\widetilde{\mathcal{R}}) \text { and } \mathcal{R}^{\mathcal{A}}(\underline{\mathfrak{A}})=\widetilde{\mathcal{R}}^{\mathcal{A}}(\underline{\mathfrak{A}}), \forall \underline{\mathfrak{A}} \in D O M^{\mathcal{A}}(\mathcal{R}) .
$$

Finally, if

$$
\underline{\mathfrak{a}} \in \operatorname{dom}^{\mathcal{A}}(R) \cap \operatorname{dom}^{\mathcal{A}}(\widetilde{R}),
$$

then $I_{s \ell} \otimes \underline{\mathfrak{a}} \in D O M^{\mathcal{A}}(\mathcal{R})=D O M^{\mathcal{A}}(\widetilde{\mathcal{R}})$ and $I_{s \ell} \otimes R^{\mathcal{A}}(\underline{\mathfrak{a}})=\mathcal{R}^{\mathcal{A}}\left(I_{s \ell} \otimes \underline{\mathfrak{a}}\right)=$ $\widetilde{\mathcal{R}}^{\mathcal{A}}\left(I_{s \ell} \otimes \underline{\mathfrak{a}}\right)=I_{s \ell} \otimes \widetilde{R}^{\mathcal{A}}(\underline{\mathfrak{a}})$, therefore $R^{\mathcal{A}}(\underline{\mathfrak{a}})=\widetilde{R}^{\mathcal{A}}(\underline{\mathfrak{a}})$.

Remark 2.20. Theorem 2.19 implies that one can evaluate any nc rational function by evaluating a minimal realization of the function. This proves that $\mathbb{K} \nless \underline{x} \ngtr$ is the universal skew field of fractions of $\mathbb{K}\langle\underline{x}\rangle$, see $[21,66]$ for the original proofs and [48] for a modern proof. We postpone a detailed discussion and an application to an explicit construction of $\mathbb{K} \nless \underline{x} \ngtr$ to [64].

2.7. The McMillan degree. For a nc rational expression $R$ in $x_{1}, \ldots, x_{d}$ over $\mathbb{K}$ and $\underline{Y} \in \operatorname{dom}(R)$, we define by $L_{R}(\underline{Y})$ the dimension of a minimal nc FornasiniMarchesini realization of $R$ centred at $\underline{Y}$. The first part of the next theorem is an analog of [82, Theorem 5.10].

Theorem 2.21. Let $R$ be a non-degenerate nc rational expression in $x_{1}, \ldots, x_{d}$ over $\mathbb{K}$ and let $\underline{Y} \in \operatorname{dom}_{s}(R)$.

1. If $\underline{\tilde{Y}} \in \operatorname{dom}_{s}(R)$, then $L_{R}(\underline{Y})=L_{R}(\underline{\tilde{Y}})$.

2. If $n \in \mathbb{N}$, then $I_{n} \otimes \underline{Y} \in \operatorname{dom}_{s n}(R)$ and $L_{R}\left(I_{n} \otimes \underline{Y}\right)=n L_{R}(\underline{Y})$.

3. If $s^{\prime} \in \mathbb{N}$ and $\underline{Y}^{\prime} \in \operatorname{dom}_{s^{\prime}}(R)$, then $s^{\prime} L_{R}(\underline{Y})=s L_{R}\left(\underline{Y}^{\prime}\right)$.

Proof. Applying Corollary 2.18, the expression $R$ admits a minimal nc FornasiniMarchesini realization $\mathcal{R}$ centred at $\underline{Y}$, described by a tuple $(L, D, C, \underline{\mathbf{A}}, \underline{\mathbf{B}})$. Let

$$
T_{1}:=I_{L}-\sum_{k=1}^{d} \mathbf{A}_{k}\left(\widetilde{Y_{k}}-Y_{k}\right) \text { and } T_{2}:=\sum_{k=1}^{d} \mathbf{B}_{k}\left(Y_{k}-\widetilde{Y_{k}}\right),
$$


as $\underline{\underline{Y}}=\left(\widetilde{Y_{1}}, \ldots, \widetilde{Y_{d}}\right) \in \operatorname{dom}_{s}(R) \subseteq D O M_{s}(\mathcal{R})$, the matrix $T_{1}$ is invertible. Therefore, for every $\underline{X} \in D O M_{s m}(\mathcal{R})$ and $m \in \mathbb{N}$ :

$$
\begin{aligned}
& \mathcal{R}(\underline{X})=I_{m} \otimes D+\left(I_{m} \otimes C\right)\left(I_{L m}-\sum_{k=1}^{d}\left(X_{k}-I_{m} \otimes \widetilde{Y_{k}}\right) \mathbf{A}_{k}-\sum_{k=1}^{d}\left(I_{m} \otimes\left(\widetilde{Y_{k}}-Y_{k}\right)\right) \mathbf{A}_{k}\right)^{-1}\left(\sum_{k=1}^{d}\right. \\
& \left.\left(X_{k}-I_{m} \otimes \widetilde{Y_{k}}\right) \mathbf{B}_{k}-\sum_{k=1}^{d}\left(I_{m} \otimes\left(Y_{k}-\widetilde{Y_{k}}\right)\right) \mathbf{B}_{k}\right)=I_{m} \otimes D+\left(I_{m} \otimes C T_{1}^{-1}\right)\left(I_{L m}-\sum_{k=1}^{d}\left(X_{k}-I_{m} \otimes \widetilde{Y_{k}}\right) \widetilde{\mathbf{A}_{k}}\right)^{-1} \\
& \sum_{k=1}^{d}\left(X_{k}-I_{m} \otimes \widetilde{Y_{k}}\right) \mathbf{B}_{k}-\left(I_{m} \otimes C T_{1}^{-1}\right)\left(I_{L m}-\sum_{k=1}^{d}\left(X_{k}-I_{m} \otimes \widetilde{Y_{k}}\right) \widetilde{\mathbf{A}_{k}}\right)^{-1}\left(I_{m} \otimes T_{2}\right),
\end{aligned}
$$

where $\widetilde{\mathbf{A}_{k}}=\mathbf{A}_{k} \cdot T_{1}^{-1}$, and since

$$
\begin{array}{r}
\left(I_{L m}-\sum_{k=1}^{d}\left(X_{k}-I_{m} \otimes \widetilde{Y_{k}}\right) \widetilde{\mathbf{A}_{k}}\right)^{-1}\left(I_{m} \otimes T_{2}\right)=I_{m} \otimes T_{2}+\left(I_{L m}-\sum_{k=1}^{d}\left(X_{k}-I_{m} \otimes \widetilde{Y_{k}}\right) \widetilde{\mathbf{A}_{k}}\right)^{-1} \\
\sum_{k=1}^{d}\left(X_{k}-I_{m} \otimes \widetilde{Y_{k}}\right) \widehat{\mathbf{B}_{k}},
\end{array}
$$

where $\widehat{\mathbf{B}_{k}}=\widetilde{\mathbf{A}_{k}} \cdot T_{2}$, we conclude that

$$
\begin{gathered}
\mathcal{R}(\underline{X})=I_{m} \otimes D-I_{m} \otimes\left(C T_{1}^{-1} T_{2}\right)+\left(I_{m} \otimes C T_{1}^{-1}\right)\left(I_{L m}-\sum_{k=1}^{d}\left(X_{k}-I_{m} \otimes \widetilde{Y_{k}}\right) \widetilde{\mathbf{A}_{k}}\right)^{-1} \\
\sum_{k=1}^{d}\left(X_{k}-I_{m} \otimes \widetilde{Y_{k}}\right) \mathbf{B}_{k}-\left(I_{m} \otimes C T_{1}^{-1}\right)\left(I_{L m}-\sum_{k=1}^{d}\left(X_{k}-I_{m} \otimes \widetilde{Y_{k}}\right) \widetilde{\mathbf{A}_{k}}\right)^{-1} \sum_{k=1}^{d}\left(X_{k}-I_{m} \otimes \widetilde{Y_{k}}\right) \widehat{\mathbf{B}_{k}}
\end{gathered}
$$

i.e., that $\mathcal{R}(\underline{X})=\widetilde{\mathcal{R}}(\underline{X})$ where $\widetilde{\mathcal{R}}$ is a nc Fornasini-Marchesini realization of $R$, centred at $\underline{\underline{Y}}$, described by

$$
\widetilde{D}=D-C T_{1}^{-1} T_{2}, \widetilde{C}=C T_{1}^{-1}, \widetilde{\mathbf{A}_{k}}=\mathbf{A}_{k} \cdot T_{1}^{-1}, \widetilde{\mathbf{B}_{k}}=\mathbf{B}_{k}-\mathbf{A}_{k} \cdot\left(T_{1}^{-1} T_{2}\right) .
$$

Thus, $L_{R}(\underline{\tilde{Y}}) \leq L=L_{R}(\underline{Y})$ and by symmetry we get that $L_{R}(\underline{\tilde{Y}})=L_{R}(\underline{Y})$, hence $\widetilde{\mathcal{R}}$ is a minimal nc Fornasini-Marchesini realization of $R$ centred at $\underline{\underline{Y}}$.

- Suppose next that $n \in \mathbb{N}$. As $\operatorname{dom}(R)$ is closed under direct sums it follows that $I_{n} \otimes \underline{Y} \in \operatorname{dom}_{s n}(R)$, while for every $p \in \mathbb{N}$ letting $m=n p$ yields for every $\underline{X} \in \operatorname{dom}_{\text {snp }}(R):$

$$
\begin{aligned}
& \mathcal{R}(\underline{X})=I_{n p} \otimes D+\left(I_{n p} \otimes C\right)\left(I_{L n p}-\sum_{k=1}^{d}\left(X_{k}-I_{n p} \otimes Y_{k}\right) \mathbf{A}_{k}\right)^{-1} \sum_{k=1}^{d}\left(X_{k}-I_{n p} \otimes Y_{k}\right) \mathbf{B}_{k} \\
= & I_{p} \otimes D^{(n)}+\left(I_{p} \otimes C^{(n)}\right)\left(I_{L^{(n)} p}-\sum_{k=1}^{d}\left(X_{k}-I_{p} \otimes Y_{k}^{(n)}\right) \mathbf{A}_{k}\right)^{-1} \sum_{k=1}^{d}\left(X_{k}-I_{p} \otimes Y_{k}^{(n)}\right) \mathbf{B}_{k}
\end{aligned}
$$

where

$$
L^{(n)}=L n, D^{(n)}=I_{n} \otimes D, C^{(n)}=I_{n} \otimes C \text { and } Y_{k}^{(n)}=I_{n} \otimes Y_{k} .
$$

We obtained a nc Fornasini-Marchesini realization $\mathcal{R}^{(n)}$ — described by the tuple $\left(L^{(n)}, D^{(n)}, C^{(n)}, \underline{\mathbf{A}}, \underline{\mathbf{B}}\right)$ - of $R$ that is centred at $\underline{Y}^{(n)}:=I_{n} \otimes \underline{Y}$ and it is easily 
seen that controllability and observability of $\mathcal{R}$ imply the controllability and observability of $\mathcal{R}^{(n)}$ as well, thus $\mathcal{R}^{(n)}$ is minimal and hence $L_{R}\left(I_{n} \otimes \underline{Y}\right)=n L_{R}(\underline{Y})$.

- Finally, let $\underline{Y^{\prime}} \in \operatorname{dom}_{s^{\prime}}(R)$, then part 2 implies that $I_{s^{\prime}} \otimes \underline{Y}, I_{s} \otimes \underline{Y^{\prime}} \in \operatorname{dom}_{s s^{\prime}}(R)$, $L_{R}\left(I_{s^{\prime}} \otimes \underline{Y}\right)=s^{\prime} L_{R}(\underline{Y})$ and $L_{R}\left(I_{s} \otimes \underline{Y}^{\prime}\right)=s L_{R}\left(\underline{Y}^{\prime}\right)$, while from part 1, $L_{R}\left(I_{s^{\prime}} \otimes\right.$ $\underline{Y})=L_{R}\left(I_{s} \otimes \underline{Y}^{\prime}\right)$, therefore $s^{\prime} L_{R}(\underline{Y})=s L_{R}\left(\underline{Y}^{\prime}\right)$.

Remark 2.22. In the proof of Theorem 2.21 we built explicit minimal nc FornasiniMarchesini realizations $\widetilde{\mathcal{R}}$ and $\mathcal{R}^{(n)}$ of $R$, centred at $\underline{\underline{Y}}$ and $I_{n} \otimes \underline{Y}$, respectively, using a minimal realization $\mathcal{R}$ of $R$ centred at $\underline{Y}$. From Corollary 2.18 it follows right away that $\widetilde{\mathcal{R}}$ and $\mathcal{R}^{(n)}$ are also nc Fornasini-Marchesini realizations of $R$ w.r.t any unital stably finite $\mathbb{K}$-algebra $\mathcal{A}$. Moreover, direct computations-which are omitted - easily show that

$$
D O M^{\mathcal{A}}(\mathcal{R})=D O M^{\mathcal{A}}(\widetilde{\mathcal{R}}) \text { and } \mathcal{R}^{\mathcal{A}}(\underline{\mathfrak{A}})=\widetilde{\mathcal{R}}^{\mathcal{A}}(\underline{\mathfrak{A}}), \forall \underline{\mathfrak{A}} \in D O M^{\mathcal{A}}(\mathcal{R})
$$

and

$\underline{\mathfrak{A}} \in D O M^{\mathcal{A}}(\mathcal{R}) \Longleftrightarrow I_{n} \otimes \underline{\mathfrak{A}} \in D O M^{\mathcal{A}}\left(\mathcal{R}^{(n)}\right)$ and $I_{n} \otimes \mathcal{R}^{\mathcal{A}}(\underline{\mathfrak{A}})=\left(\mathcal{R}^{(n)}\right)^{\mathcal{A}}\left(I_{n} \otimes \underline{\mathfrak{A}}\right)$.

The first part of Theorem 2.21 guarantees that the value $L_{R}(\underline{Y})$ does not depend on $\underline{Y}$ but only on $s$, so it will be denoted $L_{R}(s):=L_{R}(\underline{Y})$, while from the third part of the theorem it follows that there exists $\mathfrak{m}(R)>0$ such that

$$
L_{R}(s)=\mathfrak{m}(R) s, \quad \forall s \geq 1
$$

where $\mathfrak{m}(R)$ depends only on $R$; We define $\mathfrak{m}(R)$ as the McMillan degree of $R$.

In the next lemma we actually show that $\mathfrak{m}(R) \in \mathbb{N}$. This is a direct corollary and yet separated from the arguments of Theorem 2.21, as it requires a non-trivial tool from PI-ring theory, that is if $R$ is a non-degenerate nc rational expression, then there exists $n \in \mathbb{N}$ such that $\operatorname{dom}_{k}(R) \neq \emptyset$ for every $k \geq n$; see [66, Chapter $8]$ and [49, Remarks 2.15 and 2.16] for a more detailed discussion.

Lemma 2.23. If $R$ is a non-degenerate nc rational expression in $x_{1}, \ldots, x_{d}$ over $\mathbb{K}$ and $\operatorname{dom}_{s}(R) \neq \emptyset$, then $s \mid L_{R}(s)$ and hence $\mathfrak{m}(R) \in \mathbb{N}$.

Proof. As $\operatorname{dom}_{s}(R) \neq \emptyset$, let $\underline{Y} \in \operatorname{dom}_{s}(R)$ and according to Corollary 2.18, let $\mathcal{R}$ be a minimal nc Fornasini-Marchesini realization of $R$, centred at $\underline{Y}$. Since $R$ is non-degenerate, there exists $n \in \mathbb{N}$ such that $\operatorname{dom}_{k}(R) \neq \emptyset$ for all $k \geq n$. Consider the sequence $\left(k_{j}\right)_{j \geq 1}$ given by $k_{j}=s j+1$, clearly for $j$ large enough we get $k_{j} \geq n$ and hence $\operatorname{dom}_{k_{j}}(R) \neq \emptyset$. Let $\underline{W} \in \operatorname{dom}_{k_{j}}(R)$ and apply Theorem 2.21 for $\underline{W}$ and $\underline{Y}$; we obtain that $k_{j} L_{R}(\underline{Y})=s L_{R}(\underline{W})$, but it is easily seen that $s$ and $k_{j}$ are co-prime integers, thus $s \mid L_{R}(\underline{Y})$ and hence $\mathfrak{m}(R) \in \mathbb{N}$.

Remark 2.24. If $R$ is a nc rational expression in $x_{1}, \ldots, x_{d}$ over $\mathbb{K}, \underline{Y}_{1} \in$ dom $_{s_{1}}(R)$ and $\underline{Y}_{2} \in \operatorname{dom}_{s_{2}}(R)$, then $\underline{Y}_{1} \oplus \underline{Y}_{2} \in d o m_{s_{1}+s_{2}}(R)$ and (2.25) implies that

$$
L_{R}\left(s_{1}+s_{2}\right)=\left(s_{1}+s_{2}\right) \mathfrak{m}(R)=s_{1} \mathfrak{m}(R)+s_{2} \mathfrak{m}(R)=L_{R}\left(s_{1}\right)+L_{R}\left(s_{2}\right) .
$$

If $R$ admits two minimal nc Fornasini-Marchesini realizations $\mathcal{R}_{1}$ and $\mathcal{R}_{2}$, centred at $\underline{Y}_{1}$ and $\underline{Y}_{2}$, respectively, which are described by the tuples $\left(L_{1}, D^{1}, C^{1}, \underline{\mathbf{A}}^{1}, \underline{\mathbf{B}}^{1}\right)$ and $\left(L_{2}, D^{2}, C^{2}, \underline{\mathbf{A}}^{2}, \underline{\mathbf{B}}^{2}\right)$, it is very tempting to consider $\mathcal{R}$ to be a nc FornasiniMarchesini realization of $R$ centred at $\underline{Y}_{1} \oplus \underline{Y}_{2}$, where $\mathcal{R}$ is described by

$$
D=D^{1} \oplus D^{2}, C=C^{1} \oplus C^{2}, \mathbf{A}_{k}=\left[\begin{array}{cc}
\mathbf{A}_{k}^{1} & 0 \\
0 & \mathbf{A}_{k}^{2}
\end{array}\right] \text { and } \mathbf{B}_{k}=\left[\begin{array}{l}
\mathbf{B}_{k}^{1} \\
\mathbf{B}_{k}^{2}
\end{array}\right], 1 \leq k \leq d
$$


but we only know that $R(\underline{X})=\mathcal{R}(\underline{X})$ whenever $\underline{X} \in$ dom $_{s_{1}+s_{2}}(R)$ is of the form $\underline{X}=\underline{X}^{(1)} \oplus \underline{X}^{(2)}$, with $\underline{X}^{(1)} \in \operatorname{dom}_{s_{1}}\left(R_{1}\right)$ and $\underline{X}^{\overline{(2)}} \in \operatorname{dom}_{s_{2}}\left(R_{2}\right)$.

\section{Realizations of NC Rational Functions}

From the previous section (cf. Theorem 2.13) we know that - given a nc rational function - all of its minimal nc Fornasini-Marchesini realizations which are centred at the same point, must have the same domain (and $\mathcal{A}$-domain) and same evaluation (w.r.t $\mathcal{A}$ as well; here $\mathcal{A}$ is a unital stably finite $\mathbb{K}$-algebra).

In this section we continue to establish connections between all minimal nc Fornasini-Marchesini realizations (with centres of all possible sizes) of a given nc rational function. Using Lemma 2.21 and Remark 2.22, the general case - where the two centres of minimal realizations of a rational function are different- is considered and solved, which then will lead us to the main conclusion, that is Theorem 3.3.

Lemma 3.1. Let $R_{1}$ and $R_{2}$ be nc rational expressions in $x_{1}, \ldots, x_{d}$ over $\mathbb{K}$, with $\underline{Y}_{1} \in \operatorname{dom}_{s_{1}}\left(R_{1}\right)$ and $\underline{Y}_{2} \in \operatorname{dom}_{s_{2}}\left(R_{2}\right)$, where $s_{1}, s_{2} \in \mathbb{N}$. Suppose $\mathcal{R}_{1}$ and $\mathcal{R}_{2}$ are minimal nc Fornasini-Marchesini realizations of $R_{1}$ and $R_{2}$, centred at $\underline{Y}_{1}$ and $\underline{Y}_{2}$, respectively. If $R_{1}$ and $R_{2}$ are $\left(\mathbb{K}^{d}\right)_{n c}$-evaluation equivalent, then

$$
D O M_{p m}\left(\mathcal{R}_{1}\right)=D O M_{p m}\left(\mathcal{R}_{2}\right) \text { and } \mathcal{R}_{1}(\underline{X})=\mathcal{R}_{2}(\underline{X})
$$

for every $m \in \mathbb{N}$ and $\underline{X} \in D O M_{p m}\left(\mathcal{R}_{1}\right)$, where $p=$ l.c.m $\left(s_{1}, s_{2}\right)$. Moreover, for any unital stably finite $\mathbb{K}$-algebra $\mathcal{A}$ and $\underline{\mathfrak{a}} \in \mathcal{A}^{d}$ :

$$
I_{s_{1}} \otimes \underline{\mathfrak{a}} \in D O M^{\mathcal{A}}\left(\mathcal{R}_{1}\right) \Longleftrightarrow I_{s_{2}} \otimes \underline{\mathfrak{a}} \in D O M^{\mathcal{A}}\left(\mathcal{R}_{2}\right)
$$

and for every such $\underline{\mathfrak{a}}$, we have

$$
I_{s_{2}} \otimes \mathcal{R}_{1}^{\mathcal{A}}\left(I_{s_{1}} \otimes \underline{\mathfrak{a}}\right)=I_{s_{1}} \otimes \mathcal{R}_{2}^{\mathcal{A}}\left(I_{s_{2}} \otimes \underline{\mathfrak{a}}\right) .
$$

Proof. We know that $\operatorname{dom}_{s_{1}}\left(R_{1}\right), \operatorname{dom}_{s_{2}}\left(R_{2}\right) \neq \emptyset$, hence $\operatorname{dom}_{p}\left(R_{1}\right), \operatorname{dom}_{p}\left(R_{2}\right) \neq \emptyset$ and as they are both open Zariski sets in $\left(\mathbb{K}^{p \times p}\right)^{d}$, there exists $\ell \in \mathbb{N}$ such that $\operatorname{dom}_{\ell p}\left(R_{1}\right) \cap \operatorname{dom}_{\ell p}\left(R_{2}\right) \neq \emptyset$, so let us fix

$$
\underline{\tilde{Y}} \in \operatorname{dom}_{\ell p}\left(R_{1}\right) \cap \operatorname{dom}_{\ell p}\left(R_{2}\right) \subseteq D O M_{\ell p}\left(\mathcal{R}_{1}\right) \cap D O M_{\ell p}\left(\mathcal{R}_{2}\right) .
$$

Once again (as in the proof of Theorem 2.19), the reasoning for that is clear when $\mathbb{K}$ is infinite (with $\ell=1$ ), while if $\mathbb{K}$ is finite we use a similar argument as in [48, Remark 2.6].

- Let $n_{1}$ and $n_{2}$ be the integers for which $p \ell=s_{1} n_{1}=s_{2} n_{2}$. From Remark 2.22, there exist minimal nc Fornasini-Marchesini realizations $\mathcal{R}^{\left(n_{k}\right)}$ of $R_{k}$, centred at $I_{n_{k}} \otimes \underline{Y}_{k}$, such that

$$
D O M_{p \ell m}\left(\mathcal{R}_{k}\right)=D O M_{p \ell m}\left(\mathcal{R}^{\left(n_{k}\right)}\right) \text { and } \mathcal{R}^{\left(n_{k}\right)}(\underline{X})=\mathcal{R}_{k}(\underline{X}), \forall \underline{X} \in D O M_{p \ell m}\left(\mathcal{R}_{k}\right)
$$

for every $m \in \mathbb{N}, \underline{\mathfrak{A}} \in D O M^{\mathcal{A}}\left(\mathcal{R}_{k}\right) \Longleftrightarrow I_{n_{k}} \otimes \underline{\mathfrak{A}} \in D O M^{\mathcal{A}}\left(\mathcal{R}^{\left(n_{k}\right)}\right)$ and

$$
\left(\mathcal{R}^{\left(n_{k}\right)}\right)^{\mathcal{A}}\left(I_{n_{k}} \otimes \underline{\mathfrak{A}}\right)=I_{n_{k}} \otimes \mathcal{R}_{k}^{\mathcal{A}}(\underline{\mathfrak{A}}), \forall \underline{\mathfrak{A}} \in \operatorname{DOM}^{\mathcal{A}}\left(\mathcal{R}_{k}\right),
$$

for $k=1,2$. In addition, there exist minimal nc Fornasini-Marchesini realizations $\widetilde{\mathcal{R}_{k}}$ of $R_{k}$, centred at $\underline{\tilde{Y}}$, such that

$$
D O M_{p \ell m}\left(\mathcal{R}^{\left(n_{k}\right)}\right)=D O M_{p \ell m}\left(\widetilde{\mathcal{R}_{k}}\right) \text { and } \mathcal{R}^{\left(n_{k}\right)}(\underline{X})=\widetilde{\mathcal{R}_{k}}(\underline{X}), \forall \underline{X} \in D O M_{p \ell m}\left(\widetilde{\mathcal{R}_{k}}\right)
$$


for every $m \in \mathbb{N}, D O M^{\mathcal{A}}\left(\mathcal{R}^{\left(n_{k}\right)}\right)=D O M^{\mathcal{A}}\left(\widetilde{\mathcal{R}_{k}}\right)$ and

$$
\left(\mathcal{R}^{\left(n_{k}\right)}\right)^{\mathcal{A}}(\underline{\mathfrak{A}})=\widetilde{\mathcal{R}}^{\mathcal{A}}(\underline{\mathfrak{A}}), \forall \underline{\mathfrak{A}} \in D O M^{\mathcal{A}}\left(\mathcal{R}^{\left(n_{k}\right)}\right),
$$

for $k=1,2$. Therefore $\widetilde{\mathcal{R}_{1}}$ and $\widetilde{\mathcal{R}_{2}}$ are minimal nc Fornasini-Marchesini realizations, both centred at $\underline{\tilde{Y}}$, of $R_{1}$ and $R_{2}$ - which are $\left(\mathbb{K}^{d}\right)_{n c}$-evaluation equivalent- hence Theorem 2.13 implies

$$
D O M_{p \ell m}\left(\widetilde{\mathcal{R}_{1}}\right)=D O M_{p \ell m}\left(\widetilde{\mathcal{R}_{2}}\right) \text { and } \widetilde{\mathcal{R}_{1}}(\underline{X})=\widetilde{\mathcal{R}_{2}}(\underline{X}), \forall \underline{X} \in D O M_{p \ell m}\left(\widetilde{\mathcal{R}_{1}}\right)
$$

for every $m \in \mathbb{N}$ and

$$
D O M^{\mathcal{A}}\left(\widetilde{\mathcal{R}_{1}}\right)=D O M^{\mathcal{A}}\left(\widetilde{\mathcal{R}_{2}}\right) \text { and }{\widetilde{\mathcal{R}_{1}}}^{\mathcal{A}}(\underline{\mathfrak{A}})={\widetilde{\mathcal{R}_{2}}}^{\mathcal{A}}(\underline{\mathfrak{A}}), \forall \underline{\mathfrak{A}} \in D O M^{\mathcal{A}}\left(\widetilde{\mathcal{R}_{1}}\right),
$$

which yield that

$$
D O M_{p \ell m}\left(\mathcal{R}_{1}\right)=D O M_{p \ell m}\left(\widetilde{\mathcal{R}_{1}}\right)=D O M_{p \ell m}\left(\widetilde{\mathcal{R}_{2}}\right)=D O M_{p \ell m}\left(\mathcal{R}_{2}\right)
$$

for every $m \in \mathbb{N}$ and

$$
\mathcal{R}_{1}(\underline{X})=\widetilde{\mathcal{R}_{1}}(\underline{X})=\widetilde{\mathcal{R}_{2}}(\underline{X})=\mathcal{R}_{2}(\underline{X}), \forall \underline{X} \in D O M_{p \ell m}\left(\mathcal{R}_{1}\right) .
$$

It is easily seen that $\underline{X} \in D O M_{p m}\left(\mathcal{R}_{k}\right) \Longleftrightarrow I_{\ell} \otimes \underline{X} \in D O M_{p \ell m}\left(\mathcal{R}_{k}\right)$ and in that case $\mathcal{R}_{k}\left(I_{\ell} \otimes \underline{X}\right)=I_{\ell} \otimes \mathcal{R}_{k}(\underline{X})$, where $k=1$ or $k=2$ and thus, from (3.2) and (3.3) one can get (3.1).

- Moreover,

$$
D O M^{\mathcal{A}}\left(\mathcal{R}^{\left(n_{1}\right)}\right)=D O M^{\mathcal{A}}\left(\widetilde{\mathcal{R}_{1}}\right)=D O M^{\mathcal{A}}\left(\widetilde{\mathcal{R}_{2}}\right)=D O M^{\mathcal{A}}\left(\mathcal{R}^{\left(n_{2}\right)}\right)
$$

implies that for every $\underline{\mathfrak{a}} \in \mathcal{A}^{d}$,

$$
\begin{aligned}
I_{s_{1}} \otimes \underline{\mathfrak{a}} \in D O M^{\mathcal{A}}\left(\mathcal{R}_{1}\right) & \Longleftrightarrow I_{n_{1}} \otimes\left(I_{s_{1}} \otimes \underline{\mathfrak{a}}\right) \in D O M^{\mathcal{A}}\left(\mathcal{R}^{\left(n_{1}\right)}\right) \\
I_{n_{2}} \otimes\left(I_{s_{2}} \otimes \underline{\mathfrak{a}}\right) \in D O M^{\mathcal{A}}\left(\mathcal{R}^{\left(n_{2}\right)}\right) & \Longleftrightarrow I_{s_{2}} \otimes \underline{\mathfrak{a}} \in D O M^{\mathcal{A}}\left(\mathcal{R}_{2}\right)
\end{aligned}
$$

and for every such $\underline{\mathfrak{a}}$ :

$$
\begin{aligned}
I_{n_{1}} \otimes \mathcal{R}_{1}^{\mathcal{A}}\left(I_{s_{1}} \otimes \underline{\mathfrak{a}}\right)= & \left(\mathcal{R}^{\left(n_{1}\right)}\right)^{\mathcal{A}}\left(I_{p \ell} \otimes \underline{\mathfrak{a}}\right)=\widetilde{\mathcal{R}}^{\mathcal{A}}\left(I_{p \ell} \otimes \underline{\mathfrak{a}}\right) \\
& =\widetilde{\mathcal{R}}^{\mathcal{A}}\left(I_{p \ell} \otimes \underline{\mathfrak{a}}\right)=\left(\mathcal{R}^{\left(n_{2}\right)}\right)^{\mathcal{A}}\left(I_{p \ell} \otimes \underline{\mathfrak{a}}\right)=I_{n_{2}} \otimes \mathcal{R}_{2}^{\mathcal{A}}\left(I_{s_{2}} \otimes \underline{\mathfrak{a}}\right),
\end{aligned}
$$

which then, as $s_{1} n_{1}=s_{2} n_{2}$, implies $I_{s_{2}} \otimes \mathcal{R}_{1}^{\mathcal{A}}\left(I_{s_{1}} \otimes \underline{\mathfrak{a}}\right)=I_{s_{1}} \otimes \mathcal{R}_{2}^{\mathcal{A}}\left(I_{s_{2}} \otimes \underline{\mathfrak{a}}\right)$.

Remark 3.2. If $R_{1}$ and $R_{2}$ are $\left(\mathbb{K}^{d}\right)_{n c}$-evaluation equivalent nc (non-degenerate) rational expressions in $x_{1}, \ldots, x_{d}$ over $\mathbb{K}$, as explained in the beginning of the proof, there exists $\underline{\tilde{Y}} \in \operatorname{dom}_{\ell p}\left(R_{1}\right) \cap \operatorname{dom}_{\ell p}\left(R_{2}\right)$ for some $\ell \in \mathbb{N}$, thus Theorem 2.13 implies that $L_{R_{1}}(\ell p)=L_{R_{2}}(\ell p)$ and hence

$$
\mathfrak{m}\left(R_{1}\right)=\frac{L_{R_{1}}(\ell p)}{\ell p}=\frac{L_{R_{2}}(\ell p)}{\ell p}=\mathfrak{m}\left(R_{2}\right) .
$$

Therefore, we define the McMillan degree of a nc rational function $\Re$ to be $\mathfrak{m}(\mathfrak{R}):=\mathfrak{m}(R)$ for every $R \in \mathfrak{R}$.

Recall that a nc rational function $\mathfrak{R}$ is an equivalence class of the form

$$
\mathfrak{R}=\{R: R \text { is a non-degenerate representative of } \mathfrak{R}\}
$$


whose elements are $\left(\mathbb{K}^{d}\right)_{n c}$-evaluation equivalent nc rational expressions in $x_{1}, \ldots, x_{d}$ over $\mathbb{K}$, whereas the domain and $\mathcal{A}$-domain of regularity of $\mathfrak{R}$ are given by

$$
\operatorname{dom}(\mathfrak{R})=\bigcup_{R \in \mathfrak{R}} \operatorname{dom}(R) \text { and } \operatorname{dom}^{\mathcal{A}}(\mathfrak{R})=\bigcup_{R \in \mathfrak{R}} \operatorname{dom}^{\mathcal{A}}(R),
$$

respectively. We now use Corollary 2.18 and Lemma 3.1, to show that the domain of regularity of a nc rational function $\mathfrak{R}$ at the level of $n \times n$ matrices, i.e.,

$$
\operatorname{dom}_{n}(\mathfrak{R})=\bigcup_{R \in \mathfrak{R}} \operatorname{dom}_{n}(R),
$$

lives inside the domain of any minimal nc Fornasini-Marchesini realization of a representative in $\mathfrak{R}$, up to a tensor product with the identity matrix.

Theorem 3.3. Let $\mathfrak{R} \in \mathbb{K} \nless x_{1}, \ldots, x_{d} \ngtr$ be a nc rational function. For every $n c$ rational expression $R \in \mathfrak{R}$, integer $s \in \mathbb{N}$, point $\underline{Y} \in \operatorname{dom}_{s}(\mathfrak{R})$, minimal nc FornasiniMarchesini realization $\mathcal{R}$ centred at $\underline{Y}$ of $R$, and unital stably finite $\mathbb{K}$-algebra $\mathcal{A}$, we have the following properties:

1. If $\underline{Z} \in \operatorname{dom}_{n}(\mathfrak{R})$, then $I_{s} \otimes \underline{Z} \in D O M_{s n}(\mathcal{R})$ and $I_{s} \otimes \mathfrak{R}(\underline{Z})=\mathcal{R}\left(I_{s} \otimes \underline{Z}\right)$.

2. If $s \mid n$, then $\operatorname{dom}_{n}(\mathfrak{R}) \subseteq D O M_{n}(\mathcal{R})$ and $\mathfrak{R}(\underline{Z})=\mathcal{R}(\underline{Z})$ for every $\underline{Z} \in$ $\operatorname{dom}_{n}(\Re)$.

3. If $\underline{\mathfrak{a}} \in \operatorname{dom}^{\mathcal{A}}(\mathfrak{R})$, then $I_{s} \otimes \underline{\mathfrak{a}} \in D O M^{\mathcal{A}}(\mathcal{R})$ and $I_{s} \otimes \mathfrak{R}^{\mathcal{A}}(\underline{\mathfrak{a}})=\mathcal{R}^{\mathcal{A}}\left(I_{s} \otimes \underline{\mathfrak{a}}\right)$.

Proof. Let $\underline{Z} \in \operatorname{dom}_{n}(\mathfrak{R})$, so there exists a nc rational expression $\widetilde{R} \in \mathfrak{R}$ such that $\underline{Z} \in \operatorname{dom}_{n}(\widetilde{R})$, while Corollary 2.18 implies the existence of a minimal nc FornasiniMarchesini realization $\widetilde{\mathcal{R}}$ of $\widetilde{R}$, centred at $\underline{Z}$. Then $\mathcal{R}$ and $\widetilde{\mathcal{R}}$ are minimal nc Fornasini-Marchesini realizations of $R$ and $\widetilde{R}$, with centres in $\left(\mathbb{K}^{s \times s}\right)^{d}$ and $\left(\mathbb{K}^{n \times n}\right)^{d}$, respectively. Since $R, \widetilde{R} \in \mathfrak{R}$, it follows that $R$ and $\widetilde{R}$ are $\left(\mathbb{K}^{d}\right)_{n c}$-evaluation equivalent, therefore Lemma 3.1 guarantees that

$$
D O M_{p m}(\widetilde{\mathcal{R}})=D O M_{p m}(\mathcal{R}) \text { and } \widetilde{\mathcal{R}}(\underline{X})=\mathcal{R}(\underline{X}), \forall \underline{X} \in D O M_{p m}(\mathcal{R})
$$

for every $m \in \mathbb{N}$, where $p=$ l.c. $m(s, n)$. Thus, $p \mid$ sn implies that

$$
\underline{Z} \in \operatorname{dom}_{n}(\widetilde{R}) \Longrightarrow I_{s} \otimes \underline{Z} \in \operatorname{dom}_{s n}(\widetilde{R}) \subseteq D O M_{s n}(\widetilde{\mathcal{R}})=D O M_{s n}(\mathcal{R})
$$

and hence

$$
I_{s} \otimes \mathfrak{R}(\underline{Z})=I_{s} \otimes \widetilde{R}(\underline{Z})=\widetilde{R}\left(I_{s} \otimes \underline{Z}\right)=\widetilde{\mathcal{R}}\left(I_{s} \otimes \underline{Z}\right)=\mathcal{R}\left(I_{s} \otimes \underline{Z}\right),
$$

which ends the proof of part 1.

- Suppose next that $s \mid n$; in that case we have $p=$ l.c.m $(s, n)=n$. Thus, in view of (3.4) with $m=1$, if $\underline{Z} \in \operatorname{dom}_{n}(\widetilde{R})$, then

$$
\underline{Z} \in D O M_{n}(\widetilde{\mathcal{R}})=D O M_{n}(\mathcal{R}) \text { and } \mathfrak{R}(\underline{Z})=\widetilde{R}(\underline{Z})=\widetilde{\mathcal{R}}(\underline{Z})=\mathcal{R}(\underline{Z}),
$$

which ends the proof of part 2.

- Finally, let $\mathfrak{a} \in \operatorname{dom}^{\mathcal{A}}(\mathfrak{R})$, then there exists a non-degenerate nc rational expression $\widehat{R} \in \mathfrak{R}$ such that $\underline{\mathfrak{a}} \in \operatorname{dom}^{\mathcal{A}}(\widehat{R})$. As $\widehat{R}$ is non-degenerate, there exists $t \in \mathbb{N}$ such that $\operatorname{dom}_{t}(\widehat{R}) \neq \emptyset$. Let $\underline{\underline{Y}} \in \operatorname{dom}_{t}(\widehat{R})$ and apply Corollary 2.18: so there exists a minimal realization $\widehat{\mathcal{R}}$ of $\widehat{R}$, centred at $\underline{\hat{Y}}$, such that

$$
\underline{\mathfrak{a}} \in \operatorname{dom}^{\mathcal{A}}(\widehat{R}) \Longrightarrow I_{t} \otimes \underline{\mathfrak{a}} \in D O M^{\mathcal{A}}(\widehat{\mathcal{R}}) \text { and } \widehat{\mathcal{R}}^{\mathcal{A}}\left(I_{t} \otimes \underline{\mathfrak{a}}\right)=I_{t} \otimes \widehat{R}^{\mathcal{A}}(\underline{\mathfrak{a}}) .
$$


As $\mathcal{R}$ and $\widehat{\mathcal{R}}$ are minimal nc Fornasini-Marchesini realizations of $R \in \mathfrak{R}$ and $\widehat{R} \in \mathfrak{R}$, respectively, Lemma 3.1 guarantees that

$$
I_{t} \otimes \underline{\mathfrak{a}} \in D O M^{\mathcal{A}}(\widehat{\mathcal{R}}) \Longrightarrow I_{s} \otimes \underline{\mathfrak{a}} \in D O M^{\mathcal{A}}(\mathcal{R})
$$

and also that

$$
I_{t} \otimes \mathcal{R}^{\mathcal{A}}\left(I_{s} \otimes \underline{\mathfrak{a}}\right)=I_{s} \otimes \widehat{\mathcal{R}}^{\mathcal{A}}\left(I_{t} \otimes \underline{\mathfrak{a}}\right)=I_{s} \otimes\left(I_{t} \otimes \widehat{R}^{\mathcal{A}}(\underline{\mathfrak{a}})\right),
$$

thus $\mathcal{R}^{\mathcal{A}}\left(I_{s} \otimes \underline{\mathfrak{a}}\right)=I_{s} \otimes \widehat{R}^{\mathcal{A}}(\underline{\mathfrak{a}})=I_{s} \otimes \mathfrak{R}^{\mathcal{A}}(\underline{\mathfrak{a}})$.

What we proved is that

$$
\operatorname{dom}_{s m}(\Re) \subseteq D O M_{s m}(\mathcal{R}), \forall m \in \mathbb{N}
$$

however in the case where $s=1$ and $\underline{Y}=(0, \ldots, 0)$, the nc Fornasini-Marchesini realization $\mathcal{R}$ is actually a $1 \times 1$ matrix valued nc rational expression (not a priori possible if $s>1$ ); by viewing $\mathfrak{R}$ as a $1 \times 1$ matrix valued nc rational function (cf. Remark 4.1), it follows that the nc Fornasini-Marchesini realization $\mathcal{R}$ is a representative of $\mathfrak{R}$ and therefore

$$
\operatorname{dom}_{m}(\mathfrak{R}) \supseteq D O M_{m}(\mathcal{R}), \forall m \in \mathbb{N} .
$$

In other words, by applying Theorem 3.3 we actually obtain a proof for Theorem 1 from the introduction which - unlike the original proof in [49] — does not make any use of the difference-differential calculus of nc functions.

Corollary 3.4. If $\mathfrak{R}$ is a $n$ c rational function of $x_{1}, \ldots, x_{d}$ over $\mathbb{K}$ and $\mathfrak{R}$ is regular at $\underline{0}$, then $\mathfrak{R}$ admits a unique (up to unique similarity) minimal (observable and controllable) nc Fornasini-Marchesini realization

$$
\mathfrak{R}\left(x_{1}, \ldots, x_{d}\right)=D+C\left(I_{L}-\sum_{k=1}^{d} A_{k} x_{k}\right)^{-1} \sum_{k=1}^{d} B_{k} x_{k},
$$

where $A_{1}, \ldots, A_{d} \in \mathbb{K}^{L \times L}, B_{1}, \ldots, B_{d} \in \mathbb{K}^{L \times 1}, C \in \mathbb{K}^{1 \times L}, D=\mathfrak{R}(\underline{0})$ and $L \in \mathbb{N}$,

$\operatorname{dom}_{m}(\Re)=\left\{\left(X_{1}, \ldots, X_{d}\right) \in\left(\mathbb{K}^{m \times m}\right)^{d}: \operatorname{det}\left(I_{L m}-X_{1} \otimes A_{1}-\ldots-X_{d} \otimes A_{d}\right) \neq 0\right\}$

for every $m \in \mathbb{N}$ and

$$
\mathfrak{R}\left(X_{1}, \ldots, X_{d}\right)=I_{m} \otimes D+\left(I_{m} \otimes C\right)\left(I_{m L}-\sum_{k=1}^{d} X_{k} \otimes A_{k}\right)^{-1} \sum_{k=1}^{d} X_{k} \otimes B_{k}
$$

for every $\left(X_{1}, \ldots, X_{d}\right) \in \operatorname{dom}_{m}(\Re)$.

\section{Realizations of Matrix Valued nC Rational Functions}

All of the analysis and results up to now can be generalized to the settings of matrix valued nc rational functions. In this section we describe the relevant definitions and main results in the matrix valued case.

If $\alpha, \beta \in \mathbb{N}$, we say that $\mathfrak{r}$ is a $\alpha \times \beta$ matrix valued nc rational function if $\mathfrak{r}$ is a $\alpha \times \beta$ matrix of nc rational functions, i.e., if

$$
\mathfrak{r}=\left[\mathfrak{R}_{i j}\right]_{1 \leq i \leq \alpha, 1 \leq j \leq \beta}
$$


where $\mathfrak{R}_{i j}$ are nc rational functions. The domain of regularity of $\mathfrak{r}$ is then defined by

$$
\operatorname{dom}(\mathfrak{r}):=\bigcap_{1 \leq i \leq \alpha, 1 \leq j \leq \beta} \operatorname{dom}\left(\Re_{i j}\right)
$$

and for every $\underline{X} \in \operatorname{dom}_{n}(\mathfrak{r})$ the evaluation $\mathfrak{r}(\underline{X})$ is given by

$$
\mathfrak{r}(\underline{X}):=E(n, \alpha)\left[\Re_{i j}(\underline{X})\right]_{1 \leq i \leq \alpha, 1 \leq j \leq \beta} E(n, \beta)^{T},
$$

where $E\left(\ell_{1}, \ell_{2}\right) \in \mathbb{K}^{\ell_{1} \ell_{2} \times \ell_{1} \ell_{2}}$ defined in (1.1). The need for the correction terms, which are shuffle matrices, is coming simply because otherwise evaluating $\mathfrak{r}$ term by term does not yield a nc function (it does not preserve direct sums), see e.g. [48, pp. 17-18]. If $\mathcal{A}$ is a unital $\mathbb{K}$-algebra, then the $\mathcal{A}$-domain of $\mathfrak{r}$ is defined by

$$
\operatorname{dom}^{\mathcal{A}}(\mathfrak{r}):=\bigcap_{1 \leq i \leq \alpha, 1 \leq j \leq \beta} \operatorname{dom}^{\mathcal{A}}\left(\Re_{i j}\right)
$$

and for every $\underline{\mathfrak{a}} \in \operatorname{dom}^{\mathcal{A}}(\mathfrak{r})$ the evaluation $\mathfrak{r}^{\mathcal{A}}(\underline{\mathfrak{a}})$ is given by

$$
\mathfrak{r}^{\mathcal{A}}(\underline{\mathfrak{a}}):=\left[\mathfrak{R}_{i j}^{\mathcal{A}}(\underline{\mathfrak{a}})\right]_{1 \leq i \leq \alpha, 1 \leq j \leq \beta} .
$$

Remark 4.1. In [49] the authors define matrix valued nc rational functions and their domains of regularity using equivalence classes of matrix valued nc rational expressions. However, it follows from [81, Lemma 3.9] that one can also define matrix valued nc rational functions as a matrix of nc rational functions, and the domains of regularity in both cases are equal.

Theorem 4.2. For every $\alpha \times \beta$ matrix valued nc rational function

$$
\mathfrak{r}=\left[\mathfrak{R}_{i j}\right]_{1 \leq i \leq \alpha, 1 \leq j \leq \beta} \text { and } \underline{Y}=\left(Y_{1}, \ldots, Y_{d}\right) \in \operatorname{dom}_{s}(\mathfrak{r}),
$$

there exist unique (up to unique similarity) $L \in \mathbb{N}, D \in \mathbb{K}^{\alpha s \times \beta s}, C \in \mathbb{K}^{\alpha s \times L}$, linear mappings $\mathbf{A}_{1}, \ldots, \mathbf{A}_{d}: \mathbb{K}^{s \times s} \rightarrow \mathbb{K}^{L \times L}$ and $\mathbf{B}_{1}, \ldots, \mathbf{B}_{d}: \mathbb{K}^{s \times s} \rightarrow \mathbb{K}^{L \times \beta s}$ such that $(\underline{\mathbf{A}}, \underline{\mathbf{B}})$ is controllable and $(C, \underline{\mathbf{A}})$ is observable, for which

$$
\operatorname{dom}_{s m}(\mathfrak{r}) \subseteq\left\{\underline{X} \in\left(\mathbb{K}^{s m \times s m}\right)^{d}: \operatorname{det}\left(I_{L m}-\sum_{k=1}^{d}\left(X_{k}-I_{m} \otimes Y_{k}\right) \mathbf{A}_{k}\right) \neq 0\right\}
$$

for every $m \in \mathbb{N}$ and $\mathfrak{r}(\underline{X})=\mathcal{R}(\underline{X})$ for every $\underline{X} \in \operatorname{dom}_{\text {sm }}(\mathfrak{r})$, where

$$
\mathcal{R}(\underline{X})=I_{m} \otimes D+\left(I_{m} \otimes C\right)\left(I_{L m}-\sum_{k=1}^{d}\left(X_{k}-I_{m} \otimes Y_{k}\right) \mathbf{A}_{k}\right)^{-1} \sum_{k=1}^{d}\left(X_{k}-I_{m} \otimes Y_{k}\right) \mathbf{B}_{k} .
$$

Moreover,

$$
\operatorname{dom}_{n}(\mathfrak{r}) \subseteq\left\{\underline{X} \in\left(\mathbb{K}^{n \times n}\right)^{d}: \operatorname{det}\left(I_{n L}-\sum_{k=1}^{d}\left(I_{s} \otimes X_{k}-I_{n} \otimes Y_{k}\right) \mathbf{A}_{k}\right) \neq 0\right\}
$$

for every $n \in \mathbb{N}$ and $I_{s} \otimes \mathfrak{r}(\underline{X})=\mathcal{R}\left(I_{s} \otimes \underline{X}\right)$ for every $\underline{X} \in \operatorname{dom}_{n}(\mathfrak{r})$. Furthermore, for any unital stably finite $\mathbb{K}$-algebra $\mathcal{A}$ :

$\operatorname{dom}^{\mathcal{A}}(\mathfrak{r}) \subseteq\left\{\underline{\mathfrak{a}} \in \mathcal{A}^{d}:\left(I_{L} \otimes 1_{\mathcal{A}}-\sum_{k=1}^{d}\left(I_{s} \otimes \mathfrak{a}_{k}-Y_{k} \otimes 1_{\mathcal{A}}\right) \mathbf{A}_{k}^{\mathcal{A}}\right)\right.$ is invertible in $\left.\mathcal{A}^{L \times L}\right\}$ 
and $I_{s} \otimes \mathfrak{r}^{\mathcal{A}}(\underline{\mathfrak{a}})=\mathcal{R}^{\mathcal{A}}\left(I_{s} \otimes \underline{\mathfrak{a}}\right)$ for every $\underline{\mathfrak{a}} \in \operatorname{dom}^{\mathcal{A}}(\mathfrak{r})$, i.e.,

$I_{s} \otimes \mathfrak{r}^{\mathcal{A}}(\underline{\mathfrak{a}})=D \otimes 1_{\mathcal{A}}+\left(C \otimes 1_{\mathcal{A}}\right)\left(I_{L} \otimes 1_{\mathcal{A}}-\sum_{k=1}^{d}\left(I_{s} \otimes \mathfrak{a}_{k}-Y_{k} \otimes 1_{\mathcal{A}}\right) \mathbf{A}_{k}^{\mathcal{A}}\right)^{-1} \sum_{k=1}^{d}\left(I_{s} \otimes \mathfrak{a}_{k}-Y_{k} \otimes 1_{\mathcal{A}}\right) \mathbf{B}_{k}^{\mathcal{A}}$

Similarity of nc Fornasini-Marchesini realizations of matrix valued nc rational functions is defined analogously to the case of (scalar) nc rational functions (cf. Theorem 2.13), as well as controllability and observability, which are defined via the controllability and un-observability subspaces of $\mathbb{K}^{L}$ (cf. Definition 2.7 and notice that the only difference is that the operators $\underline{\mathbf{A}}^{\omega} \cdot \mathbf{B}_{k}$ and $C \cdot \underline{\mathbf{A}}^{\omega}$ return matrices in $\mathbb{K}^{L \times \beta s}$ and $\mathbb{K}^{\alpha s \times L}$, respectively).

Proof. Suppose $\mathfrak{r}=\left[\Re_{i j}\right]_{1 \leq i \leq \alpha, 1 \leq j \leq \beta}$ is a $\alpha \times \beta$ matrix valued nc rational function and let $\underline{Y} \in \operatorname{dom}_{s}(\mathfrak{r})$. For every $1 \leq i \leq \alpha$ and $1 \leq j \leq \beta$ we have $\underline{Y} \in \operatorname{dom}_{s}\left(\mathfrak{R}_{i j}\right)$, while applying Theorem 3.3 , the nc rational function $\mathfrak{R}_{i j}$ admits a minimal nc Fornasini-Marchesini realization $\mathcal{R}_{i j}$ centred at $\underline{Y}$, described by a tuple $\left(L_{i j}, D^{i j}, C^{i j}, \underline{\mathbf{A}}^{i j}, \underline{\mathbf{B}}^{i j}\right)$, which is also a realization of $\Re_{i j}$ w.r.t $\mathcal{A}$.

- Let $\underline{X} \in \operatorname{dom}_{s m}(\mathfrak{r})$, then $\underline{X} \in \operatorname{dom}_{s m}\left(\Re_{i j}\right)$ and hence $\underline{X} \in D O M_{s m}\left(\mathcal{R}_{i j}\right)$ and $\mathfrak{R}_{i j}(\underline{X})=\mathcal{R}(\underline{X})$ for any $1 \leq i \leq \alpha$ and $1 \leq j \leq \beta$. Therefore

$$
\begin{gathered}
\mathfrak{r}(\underline{X})=E(s m, \alpha)\left[\begin{array}{ccc}
\mathfrak{R}_{11} & \ldots & \mathfrak{R}_{1 \beta} \\
\vdots & & \vdots \\
\mathfrak{R}_{\alpha 1} & \ldots & \mathfrak{R}_{\alpha \beta}
\end{array}\right](\underline{X}) E(s m, \beta)^{T}=E(s m, \alpha)\left[\begin{array}{ccc}
\mathcal{R}_{11} & \ldots & \mathcal{R}_{1 \beta} \\
\vdots & & \vdots \\
\mathcal{R}_{\alpha 1} & \ldots & \mathcal{R}_{\alpha \beta}
\end{array}\right](\underline{X}) \\
E(s m, \beta)^{T}=I_{m} \otimes D+\left(I_{m} \otimes C\right)\left(I_{L m}-\sum_{k=1}^{d}\left(X_{k}-I_{m} \otimes Y_{k}\right) \mathbf{A}_{k}\right)^{-1} \sum_{k=1}^{d}\left(X_{k}-I_{m} \otimes Y_{k}\right) \mathbf{B}_{k}=\mathcal{R}(\underline{X}),
\end{gathered}
$$

where the nc Fornasini-Marchesini realization $\mathcal{R}$ is described by

$$
\begin{aligned}
& L=\sum_{i=1}^{\alpha} \sum_{j=1}^{\beta} L_{i j}, D=E(s, \alpha)\left[\begin{array}{ccc}
D^{11} & \ldots & D^{1 \beta} \\
\vdots & & \vdots \\
D^{\alpha 1} & \ldots & D^{\alpha \beta}
\end{array}\right] E(s, \beta)^{T} \in \mathbb{K}^{\alpha s \times \beta s}, \\
& C=E(s, \alpha)\left[\begin{array}{ccccccccc}
C^{11} & \ldots & C^{1 \beta} & 0 & \ldots & \ldots & \ldots & \ldots & 0 \\
0 & \ldots & 0 & C^{21} & \ldots & C^{2 \beta} & 0 & \ldots & 0 \\
\vdots & & \vdots & \ddots & \ddots & \ddots & \vdots & & \vdots \\
0 & \ldots & \ldots & \ldots & \ldots & 0 & C^{\alpha 1} & \ldots & C^{\alpha \beta}
\end{array}\right] \in \mathbb{K}^{\alpha s \times L},
\end{aligned}
$$


with the linear mappings

$$
\mathbf{A}_{k}=\left[\begin{array}{ccccccc}
\mathbf{A}_{k}^{11} & \ldots & 0 & \ldots & \ldots & \ldots & 0 \\
\vdots & \ddots & & & & & \vdots \\
0 & & \mathbf{A}_{k}^{1 \beta} & & & & \vdots \\
\vdots & & & \ddots & & & \vdots \\
\vdots & & & & \mathbf{A}_{k}^{\alpha 1} & & 0 \\
\vdots & & & & & \ddots & \vdots \\
0 & \ldots & \ldots & \ldots & 0 & \ldots & \mathbf{A}_{k}^{\alpha \beta}
\end{array}\right], \mathbf{B}_{k}=\left[\begin{array}{ccc}
\mathbf{B}_{k}^{11} & \ldots & 0 \\
\vdots & \ddots & \vdots \\
0 & \ldots & \mathbf{B}_{k}^{1 \beta} \\
\mathbf{B}_{k}^{21} & \ldots & 0 \\
\vdots & \ddots & \vdots \\
0 & \ldots & \mathbf{B}_{k}^{2 \beta} \\
\vdots & \vdots & \vdots \\
\vdots & \vdots & \vdots \\
\mathbf{B}_{k}^{\alpha 1} & \ldots & 0 \\
\vdots & \ddots & \vdots \\
0 & \ldots & \mathbf{B}_{k}^{\alpha \beta}
\end{array}\right] E(s, \beta)^{T} .
$$

It is easily seen, by the diagonal structure of $\mathbf{A}_{k}$, that

$$
\operatorname{dom}_{s m}(\mathfrak{r}) \subseteq \bigcap_{1 \leq i \leq \alpha, 1 \leq j \leq \beta} \operatorname{DOM}_{s m}\left(\mathcal{R}_{i j}\right)=D O M_{s m}(\mathcal{R}) .
$$

- Moreover, if $\underline{X} \in \operatorname{dom}_{n}(\mathfrak{r})$, then $I_{s} \otimes \underline{X} \in \operatorname{dom}_{s n}(\mathfrak{r})$ and $\mathfrak{r}\left(I_{s} \otimes \underline{X}\right)=I_{s} \otimes \mathfrak{r}(\underline{X})$, while by the first part of the theorem we know that $I_{s} \otimes \underline{X} \in \operatorname{dom}_{s n}(\mathcal{R})$ and

$$
I_{s} \otimes \mathfrak{r}(\underline{X})=\mathfrak{r}\left(I_{s} \otimes \underline{X}\right)=\mathcal{R}\left(I_{s} \otimes \underline{X}\right) .
$$

- Furthermore, let $\underline{\mathfrak{a}} \in d o m^{\mathcal{A}}(\mathfrak{r})$, thus $\underline{\mathfrak{a}} \in \operatorname{dom}^{\mathcal{A}}\left(\mathfrak{R}_{i j}\right)$ and therefore $I_{s} \otimes \underline{\mathfrak{a}} \in$ $D O M^{\mathcal{A}}\left(\mathcal{R}_{i j}\right)$ and $\mathcal{R}_{i j}^{\mathcal{A}}\left(I_{s} \otimes \underline{\mathfrak{a}}\right)=I_{s} \otimes \mathfrak{R}_{i j}^{\mathcal{A}}(\underline{\mathfrak{a}})$, for every $1 \leq i \leq \alpha$ and $1 \leq j \leq \beta$. A direct and careful computation - which is omitted - shows that

$$
\begin{aligned}
\mathcal{R}^{\mathcal{A}}\left(I_{s}\right. & \otimes \underline{\mathfrak{a}})=\left(E(s, \alpha) \otimes 1_{\mathcal{A}}\right)\left[\mathcal{R}_{i j}^{\mathcal{A}}\left(I_{s} \otimes \underline{\mathfrak{a}}\right)\right]_{1 \leq i \leq \alpha, 1 \leq j \leq \beta}\left(E(s, \beta) \otimes 1_{\mathcal{A}}\right) \\
& =\left(E(s, \alpha) \otimes 1_{\mathcal{A}}\right)\left[I_{s} \otimes \mathfrak{R}_{i j}^{\mathcal{A}}(\underline{\mathfrak{a}})\right]_{1 \leq i \leq \alpha, 1 \leq j \leq \beta}\left(E(s, \beta) \otimes 1_{\mathcal{A}}\right)=I_{s} \otimes \mathfrak{r}^{\mathcal{A}}(\underline{\mathfrak{a}}) .
\end{aligned}
$$

- This proves the existence of a nc Fornasini-Marchesini realization for $\mathfrak{r}$, centred at $\underline{Y}$, that is also a realization of $\mathfrak{r}$ w.r.t $\mathcal{A}$. To obtain a minimal nc FornasiniMarchesini realization, we use the Kalman decomposition same as in Lemma 2.13, corresponding to the controllability and un-observability subspaces of $\mathbb{K}^{L}$, whereas the uniqueness (up to unique similarity) of such a minimal realization is proved with the same ingredients as in the proof of Theorem 2.13. The details of the proofs of the Kalman decomposition and the uniqueness are omitted.

Remark 4.3. It is not hard to see that the nc Fornasini-Marchesini realization built in the proof is not necessarily minimal, even if $\mathcal{R}_{i j}$ are all minimal nc FornasiniMarchesini realizations. However, the opposite is true, i.e., if $\mathcal{R}$ is a minimal nc Fornasini-Marchesini realization, then all of the nc Fornasini-Marchesini realizations $\mathcal{R}_{i j}$ must be minimal as well.

Remark 4.4. (cf. Remark 4.1) The proof of the existence part in Theorem 4.2 can be done using matrix valued nc rational expressions and the usual process of synthesis, yielding (4.2) for the a priori bigger domain of $\mathfrak{r}$, which uses matrix valued nc rational expressions and once again by [81, Lemma 3.9] is equal to the domain in the sense of (4.1). 
Remark 4.5 (McMillan degree of a matrix valued nc rational function). One can prove analogous versions of Theorem 2.21 and Remark 3.2, for matrix valued nc rational expressions and functions, thereby there exists an integer $\mathfrak{m}(\mathfrak{r})$ such that for any $\underline{Y} \in \operatorname{dom}_{s}(\mathfrak{r})$, we have

$$
L_{\mathfrak{r}}(\underline{Y})=s \cdot \mathfrak{m}(\mathfrak{r})
$$

here $L_{\mathfrak{r}}(\underline{Y})$ is the dimension of a minimal nc Fornasini-Marchesini realization of $\mathfrak{r}$, centred at $\underline{Y}$. We call $\mathfrak{m}(\mathfrak{r})$ the McMillan degree of $\mathfrak{r}$. It follows from Theorem 4.2 that

$$
\mathfrak{m}(\mathfrak{r})=\frac{L_{\mathfrak{r}}(\underline{Y})}{s} \leq \frac{\sum_{i=1}^{\alpha} \sum_{j=1}^{\beta} L_{i j}}{s}=\sum_{i=1}^{\alpha} \sum_{j=1}^{\beta} \mathfrak{m}\left(\Re_{i j}\right) .
$$

\section{Realizations of Hermitian nC Rational Functions}

In the case where $\mathbb{K}=\mathbb{R}$ or $\mathbb{K}=\mathbb{C}$, one often considers symmetric or hermitian nc rational expressions, specially with applications to free probability [16, 41, 75] and in optimization theory $[5,40,42,43]$. Unlike the case of descriptor realizations (see $[41,47]$ ), the expression for $\mathcal{R}_{\mathcal{F} \mathcal{M}}\left(\underline{X}^{*}\right)^{*}$ does not have the form of a nc Fornasini-Marchesini realization, for a nc Fornasini-Marchesini realization $\mathcal{R}_{\mathcal{F M}}$. Nevertheless, we can use our methods to obtain an analogue of Corollary 2.18 in the case where the function $\mathfrak{R}$ is hermitian, i.e., when

$$
\mathfrak{R}^{*}(\underline{X}):=\mathfrak{R}\left(\underline{X}^{*}\right)^{*}=\mathfrak{R}(\underline{X}) \text { for all } \underline{X} \in \operatorname{dom}(\mathfrak{R}),
$$

with the matrix pencil to be inverted having hermitian coefficients. We also get explicit (necessary and sufficient) conditions on the coefficients of the realization for the nc rational function to be hermitian.

Remark 5.1. One can define hermitian nc rational functions more precisely. First, one needs to define - using synthesis - a nc rational expression $R^{*}$, for any $n c$ rational expression $R$. Then, one can show that $R_{1}^{*} \sim R_{2}^{*}$, whenever $R_{1} \sim R_{2}$ (i.e., whenever $R_{1}$ and $R_{2}$ are $\left(\mathbb{K}^{d}\right)_{n c}$-evaluation equivalent). Finally, for every nc rational function $\mathfrak{R}$, let $\mathfrak{R}^{*}=\left\{R^{*}: R \in \mathfrak{R}\right\}$ and define $\mathfrak{R}$ to be hermitian if $\mathfrak{R}^{*}=\mathfrak{R}$, as equivalence classes.

We use the following notions: if $\mathbf{T}$ is a linear mapping on matrices, then $\mathbf{T}^{*}$ is the linear mapping given by $\mathbf{T}^{*}(X):=\mathbf{T}\left(X^{*}\right)^{*}$ and $\mathbf{T}$ is called hermitian if $\mathbf{T}^{*}=\mathbf{T}$. If $J$ is a square matrix of the form

$$
J=\left[\begin{array}{ccc}
I_{p} & 0 & 0 \\
0 & -I_{q} & 0 \\
0 & 0 & 0_{t}
\end{array}\right], \text { with } p, q, t \geq 0,
$$

then we say that $J$ is a semi-signature matrix; notice that if $t=0$, then $J$ is a signature matrix.

Theorem 5.2. Let $\mathfrak{R}$ be an hermitian nc rational function of $x_{1}, \ldots, x_{d}$ over $\mathbb{K}$, $\underline{Y}^{*}=\underline{Y} \in \operatorname{dom}_{s}(\mathfrak{R})$ and

$$
\mathcal{R}_{\mathcal{F M}}(\underline{X})=D+C\left(I_{L}-\sum_{k=1}^{d} \mathbf{A}_{k}\left(X_{k}-Y_{k}\right)\right)^{-1} \sum_{k=1}^{d} \mathbf{B}_{k}\left(X_{k}-Y_{k}\right)
$$

be a minimal nc Fornasini-Marchesini realization of $\mathfrak{R}$, centred at $\underline{Y}$. 
1. There exists a unique $S=S^{*} \in \mathbb{K}^{L \times L}$ such that

$D^{*}=D, \mathbf{A}_{k}^{*} \cdot S=S \cdot \mathbf{A}_{k}, \mathbf{B}_{k}^{*} \cdot S=C \cdot \mathbf{A}_{k}$ and $C \cdot \mathbf{B}_{k}=\left(C \cdot \mathbf{B}_{k}\right)^{*}, 1 \leq k \leq d$.

2. Once the relations in (5.1) hold, we have

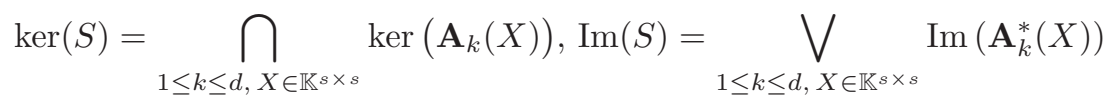

and

$$
\operatorname{ker}\left(C^{*}\right)=\bigcap_{1 \leq k \leq d, X \in \mathbb{K}^{s \times s}} \operatorname{ker}\left(\mathbf{B}_{k}(X)\right) .
$$

3. Symmetry of the minimal nc Fornasini-Marchesini realization: There exist $\check{C} \in \mathbb{K}^{s \times L}$, a semi-signature matrix $J \in \mathbb{K}^{L \times L}$ and hermitian linear mappings $\check{\mathbf{A}_{1}}, \ldots, \check{\mathbf{A}_{d}}: \mathbb{K}^{s \times s} \rightarrow \mathbb{K}^{L \times L}$, such that

$$
\mathcal{R}_{\mathcal{F M}}(\underline{X})=D+\check{C}\left(I_{L}-\sum_{k=1}^{d} \check{\mathbf{A}_{k}}\left(X_{k}-Y_{k}\right) J\right)^{-1} \sum_{k=1}^{d} \check{\mathbf{A}_{k}}\left(X_{k}-Y_{k}\right) \check{C}^{*},
$$

with $\left(\underline{\check{\mathbf{A}}} \cdot J, \underline{\check{\mathbf{A}}} \cdot \check{C}^{*}\right)$ controllable and $(\check{C}, \underline{\check{A}} \cdot J)$ observable. Conversely, if $\mathfrak{R}$ admits a realization of the form (5.3) with the controllability and observability conditions, then $\mathfrak{R}$ is an hermitian nc rational function.

4. Hermitian nc descriptor realization: There exist $D_{\mathcal{D}}=D_{\mathcal{D}}^{*} \in \mathbb{K}^{s \times s}, C_{\mathcal{D}} \in$ $\mathbb{K}^{s \times(L+s)}$, a signature matrix $J_{\mathcal{D}} \in \mathbb{K}^{(L+s) \times(L+s)}$ and hermitian linear mappings $\mathbf{A}_{1, \mathcal{D}}, \ldots, \mathbf{A}_{d, \mathcal{D}}: \mathbb{K}^{s \times s} \rightarrow \mathbb{K}^{(L+s) \times(L+s)}$, such that

$$
\mathcal{R}_{\mathcal{F M}}(\underline{X})=\mathcal{R}_{\mathcal{D}}(\underline{X}):=D_{\mathcal{D}}+C_{\mathcal{D}}\left(J_{\mathcal{D}}-\sum_{k=1}^{d} \mathbf{A}_{k, \mathcal{D}}\left(X_{k}-Y_{k}\right)\right)^{-1} C_{\mathcal{D}}^{*}
$$

and

$D O M_{s}\left(\mathcal{R}_{\mathcal{F M}}\right)=D O M_{s}\left(\mathcal{R}_{\mathcal{D}}\right):=\left\{\underline{X} \in\left(\mathbb{K}^{s \times s}\right)^{d}: \operatorname{det}\left(J_{\mathcal{D}}-\sum_{k=1}^{d} \mathbf{A}_{k, \mathcal{D}}\left(X_{k}-Y_{k}\right)\right) \neq 0\right\}$.

Moreover, similarly to Theorem 3.3, this also applies - after a suitable tensoring - to domains and evaluations on $n \times n$ matrices for all $n \in \mathbb{N}$ and w.r.t any unital stably finite $\mathbb{K}$-algebra $\mathcal{A}$.

5. The matrix $S$ is invertible $\Longleftrightarrow \bigcap_{1<k \leq d, X \in \mathbb{K}^{s \times s}} \operatorname{ker}\left(\mathbf{A}_{k}(X)\right)=\{\underline{0}\} \Longleftrightarrow$ there exists $Q \in \mathbb{K}^{L \times s}$ such that

$$
\mathbf{B}_{k}=\mathbf{A}_{k} \cdot Q, \forall 1 \leq k \leq d .
$$

In that case, $J \in \mathbb{K}^{L \times L}$ is invertible,

$$
\mathcal{R}_{\mathcal{F M}}(\underline{X})=D+\check{C} J\left(J-\sum_{k=1}^{d} \check{\mathbf{A}_{k}}\left(X_{k}-Y_{k}\right)\right)^{-1} \sum_{k=1}^{d} \check{\mathbf{A}_{k}}\left(X_{k}-Y_{k}\right) \check{C}{ }^{*},
$$

and

$$
\mathcal{R}_{\mathcal{F M}}(\underline{X})=\widetilde{D}+\check{C}\left(J-\sum_{k=1}^{d} \widetilde{\mathbf{A}_{k}}\left(X_{k}-Y_{k}\right)\right)^{-1} \check{C}^{*},
$$

where $\widetilde{\mathbf{A}_{k}}=J \check{\mathbf{A}_{k}} J$ and $\widetilde{D}=D-\check{C} J \check{C}^{*}$. 
Proof. 1. The proof of the first part of the theorem follows the same ideas as the proof of Theorem 2.13. As $\mathfrak{R}^{*}=\mathfrak{R}$ we obtain that $\mathcal{R}_{\mathcal{F} \mathcal{M}}^{*}=\mathcal{R}_{\mathcal{F M}}$ and then compare the coefficients in the Taylor-Taylor power series expansions

$$
\mathcal{R}_{\mathcal{F} \mathcal{M}}(\underline{X})=\sum_{\nu \in \mathcal{G}_{d}}\left(\underline{X}-I_{m} \otimes \underline{Y}\right)^{\odot_{s} \nu} \mathcal{R}_{\nu}
$$

and

$$
\mathcal{R}_{\mathcal{F} \mathcal{M}}(\underline{X})^{*}=\sum_{\nu \in \mathcal{G}_{d}}\left(\underline{X}-I_{m} \otimes \underline{Y}\right)^{\odot_{s} \nu} \mathcal{R}_{\nu, *},
$$

we obtain that $\mathcal{R}_{\nu}\left(Z_{1}, \ldots, Z_{\ell}\right)=\mathcal{R}_{\nu, *}\left(Z_{1}, \ldots, Z_{\ell}\right)$ for every $\nu=g_{i_{1}} \ldots g_{i_{\ell}} \in \mathcal{G}_{d}$ and $Z_{1}, \ldots, Z_{\ell} \in \mathbb{K}^{s \times s}$, where

$$
\mathcal{R}_{\nu}\left(Z_{1}, \ldots, Z_{\ell}\right)= \begin{cases}D & : \text { if } \ell=0 \\ C \mathbf{B}_{i_{1}}\left(Z_{1}\right) & : \text { if } \ell=1 \\ C \mathbf{A}_{i_{1}}\left(Z_{1}\right) \cdots \mathbf{A}_{i_{\ell-1}}\left(Z_{\ell-1}\right) \mathbf{B}_{i_{\ell}}\left(Z_{\ell}\right) & : \text { if } \ell>1\end{cases}
$$

and

$$
\mathcal{R}_{\nu, *}\left(Z_{1}, \ldots, Z_{\ell}\right)= \begin{cases}D^{*} & : \text { if } \ell=0 \\ \mathbf{B}_{i_{1}}^{*}\left(Z_{1}\right) C^{*} & : \text { if } \ell=1 \\ \mathbf{B}_{i_{1}}^{*}\left(Z_{1}\right) \mathbf{A}_{i_{2}}^{*}\left(Z_{2}\right) \cdots \mathbf{A}_{i_{\ell}}^{*}\left(Z_{\ell}\right) C^{*} & : \text { if } \ell>1 .\end{cases}
$$

For $\nu=\emptyset$ we get that $D=D^{*}$. Next, define a linear mapping

$$
\mathcal{S}\left(\underline{\mathbf{A}}^{\omega}\left(W_{1}, \ldots, W_{k}\right) \mathbf{B}_{j}(Z) \underline{u}\right)=\left(\underline{\mathbf{A}}^{*}\right)^{\omega}\left(W_{1}, \ldots, W_{k}\right) \mathbf{A}_{j}^{*}(Z) C^{*} \underline{u}
$$

for every $\omega \in \mathcal{G}_{d}$ of length $k \geq 0,1 \leq j \leq d, W_{1}, \ldots, W_{k}, Z_{j} \in \mathbb{K}^{s \times s}$ and $\underline{u} \in \mathbb{K}^{s}$, then we extend $\mathcal{S}$ by linearity.

- It is easily seen, from the controllability of $(\underline{\mathbf{A}}, \underline{\mathbf{B}})$ that $\mathcal{S}: \mathbb{K}^{L} \rightarrow \mathbb{K}^{L}$ is well defined: suppose

$$
\underline{w}_{1}=\sum_{l \in I_{L}} \underline{\mathbf{A}}^{\omega_{l}}(\underline{W}) \mathbf{B}_{j_{l}}\left(Z_{l}\right) \underline{u}_{l}=\sum_{t \in I_{T}} \underline{\mathbf{A}}^{\eta_{t}}(\underline{Q}) \mathbf{B}_{i_{t}}\left(P_{t}\right) \underline{v}_{t}=\underline{w}_{2},
$$

for $\omega_{l}, \eta_{t} \in \mathcal{G}_{d}, \underline{W}=\left(W_{1}^{(l)}, \ldots, W_{k_{l}}^{(l)}\right) \in\left(\mathbb{K}^{s \times s}\right)^{k_{l}}, \underline{Q}=\left(Q_{1}^{(t)}, \ldots, Q_{m_{t}}^{(t)}\right) \in\left(\mathbb{K}^{s \times s}\right)^{m_{t}}, Z_{l}, P_{t} \in$ $\mathbb{K}^{s \times s}, 1 \leq j_{l}, i_{t} \leq d$ and $\underline{u}_{l}, \underline{v}_{t} \in \mathbb{K}^{s}$ for every $l \in I_{L}$ and $t \in I_{T}$. Thus for every $1 \leq n \leq d, \alpha \in \mathcal{G}_{d}, \widetilde{X} \in \mathbb{K}^{s \times s}$ and $\underline{X}=\left(X_{1}, \ldots, X_{|\alpha|}\right) \in\left(\mathbb{K}^{s \times s}\right)^{|\alpha|}$ :

$$
\begin{aligned}
& \sum_{l \in I_{L}} \mathcal{R}_{g_{n} \alpha \omega_{l} g_{j_{l}}, *}\left(\tilde{X}, \underline{X}, \underline{W}, Z_{l}\right) \underline{u}_{l}=\sum_{l \in I_{L}} \mathcal{R}_{g_{n} \alpha \omega_{l} g_{j_{l}}}\left(\tilde{X}, \underline{X}, \underline{W}, Z_{l}\right) \underline{u}_{l}=C \mathbf{A}_{n}(\widetilde{X}) \underline{\mathbf{A}}^{\alpha}(\underline{X}) \underline{w}_{1} \\
= & C \mathbf{A}_{n}(\tilde{\widetilde{X}}) \underline{\mathbf{A}}^{\alpha}(\underline{X}) \underline{w}_{2}=\sum_{t \in I_{T}} \mathcal{R}_{g_{n} \alpha \eta_{t} g_{i_{t}}}\left(\widetilde{\widetilde{X}}, \underline{X}, \underline{Q}, P_{t}\right) \underline{v}_{t}=\sum_{t \in I_{T}} \mathcal{R}_{g_{n} \alpha \eta_{t} g_{i_{t}}, *}\left(\tilde{\widetilde{X}}, \underline{X}, \underline{Q}, P_{t}\right) \underline{v}_{t},
\end{aligned}
$$

which implies that

$$
\mathbf{B}_{n}^{*}(\tilde{X})\left(\underline{\mathbf{A}}^{*}\right)^{\alpha}(\underline{X}) \sum_{l \in I_{L}}\left(\underline{\mathbf{A}}^{*}\right)^{\omega_{l}}(\underline{W}) \mathbf{A}_{j_{l}}^{*}\left(Z_{l}\right) C^{*} \underline{u}_{l}=\mathbf{B}_{n}^{*}(\tilde{X})\left(\underline{\mathbf{A}}^{*}\right)^{\alpha}(\underline{X}) \sum_{t \in I_{T}}\left(\underline{\mathbf{A}}^{*}\right)^{\eta_{t}}(\underline{Q}) \mathbf{A}_{i_{t}}^{*}\left(P_{t}\right) C^{*} \underline{v}_{t},
$$

whereas the controllability of $(\underline{\mathbf{A}}, \underline{\mathbf{B}})$ implies that $\mathcal{S}\left(\underline{w}_{1}\right)=\mathcal{S}\left(\underline{w}_{2}\right)$.

- Let $S \in \mathbb{K}^{L \times L}$ be the matrix such that $\mathcal{S}(\underline{u})=S \underline{u}$ for every $\underline{u} \in \mathbb{K}^{L}$. We show that $S$ is self-adjoint: for every $\underline{u}, \underline{v} \in \mathbb{K}^{L}$ we use the controllability of $(\underline{\mathbf{A}}, \underline{\mathbf{B}})$ to write them as

$$
\underline{u}=\sum_{l \in I_{L}} \underline{\mathbf{A}}^{\omega_{l}}(\underline{W}) \mathbf{B}_{j_{l}}\left(Z_{l}\right) \underline{u}_{l} \text { and } \underline{v}=\sum_{t \in I_{T}} \underline{\mathbf{A}}^{\eta_{t}}(\underline{Q}) \mathbf{B}_{i_{t}}\left(P_{t}\right) \underline{v}_{t},
$$


thus, using the notation $\underline{Q}^{\#}:=\left(Q_{m_{t}}^{(t)}, \ldots, Q_{1}^{(t)}\right)$,

$$
\begin{aligned}
&\langle S \underline{u}, \underline{v}\rangle=\sum_{l \in I_{L}} \sum_{t \in I_{T}}\left\langle S \underline{\mathbf{A}}^{\omega_{l}}(\underline{W}) \mathbf{B}_{j_{l}}\left(Z_{l}\right) \underline{u}_{l}, \underline{\mathbf{A}}^{\eta_{t}}(\underline{Q}) \mathbf{B}_{i_{t}}\left(P_{t}\right) \underline{v}_{t}\right\rangle=\sum_{l \in I_{L}} \sum_{t \in I_{T}}\left\langle\left(\underline{\mathbf{A}}^{*}\right)^{\omega_{l}}(\underline{W}) \mathbf{A}_{j_{l}}^{*}\left(Z_{l}\right)\right. \\
&\left.C^{*} \underline{u}_{l}, \underline{\mathbf{A}}^{\eta_{t}}(\underline{Q}) \mathbf{B}_{i_{t}}\left(P_{t}\right) \underline{v}_{t}\right\rangle=\sum_{l \in I_{L}} \sum_{t \in I_{T}} \underline{v}_{t}^{*} \mathbf{B}_{i_{t}}^{*}\left(P_{t}\right)\left(\underline{\mathbf{A}}^{*}\right)^{\eta_{t}^{T}}\left(\underline{Q}^{\#}\right)\left(\underline{\mathbf{A}}^{*}\right)^{\omega_{l}}(\underline{W}) \mathbf{A}_{j_{l}}^{*}\left(Z_{l}\right) C^{*} \underline{u}_{l} \\
&= \sum_{l \in I_{L}} \sum_{t \in I_{T}} \underline{v}_{t}^{*} \mathcal{R}_{g_{i_{t}} \eta_{t}^{T} \omega_{l} g_{j_{l}}, *}\left(P_{t}, \underline{Q}^{\#}, \underline{W}, Z_{l}\right) \underline{u}_{l}=\sum_{l \in I_{L}} \sum_{t \in I_{T}} \underline{v}_{t}^{*} \mathcal{R}_{g_{i_{t}} \eta_{t}^{T} \omega_{l} g_{j_{l}}}\left(P_{t}, \underline{Q}^{\#}, \underline{W}, Z_{l}\right) \underline{u}_{l} \\
&= \sum_{l \in I_{L}} \sum_{t \in I_{T}} \underline{v}_{t}^{*} C \mathbf{A}_{i_{t}}\left(P_{t}\right) \underline{\mathbf{A}}^{\eta_{t}^{T}}\left(\underline{Q}^{\#}\right) \underline{\mathbf{A}}^{\omega_{l}}(\underline{W}) \mathbf{B}_{j_{l}}\left(Z_{l}\right) \underline{u}_{l}=\sum_{l \in I_{L}} \sum_{t \in I_{T}}\left\langle\underline{\mathbf{A}}^{\omega_{l}}(\underline{W}) \mathbf{B}_{j_{l}}\left(Z_{l}\right) \underline{u}_{l},\left(\underline{\mathbf{A}}^{*}\right)^{\eta_{t}}\right. \\
&\left.(\underline{Q}) \mathbf{A}_{i_{t}}^{*}\left(P_{t}\right) C^{*} \underline{v}_{t}\right\rangle=\sum_{l \in I_{L}} \sum_{t \in I_{T}}\left\langle\underline{\mathbf{A}}^{\omega_{l}}(\underline{W}) \mathbf{B}_{j_{l}}\left(Z_{l}\right) \underline{u}_{l}, S \underline{\mathbf{A}}^{\eta_{t}}(\underline{Q}) \mathbf{B}_{i_{t}}\left(P_{t}\right) \underline{v}_{t}\right\rangle=\langle\underline{u}, S \underline{v}\rangle,
\end{aligned}
$$

i.e., $S=S^{*}$.

- From (5.8) it follows that

$$
S \mathbf{B}_{k}(Z)=\mathbf{A}_{k}^{*}(Z) C^{*}, \forall 1 \leq k \leq d, Z \in \mathbb{K}^{s \times s}
$$

and for every $\underline{w}_{1}=\sum_{l \in I_{L}} \underline{\mathbf{A}}^{\omega_{l}}(\underline{W}) \mathbf{B}_{j_{l}}\left(Z_{l}\right) \underline{u}_{l} \in \mathbb{K}^{L}$ :

$$
\begin{gathered}
S \mathbf{A}_{k}(Z) \underline{w}_{1}=\sum_{l \in I_{L}} S \mathbf{A}_{k}(Z) \underline{\mathbf{A}}^{\omega_{l}}(\underline{W}) \mathbf{B}_{j_{l}}\left(Z_{l}\right) \underline{u}_{l}=\sum_{l \in I_{L}} \mathbf{A}_{k}^{*}(Z)\left(\underline{\mathbf{A}}^{*}\right)^{\omega_{l}}(\underline{W}) \mathbf{A}_{j_{l}}^{*}\left(Z_{l}\right) C^{*} \underline{u}_{l} \\
=\mathbf{A}_{k}^{*}(Z) \sum_{l \in I_{L}} S \underline{\mathbf{A}}^{\omega_{l}}(\underline{W}) \mathbf{B}_{j_{l}}\left(Z_{l}\right) \underline{u}_{l}=\mathbf{A}_{k}^{*}(Z) S \underline{w}_{1} .
\end{gathered}
$$

Once again, from the controllability of $(\underline{\mathbf{A}}, \underline{\mathbf{B}})$ we have

$$
S \mathbf{A}_{k}(Z)=\mathbf{A}_{k}^{*}(Z) S, \forall 1 \leq k \leq d, Z \in \mathbb{K}^{s \times s} .
$$

- It is easily seen that every self-adjoint matrix $S$ which satisfies the relations in (5.1), must satisfies (5.8) too. This implies the uniqueness of the matrix $S$.

2. Suppose all the relations in (5.1) hold.

- Let $\underline{v} \in \operatorname{ker}(S)$, thus (5.10) implies that $\underline{u}:=\mathbf{A}_{k}(Z) \underline{v} \in \operatorname{ker}(S)$ while (5.9) implies that $\underline{u} \in \operatorname{ker}(C)$. So $\underline{u} \in \operatorname{ker}(S)$ implies that $\mathbf{A}^{\omega}\left(X_{1}, \ldots, X_{|\omega|}\right) \underline{u} \in \operatorname{ker}(C)$, but $(C, \underline{\mathbf{A}})$ is observable, hence $\underline{u}=\underline{0}$ and $\underline{v} \in \operatorname{ker} \mathbf{A}_{k}(Z)$ for all $1 \leq k \leq d$ and $Z \in \mathbb{K}^{s \times s}$.

- On the other hand, if $\mathbf{A}_{k}(Z) \underline{v}=\underline{0}$ for all $1 \leq k \leq d$ and $Z \in \mathbb{K}^{s \times s}$, then $\mathbf{A}_{k}^{*}(Z) S \underline{v}=\underline{0}$ and also $\mathbf{B}_{k}(Z) S \underline{v}=\underline{0}$, whereas the controllability of $(\underline{\mathbf{A}}, \underline{\mathbf{B}})$ implies that $S \underline{v}=\underline{0}$.

- If $\mathbf{B}_{k}(Z) \underline{v}=\underline{0}$ for all $1 \leq k \leq d$ and $Z \in \mathbb{K}^{s \times s}$, then $\mathbf{A}_{k}^{*}(Z) C^{*} \underline{v}=\underline{0}$ and also $\mathbf{B}_{k}^{*}(Z) C^{*} \underline{v}=\underline{0}$, which implies that $C^{*} \underline{v}=\underline{0}$.

- On the other hand, if $\underline{v} \in \operatorname{ker}\left(C^{*}\right)$, then $\mathbf{B}_{k}(Z) \underline{v} \in \operatorname{ker}(C)$ and $S \mathbf{B}_{k}(Z) \underline{v}=\underline{0}$, i.e., $\mathbf{B}_{k}(Z) \underline{v} \in \operatorname{ker}(S)$. As $S$ is normal it implies that also $\mathbf{B}_{k}(Z) \underline{v} \in \operatorname{ker}(S)$ and thus $\mathbf{B}_{k}(Z) \underline{v} \in \operatorname{ker}\left(\mathbf{A}_{j}(\widetilde{Z})\right)$ for every $1 \leq j \leq d$ and $\widetilde{Z} \in \mathbb{K}^{s \times s}$, thus $\mathbf{B}_{k}(Z) \underline{v}=\underline{0}$.

3. The matrix $\left[\begin{array}{l}S \\ C\end{array}\right]$ is left invertible, as if $\underline{u} \in \operatorname{ker}\left[\begin{array}{l}S \\ C\end{array}\right]$, then $\underline{u} \in \operatorname{ker} C$ and

$$
\underline{u} \in \operatorname{ker}(S)=\bigcap_{1 \leq k \leq d, X \in \mathbb{K}^{s \times s}} \operatorname{ker}\left(\mathbf{A}_{k}(X)\right),
$$


which implies $\underline{u}=\underline{0}$, since $(C, \underline{\mathbf{A}})$ is observable.

- Let $K \in \mathbb{K}^{L \times(L+s)}$ be a left inverse of $\left[\begin{array}{l}S \\ C\end{array}\right]$ and $\widehat{\mathbf{A}_{k}}:=K \cdot\left[\begin{array}{l}\mathbf{A}_{k}^{*} \\ \mathbf{B}_{k}^{*}\end{array}\right]$, thus

$$
\mathbf{A}_{k}=K \cdot\left[\begin{array}{l}
S \\
C
\end{array}\right] \cdot \mathbf{A}_{k}=\widehat{\mathbf{A}_{k}} \cdot S, \mathbf{B}_{k}=K \cdot\left[\begin{array}{l}
S \\
C
\end{array}\right] \cdot \mathbf{B}_{k}=\widehat{\mathbf{A}_{k}} \cdot C^{*}
$$

and

$$
\begin{aligned}
& {\left[\begin{array}{l}
S \\
C
\end{array}\right] \cdot \widehat{\mathbf{A}_{k}} \cdot\left[\begin{array}{ll}
S & C^{*}
\end{array}\right]=\left[\begin{array}{ll}
S \cdot \widehat{\mathbf{A}_{k}} \cdot S & S \cdot \widehat{\mathbf{A}_{k}} \cdot C^{*} \\
C \cdot \widehat{\mathbf{A}_{k}} \cdot S & C \cdot \widehat{\mathbf{A}_{k}} \cdot C^{*}
\end{array}\right]=\left[\begin{array}{ll}
S \cdot \mathbf{A}_{k} & S \cdot \mathbf{B}_{k} \\
C \cdot \mathbf{A}_{k} & C \cdot \mathbf{B}_{k}
\end{array}\right] } \\
= & {\left[\begin{array}{ll}
\left(S \cdot \mathbf{A}_{k}\right)^{*} & \left(C \cdot \mathbf{A}_{k}\right)^{*} \\
\left(S \cdot \mathbf{B}_{k}\right)^{*} & \left(C \cdot \mathbf{B}_{k}\right)^{*}
\end{array}\right]=\left[\begin{array}{ll}
S \cdot \widehat{\mathbf{A}_{k}} \cdot S & S \cdot \widehat{\mathbf{A}}_{k}^{*} \cdot C^{*} \\
C \cdot \widehat{\mathbf{A}_{k}} \cdot S & C \cdot \widehat{\mathbf{A}_{k}} \cdot C^{*}
\end{array}\right]=\left[\begin{array}{l}
S \\
C
\end{array}\right] \cdot{\widehat{\mathbf{A}_{k}}}^{*} \cdot\left[\begin{array}{ll}
S & C^{*}
\end{array}\right], }
\end{aligned}
$$

which implies that $\widehat{\mathbf{A}_{k}}=\widehat{\mathbf{A}_{k}}$. Moreover, $\widehat{\mathbf{A}_{k}}$ is independent of the choice of the left inverse $K$ : if $K^{\prime}$ is another left inverse of $\left[\begin{array}{l}S \\ C\end{array}\right]$, then

$$
\left(K-K^{\prime}\right) \cdot\left[\begin{array}{l}
\mathbf{A}_{k}^{*} \\
\mathbf{B}_{k}^{*}
\end{array}\right] \cdot\left[\begin{array}{ll}
S & C^{*}
\end{array}\right]=\left[\begin{array}{ll}
\mathbf{A}_{k}-\mathbf{A}_{k} & \mathbf{B}_{k}-\mathbf{B}_{k}
\end{array}\right]=\left[\begin{array}{ll}
0 & 0
\end{array}\right]
$$

and the right invertibility of $\left[\begin{array}{ll}S & C^{*}\end{array}\right]$ implies that $K \cdot\left[\begin{array}{l}\mathbf{A}_{k}^{*} \\ \mathbf{B}_{k}^{*}\end{array}\right]=K^{\prime} \cdot\left[\begin{array}{l}\mathbf{A}_{k}^{*} \\ \mathbf{B}_{k}^{*}\end{array}\right]$. Notice that from the controllability of $(\underline{\mathbf{A}}, \underline{\mathbf{B}})$ we get

$$
\bigcap_{1 \leq k \leq d, X \in \mathbb{K}^{s \times s}} \operatorname{ker}\left(\widehat{\mathbf{A}_{k}}(X)\right)=\{\underline{0}\} .
$$

- Next, as $S=S^{*}$, one can write $S=T J T^{*}$, where $T \in \mathbb{K}^{L \times L}$ is invertible and $J \in \mathbb{K}^{L \times L}$ is a semi-signature matrix. Thus, using the relations in (5.11), we obtain

$$
\begin{aligned}
\mathcal{R}_{\mathcal{F M}}(\underline{X})=D+C & \left(I_{L}-\sum_{k=1}^{d} \widehat{\mathbf{A}_{k}}\left(X_{k}-Y_{k}\right) S\right)^{-1} \sum_{k=1}^{d} \widehat{\mathbf{A}_{k}}\left(X_{k}-Y_{k}\right) C^{*} \\
& =D+\check{C}\left(I_{L}-\sum_{k=1}^{d} \check{\mathbf{A}_{k}}\left(X_{k}-Y_{k}\right) J\right)^{-1} \sum_{k=1}^{d} \check{\mathbf{A}_{k}}\left(X_{k}-Y_{k}\right) \check{C}^{*},
\end{aligned}
$$

where $\check{C}=C\left(T^{*}\right)^{-1}$ and $\check{\mathbf{A}_{k}}=T^{*} \cdot \widehat{\mathbf{A}_{k}} \cdot T$ are hermitian, for $1 \leq k \leq d$. Moreover, it is easily seen that the controllability of $(\underline{\mathbf{A}}, \underline{\mathbf{B}})$ and the observability of $(C, \underline{\mathbf{A}})$ imply the controllability of $\left(\underline{\check{\mathbf{A}}} \cdot J, \underline{\check{\mathbf{A}}} \cdot \check{C}^{*}\right)$ and the observability of $(\check{C}, \underline{\check{\mathbf{A}}} \cdot J)$, respectively.

4. Define $E:=F+\check{C} J \check{C}^{*}$. The matrix

$$
\widetilde{S}=\left[\begin{array}{cc}
J & \check{C}^{*} \\
\check{C} & E
\end{array}\right] \in \mathbb{K}^{(L+s) \times(L+s)}
$$

is hermitian and invertible, whenever $F \succ 0$ or $F \prec 0$ : it is easily seen that $\widetilde{S}^{*}=\widetilde{S}$; let $\left[\begin{array}{l}\underline{u} \\ \underline{v}\end{array}\right] \in \operatorname{ker}(\widetilde{S})$, thus $J \underline{u}+\check{C}^{*} \underline{v}=\underline{0}$ and $\check{C} \underline{u}+E \underline{v}=\underline{0}$, therefore $\check{C}^{*} \underline{v}=-J \underline{u}$ and when we plug it in to the other equation we get $F \underline{v}=\check{C}\left(J^{2}-I_{L}\right) \underline{u}$. Thus, $-J \underline{u}=\check{C}^{*} F^{-1} \check{C}\left(J^{2}-I_{L}\right) \underline{u}$ and multiplying both sides by $\underline{u}^{*}\left(J^{2}-I_{L}\right)$ on the left, to get

$$
\underline{u}^{*}\left(J^{2}-I_{L}\right) \check{C}^{*} F^{-1} \check{C}\left(J^{2}-I_{L}\right) \underline{u}=-\underline{u}^{*}\left(J^{2}-I_{L}\right) J \underline{u}=-\underline{u}^{*}\left(J^{3}-J\right) \underline{u}=\underline{0} .
$$


Then $\underline{v}=\check{C}\left(J^{2}-I_{L}\right) \underline{u}=\underline{0}$, which implies that $J \underline{u}=\underline{0}$ and $\check{C} \underline{u}=\underline{0}$, i.e., that $\left[\begin{array}{l}J \\ \check{C}\end{array}\right] \underline{u}=\underline{0}$. Recall that $\left[\begin{array}{c}S \\ C\end{array}\right]=\left[\begin{array}{c}T J T^{*} \\ \check{C} T^{*}\end{array}\right]=\left[\begin{array}{cc}T & 0 \\ 0 & I_{s}\end{array}\right]\left[\begin{array}{c}J \\ \check{C}\end{array}\right] T^{*}$ is left invertible and hence $\left[\begin{array}{l}J \\ \check{C}\end{array}\right]$ is left invertible and hence $\underline{u}=\underline{0}$.

- As $\widetilde{S}$ is hermitian and invertible, there exist an invertible matrix $\widetilde{T} \in \mathbb{K}^{(L+s) \times(L+s)}$ and a signature matrix $J_{\mathcal{D}} \in \mathbb{K}^{(L+s) \times(L+s)}$ such that $\widetilde{S}^{-1}=\widetilde{T} J_{\mathcal{D}} \widetilde{T}^{*}$, therefore

$$
\begin{aligned}
& \mathcal{R}_{\mathcal{F M}}(\underline{X})=D-E+\left[\begin{array}{ll}
\check{C} & E
\end{array}\right]\left(I_{L+s}-\sum_{k=1}^{d}\left[\begin{array}{cc}
\check{\mathbf{A}_{k} J} & \check{\mathbf{A}_{k}} \check{C} \\
0 & 0
\end{array}\right]\left(X_{k}-Y_{k}\right)\right)^{-1}\left[\begin{array}{c}
0 \\
I_{s}
\end{array}\right]=D \\
& -E+\left[\begin{array}{ll}
0 & I_{s}
\end{array}\right] \widetilde{S}\left(I_{L+s}-\sum_{k=1}^{d}\left[\begin{array}{cc}
\check{\mathbf{A}_{k}} & 0 \\
0 & 0
\end{array}\right]\left(X_{k}-Y_{k}\right) \widetilde{S}\right)^{-1}\left[\begin{array}{c}
0 \\
I_{s}
\end{array}\right]=D-E+\left[\begin{array}{ll}
0 & I_{s}
\end{array}\right] \\
& \left(\widetilde{S}^{-1}-\sum_{k=1}^{d}\left[\begin{array}{cc}
\check{\mathbf{A}_{k}} & 0 \\
0 & 0
\end{array}\right]\left(X_{k}-Y_{k}\right)\right)^{-1}\left[\begin{array}{c}
0 \\
I_{s}
\end{array}\right]=D_{\mathcal{D}}+C_{\mathcal{D}}\left(J_{\mathcal{D}}-\sum_{k=1}^{d} \mathbf{A}_{k, \mathcal{D}}\left(X_{k}-Y_{k}\right)\right)^{-1} C_{\mathcal{D}}^{*}=\mathcal{R}_{\mathcal{D}}(\underline{X}),
\end{aligned}
$$

for every $\underline{X} \in D O M_{s}\left(\mathcal{R}_{\mathcal{F M}}\right)$, where

$$
D_{\mathcal{D}}:=D-F-\check{C} J \check{C}^{*}, C_{\mathcal{D}}:=\left[\begin{array}{cc}
0 & I_{s}
\end{array}\right] \widetilde{T}^{-*} \text { and } \mathbf{A}_{k, \mathcal{D}}:=\widetilde{T}^{-1}\left[\begin{array}{cc}
\check{\mathbf{A}}_{k} & 0 \\
0 & 0
\end{array}\right] \widetilde{T}^{-*},
$$

for every $1 \leq k \leq d$. We showed that $\mathcal{R}_{\mathcal{F} \mathcal{M}}(\underline{X})=\mathcal{R}_{\mathcal{D}}(\underline{X})$ and it is easily seen from the last computation that

$$
\underline{X} \in D O M_{s}\left(\mathcal{R}_{\mathcal{F M}}\right) \Longleftrightarrow \operatorname{det}\left(J_{\mathcal{D}}-\sum_{k=1}^{d} \mathbf{A}_{k, \mathcal{D}}\left(X_{k}-Y_{k}\right)\right) \neq 0,
$$

i.e., that $D O M_{s}\left(\mathcal{R}_{\mathcal{D}}\right)=D O M_{s}\left(\mathcal{R}_{\mathcal{F M}}\right)$.

- Furthermore, straight forward computations show that for every $m \geq 1$ :

$$
\underline{X} \in D O M_{s m}\left(\mathcal{R}_{\mathcal{F} \mathcal{M}}\right) \Longleftrightarrow \operatorname{det}\left(I_{m} \otimes J_{\mathcal{D}}-\sum_{k=1}^{d}\left(X_{k}-I_{m} \otimes Y_{k}\right) \mathbf{A}_{k, \mathcal{D}}\right) \neq 0,
$$

i.e., that $D O M_{s m}\left(\mathcal{R}_{\mathcal{F M}}\right)=D O M_{s m}\left(\mathcal{R}_{\mathcal{D}}\right)$ and also that $\mathcal{R}_{\mathcal{F M}}(\underline{X})=\mathcal{R}_{\mathcal{D}}(\underline{X})$, as well as that $D O M^{\mathcal{A}}\left(\mathcal{R}_{\mathcal{F} \mathcal{M}}\right)=D O M^{\mathcal{A}}\left(\mathcal{R}_{\mathcal{D}}\right)$ for any unital stably finite $\mathbb{K}$-algebra $\mathcal{A}$, i.e., for every $\underline{\mathfrak{A}} \in\left(\mathcal{A}^{s \times s}\right)^{d}$ :

$\underline{\mathfrak{A}} \in D O M^{\mathcal{A}}\left(\mathcal{R}_{\mathcal{F} \mathcal{M}}\right) \Longleftrightarrow\left(J_{\mathcal{D}} \otimes 1_{\mathcal{A}}-\sum_{k=1}^{d}\left(\mathfrak{A}_{k}-Y_{k} \otimes 1_{\mathcal{A}}\right) \mathbf{A}_{k, \mathcal{D}}^{\mathcal{A}}\right)$ is invertible in $\mathcal{A}^{(L+s) \times(L+s)}$

and for such $\underline{\mathfrak{A}}$ we have $\mathcal{R}_{\mathcal{F} \mathcal{M}}^{\mathcal{A}}(\underline{\mathfrak{A}})=\mathcal{R}_{\mathcal{D}}^{\mathcal{A}}(\underline{\mathfrak{A}})$.

5. If $S$ is invertible, then $\mathbf{B}_{k}^{*} \cdot S=C \cdot \mathbf{A}_{k}$ implies that $\mathbf{B}_{k}=S^{-1} \cdot \mathbf{A}_{k}^{*} \cdot C^{*}=\mathbf{A}_{k} \cdot S^{-1} C^{*}$ so we can choose $Q=S^{-1} C^{*}$ to get (5.5).

- On the other hand, suppose there exists $Q \in \mathbb{K}^{L \times s}$ such that $\mathbf{B}_{k}=\mathbf{A}_{k} \cdot Q$ for all $1 \leq k \leq d$. Thus, $C \cdot \mathbf{A}_{k}=\mathbf{B}_{k}^{*} \cdot S=Q^{*} \cdot \mathbf{A}_{k}^{*} \cdot S=Q^{*} S \cdot \mathbf{A}_{k}$ and also $C \cdot \mathbf{B}_{k}=C \cdot \mathbf{A}_{k} \cdot Q=Q^{*} S \cdot \mathbf{A}_{k} \cdot Q=Q^{*} S \cdot \mathbf{B}_{k}$, but the controllability of $(\underline{\mathbf{A}}, \underline{\mathbf{B}})$ implies that $C=Q^{*} S$. Therefore, $\operatorname{ker}(S) \subseteq \operatorname{ker}(C)$ but as

$$
\operatorname{ker}(S)=\bigcap_{1 \leq k \leq d, X \in \mathbb{K}^{s \times s}} \operatorname{ker}\left(\mathbf{A}_{k}(X)\right),
$$


it follows from the observability of $(C, \underline{\mathbf{A}})$ that $\operatorname{ker}(S)=\{\underline{0}\}$, i.e., that $S$ is invertible.

- If $S$ is invertible, then $J$ is invertible and the realization (5.6) is obtained from the realization (5.3) immediately.

- Finally, from (5.6) we get

$$
\begin{array}{r}
\mathcal{R}_{\mathcal{F M}}(\underline{X})=D+\check{C} J\left(J-\sum_{k=1}^{d} \check{\mathbf{A}_{k}}\left(X_{k}-Y_{k}\right)\right)^{-1}\left(\sum_{k=1}^{d} \check{\mathbf{A}_{k}}\left(X_{k}-Y_{k}\right)-J+J\right) \check{C}^{*} \\
=D-\check{C} J \check{C}^{*}+\check{C}\left(J-\sum_{k=1}^{d} \widetilde{\mathbf{A}_{k}}\left(X_{k}-Y_{k}\right)\right)^{-1} \check{C}^{*},
\end{array}
$$

that is the realization in $(5.7)$

Remark 5.3. If $\mathfrak{R}$ is an hermitian nc rational function, there exists $\underline{Y} \in \operatorname{dom}(\mathfrak{R})$ such that $\underline{Y}^{*}=\underline{Y}$; for a proof see [82, pp. 28-29].

Remark 5.4. We leave it to a future work, to describe connections between properties of the (semi-)signature matrices, $J$ and $J_{\mathcal{D}}$, appear in Theorem 5.2 and properties of the function $\mathfrak{R}$, such as perhaps (matrix) convexity (cf. [43]).

Remark 5.5. We say that a nc rational function $\mathfrak{R}$ admits a descriptor realization

$$
\mathcal{R}_{\mathcal{D}}(\underline{X})=C_{\mathcal{D}}\left(I_{L_{\mathcal{D}}}-\sum_{k=1}^{d} \mathbf{A}_{k, \mathcal{D}}\left(X_{k}-Y_{k}\right)\right)^{-1} B_{\mathcal{D}}
$$

centred at $\underline{Y} \in\left(\mathbb{K}^{s \times s}\right)^{d}$, if $\operatorname{dom}_{s m}(\mathfrak{R}) \subseteq D O M_{s m}\left(\mathcal{R}_{\mathcal{D}}\right)$ and $\mathfrak{R}(\underline{X})=\mathcal{R}_{\mathcal{D}}(\underline{X})$ for every $\underline{X} \in \operatorname{dom}_{s m}(\mathfrak{R})$, cf. Definition 2.3. We present some relations between Fornasini-Marchesini realizations and descriptor realizations (not necessarily in the symmetric case), without precise definitions of controllability and observability, as well as the McMillan degree (denoted by $\operatorname{Deg}_{s, \mathcal{D}}(\mathfrak{R})$ ), of descriptor realization:

- If $\Re$ admits a descriptor realization centred at $\underline{Y}$, described by $\left(L_{\mathcal{D}}, C_{\mathcal{D}}, \underline{\mathbf{A}}_{\mathcal{D}}, B_{\mathcal{D}}\right)$, then it admits a nc Fornasini-Marchesini realization described by

$L_{\mathcal{F M}}=L_{\mathcal{D}}, D_{\mathcal{F} \mathcal{M}}=C_{\mathcal{D}} B_{\mathcal{D}}, C_{\mathcal{F M}}=C_{\mathcal{D}}, \mathbf{A}_{k, \mathcal{F} \mathcal{M}}=\mathbf{A}_{k, \mathcal{D}}$ and $\mathbf{B}_{k, \mathcal{F} \mathcal{M}}=\mathbf{A}_{k, \mathcal{D}} B_{\mathcal{D}}$, for $1 \leq k \leq d, \mathcal{R}_{\mathcal{D}}$ is observable if and only if $\mathcal{R}_{\mathcal{F M}}$ is observable, and if $\mathcal{R}_{\mathcal{F M}}$ is controllable then $\mathcal{R}_{\mathcal{D}}$ is controllable. Therefore we have the relation

$$
\mathfrak{m}(\Re) s \leq \operatorname{Deg}_{s, \mathcal{D}}(\mathfrak{R}) .
$$

- If $\mathfrak{R}$ admits a nc Fornasini-Marchesini realization centred at $\underline{Y} \in\left(\mathbb{K}^{s \times s}\right)^{d}$, described by $\left(L_{\mathcal{F} \mathcal{M}}, D_{\mathcal{F} \mathcal{M}}, C_{\mathcal{F M}}, \underline{\mathbf{A}}_{\mathcal{F M}}, \underline{\mathbf{B}}_{\mathcal{F M}}\right)$, then $\mathfrak{R}$ admits a descriptor realization described by

$L_{\mathcal{D}}=L_{\mathcal{F} \mathcal{M}}+s, C_{\mathcal{D}}=\left[\begin{array}{ll}C_{\mathcal{F M}} & D_{\mathcal{F} \mathcal{M}}\end{array}\right], \mathbf{A}_{k, \mathcal{D}}=\left[\begin{array}{cc}\mathbf{A}_{k, \mathcal{F} \mathcal{M}} & \mathbf{B}_{k, \mathcal{F} \mathcal{M}} \\ 0 & 0_{s \times s}\end{array}\right]$ and $B_{\mathcal{D}}=\left[\begin{array}{c}0_{L_{\mathcal{F} \mathcal{M}} \times s} \\ I_{s}\end{array}\right]$,

for $1 \leq k \leq d, \mathcal{R}_{\mathcal{F M}}$ is controllable if and only if $\mathcal{R}_{\mathcal{D}}$ is controllable, and if $\mathcal{R}_{\mathcal{D}}$ is observable then $\mathcal{R}_{\mathcal{F M}}$ is observable. Therefore we have the relation

$$
D e g_{s, \mathcal{D}}(\mathfrak{R}) \leq(\mathfrak{m}(\Re)+1) s .
$$

- The inequalities (5.12) and (5.13) imply that

$$
\mathfrak{m}(\mathfrak{R}) s \leq \operatorname{Deg}_{s, \mathcal{D}}(\mathfrak{R}) \leq(\mathfrak{m}(\mathfrak{R})+1) s,
$$


whereas an analogue of Lemma 2.23 for descriptor realizations guarantees that $s \mid$ $D e g_{s, \mathcal{D}}(\mathfrak{R})$, then which then imply that

$$
\operatorname{Deg}_{s, \mathcal{D}}(\mathfrak{R})=\mathfrak{m}(\mathfrak{R}) s \text { or } \operatorname{Deg}_{s, \mathcal{D}}(\mathfrak{R})=(\mathfrak{m}(\mathfrak{R})+1) s .
$$

\section{REFERENCES}

[1] J. Agler and J. E. McCarthy. Global holomorphic functions in several non-commuting variables. Canadian Journal of Mathematics 67(2): pp. 241-285, 2015.

[2] J. Agler and J. E. McCarthy. Pick interpolation for free holomorphic functions. American Journal of Mathematics 137(6): pp. 1685-1701, 2015.

[3] J. Agler and J. E. McCarthy. Aspects of non-commutative function theory. Concrete Operators 3(1): pp. 15-24, 2016.

[4] D. Alpay and D. S. Kaliuzhnyi-Verbovetskyi. On the intersection of null spaces for matrix substitutions in a non-commutative rational formal power series. Comptes Rendus Mathematique 339(8): pp. 533-538, 2004.

[5] D. Alpay and D. S. Kaliuzhnyi-Verbovetzkyi. Matrix $J$-unitary noncommutative rational formal power series. The state space method generalizations and applications, vol. 161 of Operator Theory: Advances and Applications, Birkhäuser-Verlag, Basel, pp. 49-113, 2005.

[6] S. A. Amitsur. Rational identities and applications to algebra and geometry. Journal of Algebra 3(3): pp. 304-359, 1966.

[7] J. A. Ball and N. Cohen. De Branges-Rovnyak operator models and systems theory: a survey. Topics in Matrix and Operator Theory, vol. 50 of Operator Theory: Advances and Applications, Birkhäuser-Verlag, Boston, pp. 93-136, 1991.

[8] J. A. Ball, G. Groenewald and T. Malakorn. Structured noncommutative multidimensional linear systems. SIAM journal on control and optimization 44(4): pp. 1474-1528, 2005.

[9] J. A. Ball, G. Groenewald and T. Malakorn. Conservative structured noncommutative multidimensional linear systems. The state space method generalizations and applications, vol. 161 of Operator Theory: Advances and Applications, Birkhäuser, Basel, pp. 179-223, 2006.

[10] J. A. Ball, G. Groenewald, and T. Malakorn. Bounded real lemma for structured noncommutative multidimensional linear systems and robust control. Multidimensional Systems and Signal Processing 17(2-3): pp. 119-150, 2006.

[11] J. A. Ball and D. S. Kaliuzhnyi-Verbovetskyi. Conservative dilations of dissipative multidimensional systems: The commutative and non-commutative settings. Multidimensional Systems and Signal Processing 19(1): pp. 79-122, 2008.

[12] J. A. Ball and V. Vinnikov. Lax-Phillips scattering and conservative linear systems: a Cuntzalgebra multidimensional setting. Memoirs of the American Mathematical Society, 178(837), 2005.

[13] H. Bart, I. Gohberg and M. A. Kaashoek. Minimal factorization of matrix and operator functions. Operator Theory: Advances and Applications, Birkhäuser-Verlag, Basel, 1979.

[14] C. Beck. On formal power series representations for uncertain systems. IEEE Transactions on Automatic Control 46(2): pp. 314-319, 2001.

[15] C. L. Beck, J. Doyle, and K. Glover. Model reduction of multidimensional and uncertain systems. IEEE Transactions on Automatic Control 41(10): pp. 1466-1477, 1996.

[16] S. T. Belinschi, T. Mai and R. Speicher. Analytic subordination theory of operator-valued free additive convolution and the solution of a general random matrix problem. Journal für die reine und angewandte Mathematik (Crelles Journal) 732: pp. 21-53, 2017.

[17] G. M. Bergman. Skew fields of noncommutative rational functions, after Amitsur. Seminaire Schützenberger-Lentin-Nivat, Année 16, Paris, 1969/70

[18] J. Berstel and C. Reutenauer. Rational series and their languages. EATCS Monographs on Theoretical Computer Science 12, Springer-Verlag, Berlin, 1988.

[19] J. F. Camino, J. W. Helton, R. E. Skelton and J. Ye. Matrix inequalities: a symbolic procedure to determine convexity automatically. Integral Equations and Operator Theory 46(4): pp. 399-454, 2003.

[20] P. M. Cohn. On the embedding of rings in skew fields. Proceedings of the London Mathematical Society 3(1): pp. 511-530, 1961.

[21] P. M. Cohn. The embedding of firs in skew fields. Proceedings of the London Mathematical Society 3(2): pp. 193-213, 1971. 
[22] P. M. Cohn. Universal skew fields of fractions. Symposia Mathematica 8: pp. 135-148, 1972.

[23] P. M. Cohn. Free rings and their relations. London Mathematical Society Monographs 2. Academic Press, London, 1971.

[24] P. M. Cohn. The universal field of fractions of a semifir I. Numerators and denominators. Proceedings of the London Mathematical Society 3(1): pp. 1-32, 1982.

[25] P. M. Cohn. Skew fields. Theory of general division rings. Encyclopedia of Mathematics and its Applications 57, Cambridge University Press, Cambridge, 1995.

[26] P. M. Cohn. Free ideal rings and localization in general rings. New Mathematical Monographs 3, Cambridge University Press, Cambridge, 2006.

[27] P. M. Cohn and C. Reutenauer. A normal form in free fields. Canadian Journal of Mathematics 46(3): pp. 517-531, 1994.

[28] P. M. Cohn and C. Reutenauer. On the construction of the free field. International Journal of Algebra and Computation 9(3-4): pp. 307-323, 1999.

[29] E. G. Effros. Advances in quantized functional analysis. Proceedings of the International Congress of Mathematicians, Berkeley, pp. 906-916, 1986.

[30] M. Fliess. Sur le plongement de l'algèbre des séries rationnelles non commutatives dans un corps gauche. Comptes Rendus de l'Académie des Sciences Ser. A 271, pp. 926-927, 1970.

[31] M. Fliess. Matrices de Hankel. Journal de Mathématiques Pures et Appliquées 53(9): pp. 197-222, 1974

[32] M. Fliess. Sur divers produits de series formelles. Bulletin de la Société Mathématique de France 102: pp. 181-191, 1974.

[33] E. Fornasini and G. Marchesini. Doubly-indexed dynamical systems: state-space models and structural properties. Mathematical systems theory 12(1): pp. 59-72, 1978-1979.

[34] E. Fornasini and G. Marchesini. A critical review of recent results on 2-D system theory. Control Science and Technology for the Progress of Society 1 (Kyoto, 1981), IFAC, Laxenburg, pp. 255-261, 1982.

[35] K. Galkowski. Minimal state-space realization for a class of linear, discrete, nD, SISO systems. International Journal of Control 74(13): pp. 1279-1294, 2001.

[36] J. W. Helton. "Positive" noncommutative polynomials are sums of squares. Annals of Mathematics 156(2): pp. 675-694, 2002.

[37] J. W. Helton. Manipulating matrix inequalities automatically. Mathematical systems theory in biology, communications, computation, and finance, Springer, New York, pp. 237-256, 2003.

[38] J. W. Helton, I. Klep and S. McCullough. Analytic mappings between noncommutative pencil balls. Journal of Mathematical Analysis and Applications 376(2): pp. 407-428, 2011.

[39] J. W. Helton, I. Klep and S. McCullough. Proper Analytic Free Maps. Journal of Functional Analysis 260(5): pp. 1476-1490, 2011.

[40] J. W. Helton, I. Klep, S. McCullough and J. Volčič. Noncommutative polynomials describing convex sets. ArXiv preprint, https://arxiv.org/abs/1808.06669, 2018.

[41] J. W. Helton, T. Mai and R. Speicher. Applications of realizations (aka linearizations) to free probability. Journal of Functional Analysis 274(1): pp. 1-79, 2018.

[42] J. W. Helton and S. McCullough. Every convex free basic semi-algebraic set has an LMI representation. Annals of Mathematics 176(2): pp. 979-1013, 2012.

[43] J. W. Helton, S. McCullough and V. Vinnikov. Noncommutative convexity arises from linear matrix inequalities. Journal of Functional Analysis 240(1): pp. 105-191, 2006.

[44] R. A. Horn and C. R. Johnson. Topics in matrix analysis. Corrected reprint of the 1991 original, Cambridge University Press, 1994.

[45] I. Hughes. Division rings of fractions for group rings. Communications on Pure and Applied Mathematics 23(2): pp. 181-188, 1970.

[46] T. Kaczorek. Two dimensional linear systems. Lecture Notes in Control and Information Sciences 68, Springer-Verlag, Berlin, 1985.

[47] D. S. Kaliuzhnyi-Verbovetskyi and V. Vinnikov. Foundations of free noncommutative function theory. Mathematical Surveys and Monographs 199, American Mathematical Society, Providence, RI, 2014.

[48] D. S. Kaliuzhnyi-Verbovetskyi and V. Vinnikov. Noncommutative rational functions, their difference-differential calculus and realizations. Multidimensional Systems and Signal Processing 23(1-2): pp. 49-77, 2012. 
[49] D. S. Kaliuzhnyi-Verbovetskyi and V. Vinnikov. Singularities of rational functions and minimal factorizations: the noncommutative and the commutative settings. Linear Algebra and its Applications 430(4): pp. 869-889, 2009.

[50] R. E. Kalman, M. A. Arbib and P. L. Falb. Topics in mathematical systems theory. New York, NY, USA: McGraw Hill, 1969.

[51] S. C. Kleene. Representation of events in nerve nets and finite automata. Automata Studies, Annals of Mathematics Studies 34: pp. 3-41, Princeton University Press, Princeton, N.J., 1956.

[52] J. Lewin. Fields of fractions for group algebras of free groups. Transactions of the American Mathematical Society 192: pp. 339-346, 1974.

[53] A. I. Lichtman. On universal fields of fractions for free algebras. Journal of Algebra 231(2): pp. 652-676, 2000.

[54] P. A. Linnell. Division rings and group von Neumann algebras. Forum Mathematicum 5(5): pp. 561-576, 1993.

[55] P. A. Linnell. Noncommutative localization in group rings. Non-commutative localization in algebra and topology, vol. 330 of London Mathematical Society Lecture Note Series, pp. 40-59, Cambridg University Press, Cambridge, 2006.

[56] W. M. Lu, K. Zhou and J. C. Doyle. Stabilization of uncertain linear systems: an LFT approach. IEEE Transactions on Automatic Control 41(1): pp. 50-65, 1996.

[57] P. S. Muhly and B. Solel. Progress in noncommutative function theory. Science China Mathematics 54(11): pp. 2275-2294, 2011.

[58] A. Nemirovskii. Advances in convex optimization: conic programming. Plenary Lecture, International Congress of Mathematicians 1: pp. 413-444, Madrid, Spain, 2006.

[59] Y. Nesterov and A. Nemirovskii. Interior-point polynomial algorithms in convex programming. Studies in Applied Mathematics 13, Philadelphia, PA, 1994.

[60] V. Paulsen. Completely bounded maps and operator algebras. Cambridge Studies in Advanced Mathematics 78, Cambridge University Press, Cambridge, 2002.

[61] G. Popescu. Free holomorphic functions on the unit ball of $B(H)^{n}$. Journal of Functional Analysis 241(1): pp. 268-333, 2006.

[62] G. Popescu. Free holomorphic automorphisms of the unit ball of $B(H)^{n}$. Journal fr die reine und angewandte Mathematik (Crelles Journal) 638: pp. 119-168, 2010.

[63] M. Porat and V. Vinnikov. Realizations of non-commutative rational functions around a matrix centre, II: The lost-abbey conditions. to appear.

[64] M. Porat and V. Vinnikov. Realizations of non-commutative rational functions around a matrix centre, III: Functional models, Kronecker-Fliess theorem and the free skew field. to appear.

[65] C. Reutenauer. Malcev-Neumann series and the free field. Expositiones Mathematicae 17(5): pp. 469-478, 1999.

[66] L. H. Rowen. Polynomial identities in ring theory. Pure and Applied Mathematics 84, Academic Press, New York-London, 1980.

[67] R. E. Skelton, T. Iwasaki and K. M. Grigoriadis. A Unified Algebraic Approach to Linear Control Design. Taylor \& Francis, 1997.

[68] K. Schrempf. A standard form in (some) free fields: How to construct minimal linear representations. ArXiv preprint, https://arxiv.org/abs/1803.10627, 2018.

[69] K. Schrempf. Free fractions: An invitation to (applied) free fields. ArXiv preprint, https://arxiv.org/abs/1809.05425, 2018.

[70] K. Schrempf. Linearizing the word problem in (some) free fields. International Journal of Algebra and Computation 28(7): pp. 1209-1230, 2018.

[71] K. Schrempf. On the factorization of non-commutative polynomials (in free associative algebras). Journal of Symbolic Computation 94: pp. 126-148, 2019.

[72] M. P. Schützenberger. On the definition of a family of automata. Information and control 4(2-3): pp. 245-270, 1961.

[73] M. P. Schützenberger. Certain elementary families of automata. Proceedings of the Symposium on Mathematical Theory of Automata (New York, 1962), pp. 139-153, Polytechnic Press of Polytechnic Institute of Brooklyn, Brooklyn, New York, 1963.

[74] R. Speicher. Polynomials in asymptotically free random matrices. Acta Physica Polonica B 46(9): pp. 1611-1624, 2015 
[75] R. Speicher. Free probability theory. The Oxford handbook of random matrix theory, pp. 452-470, Oxford University Press, Oxford, 2011.

[76] J. L. Taylor. A general framework for a multi-operator functional calculus. Advances in Mathematics 9: pp. 183-252, 1972.

[77] J. L. Taylor. Functions of several noncommuting variables. Bulletin of the American Mathematical Society 79(1): pp. 1-34, 1973.

[78] D. V. Voiculescu. Free analysis questions I: Duality transform for the coalgebra of $\partial_{X: B}$. International Mathematics Research Notices 16: pp. 793-822, 2004.

[79] D. V. Voiculescu. Free analysis questions II: The Grassmannian completion and the series expansion at the origin. Journal für die reine und angewandte Mathematik (Crelles Journal) 645: pp. 155-236, 2010.

[80] D. V. Voiculescu, K. J. Dykema and A. Nica. Free random variables. A noncommutative probability approach to free products with applications to random matrices, operator algebras and harmonic analysis on free groups. CRM Monograph Series 1, American Mathematical Society, Providence, RI, 1992.

[81] J. Volčič. On domains of noncommutative rational functions. Linear Algebra and its Applications 516: pp. 69-81, 2017.

[82] J. Volčič. Matrix coefficient realization theory of noncommutative rational functions. Journal of Algebra 499: pp. 397-437, 2018.

(MP) Department of Mathematics, Ben-Gurion University of the Negev, P.O. Box 653, BeER-SheVa 84105, ISRAel

Email address: motpor@gmail.com

(VV) Department of Mathematics, Ben-Gurion University of the Negev, P.O. Box 653, Beer-Sheva 84105, Israel

Email address: vinnikov@math.bgu.ac.il 